ORNL/TM-2003/114

\title{
EFFECTS OF TEMPERATURE AND ENVIRONMENT ON MECHANICAL PROPERTIES OF TWO CHOPPED-FIBER AUTOMOTIVE STRUCTURAL COMPOSITES
}

M. B. Ruggles-Wrenn 


\section{DOCUMENT AVAILABILITY}

Reports produced after January 1, 1996, are generally available free via the U.S. Department of Energy (DOE) Information Bridge.

\section{Web site http://www.osti.gov/bridge}

Reports produced before January 1, 1996, may be purchased by members of the public from the following source.

National Technical Information Service

5285 Port Royal Road

Springfield, VA 22161

Telephone 703-605-6000 (1-800-553-6847)

TDD 703-487-4639

Fax 703-605-6900

E-mail info@ntis.fedworld.gov

Web site http://www.ntis.gov/support/ordernowabout.htm

Reports are available to DOE employees, DOE contractors, Energy Technology Data Exchange (ETDE) representatives, and International Nuclear Information System (INIS) representatives from the following source.

Office of Scientific and Technical Information

P.O. Box 62

Oak Ridge, TN 37831

Telephone 865-576-8401

Fax 865-576-5728

E-mail reports@adonis.osti.gov

Web site http://www.osti.gov/contact.html

This report was prepared as an account of work sponsored by an agency of the United States Government. Neither the United States Government nor any agency thereof, nor any of their employees, makes any warranty, express or implied, or assumes any legal liability or responsibility for the accuracy, completeness, or usefulness of any information, apparatus, product, or process disclosed, or represents that its use would not infringe privately owned rights. Reference herein to any specific commercial product, process, or service by trade name, trademark, manufacturer, or otherwise, does not necessarily constitute or imply its endorsement, recommendation, or favoring by the United States Government or any agency thereof. The views and opinions of authors expressed herein do not necessarily state or reflect those of the United States Government or any agency thereof. 
Metals and Ceramics Division

\section{EFFECTS OF TEMPERATURE AND ENVIRONMENT ON MECHANICAL PROPERTIES OF}

TWO CHOPPED-FIBER AUTOMOTIVE STRUCTURAL COMPOSITES

M. B. Ruggles-Wrenn

Date Published: 2003

Prepared by the OAK RIDGE NATIONAL LABORATORY

Oak Ridge, Tennessee 37831-6285 managed by

UT-BATTELLE, LLC

for the

U.S. DEPARTMENT OF ENERGY

under contract DE-AC05-00OR22725 
This page left blank intentionally. 


\section{CONTENTS}

Page

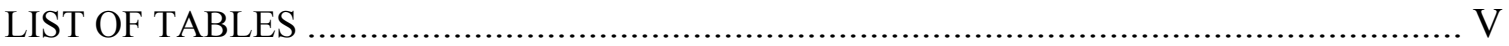

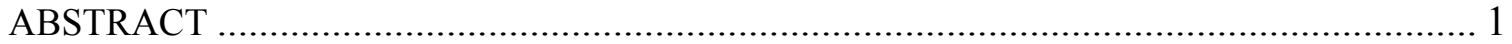

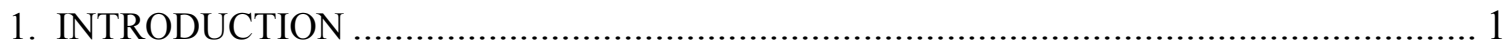

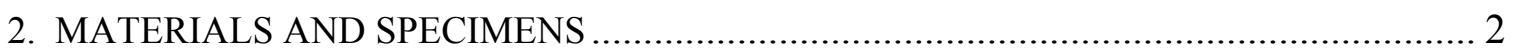

3. CHOPPED GLASS-FIBER COMPOSITE ................................................................. 3

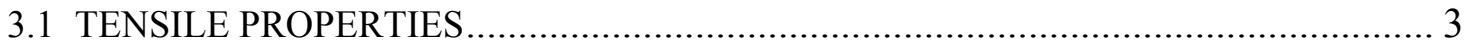

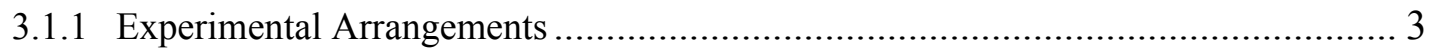

3.1.2 Room-Temperature Behavior .................................................................... 3

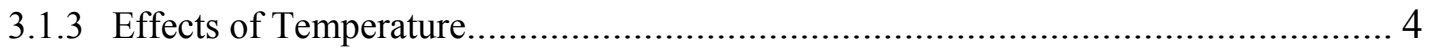

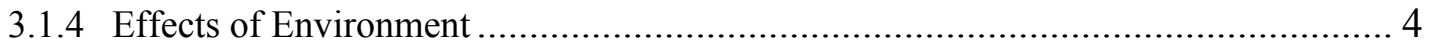

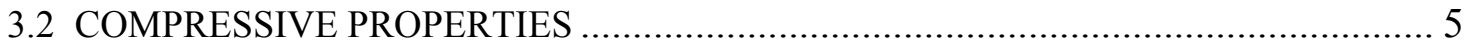

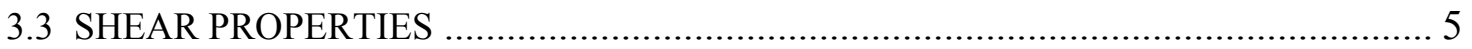

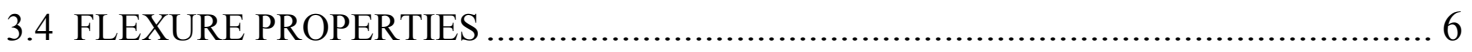

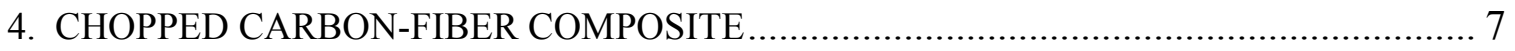

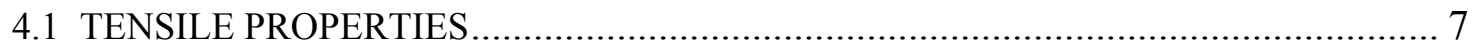

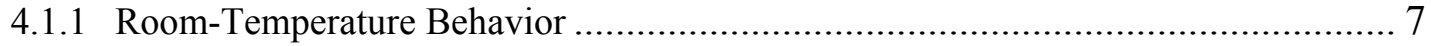

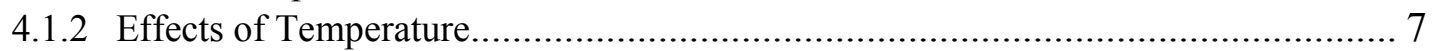

4.1.3 Effects of Environment .......................................................................... 8

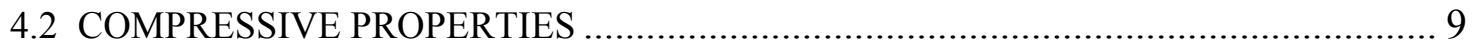

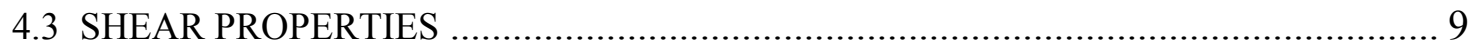

4.4 UNIAXIAL AND BIAXIAL FLEXURAL PROPERTIES .......................................... 10

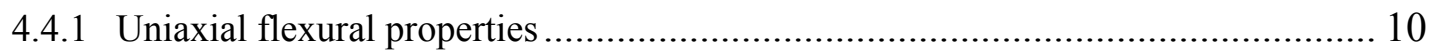

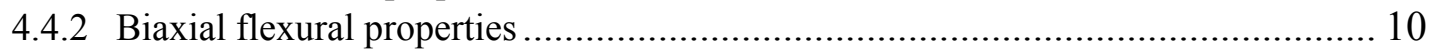

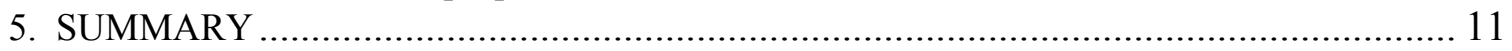

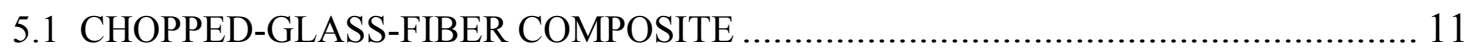

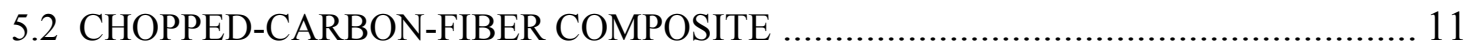

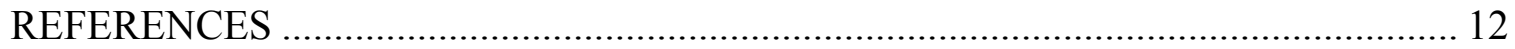


This page left blank intentionally. 


\section{LIST OF TABLES}

Table

1. Summary of room-temperature stiffness values for the chopped glass-fiber composite

2. Summary of room-temperature tensile test results for the chopped glass-fiber composite

3. Summary of plaque average tensile properties for the chopped glass-fiber composite

4. Tensile properties of the chopped glass-fiber composite at different temperatures......22

5. Effect of exposure in $50^{\circ} \mathrm{C}$ distilled water on tensile strength and stiffness of the chopped glass-fiber composite in the

6. Effect of exposure in $50^{\circ} \mathrm{C}$ distilled water on tensile strength and stiffness of the chopped glass-fiber composite pre-dried for $912 \mathrm{~h}$ in vacuum at $120^{\circ} \mathrm{C}$ 26

7. Effect of $2160-\mathrm{h}$ exposure in $50^{\circ} \mathrm{C}$ distilled water on tensile strength and stiffness of the chopped glass-fiber composite at $50^{\circ} \mathrm{C}$

8. Effect of 1008 -h exposure in $36^{\circ} \mathrm{C}$ distilled water on room-temperature tensile strength and stiffness of the chopped glass-fiber composite

9. Effect of exposure in $23^{\circ} \mathrm{C}$ distilled water on room-temperature tensile strength and stiffness of the chopped glass-fiber composite

10. Effect of exposure in windshield washer fluid on tensile strength and stiffness of the chopped glass-fiber composite

11. Summary of in-air room-temperature compression tests for the chopped glassfiber composite

12. Compressive properties of the chopped glass-fiber composite at different temperatures

13. Effects of exposure in $50^{\circ} \mathrm{C}$ distilled water on room-temperature compressive properties of the chopped glass-fiber composite

14. Summary of in-air room-temperature shear tests for the chopped glass-fiber composite

15. Shear properties of the chopped glass-fiber composite at different temperatures .........33

16. Effects of environment on room-temperature shear properties of the chopped glass-fiber composite

17. Summary of in-air room-temperature flexure tests for the chopped glass-fiber composite

18. Flexural properties of the chopped glass-fiber composite at different temperatures

19. Effects of environment on flexural properties of the chopped glass-fiber composite

20. Summary of room-temperature stiffness values for the 3-mm-thick chopped carbon-fiber composite

21. Summary of room-temperature stiffness values for the $1.5-\mathrm{mm}$-thick chopped carbon-fiber composite.

22. Summary of room-temperature tensile properties for the 3-mm-thick chopped carbon-fiber composite

23. Summary of room-temperature tensile properties for the $1.5-\mathrm{mm}$-thick chopped carbon-fiber composite

24. Summary of plaque average tensile properties the 3-mm-thick chopped-carbonfiber composite 
25. Summary of plaque average tensile properties for the $1.5-\mathrm{mm}$-thick choppedcarbon-fiber composite.

26. Summary of tensile results for specimens of various widths for the 3-mm-thick chopped-carbon-fiber composite

27. Summary of tensile tests at various strain rates for the

28. Summary of tensile tests at various temperatures for the 3-mm-thick chopped carbon-fiber composite

29. Temperature multiplication factors for determining at-temperature tensile modulus and strength from room-temperature values for the 3-mm-thick chopped-carbon-fiber composite

30. Summary of Poisson's ratio measurements at different temperatures for the 3mm-thick chopped carbon-fiber composite.

31. Effects of prior thermal cycling on mechanical properties of the 3-mm-thick chopped carbon-fiber composite

32. Effects of prior cooling to $-40^{\circ} \mathrm{C}$ on tensile properties of the 3-mm-thick chopped carbon-fiber composite

33. Effect of exposure in $23^{\circ} \mathrm{C}$ distilled water on tensile strength and stiffness of the 3-mm-thick chopped-carbon-fiber composite

34. Effect of exposure in $\mathbf{7 0 \%}$ relative humidity air on tensile strength and stiffness of 3-mm-thick chopped-carbon-fiber composite.

35. Effect of 1000-h exposure in $23^{\circ} \mathrm{C}$ distilled water on tensile strength and stiffness of 1.5-mm-thick chopped-carbon-fiber composite

36. Effects of 100-h exposure in windshield washer fluid on tensile stiffness and strength of 3-mm-thick chopped-carbon-fiber composite.

37. Summary of room-temperature compressive tests for the 3-mm-thick choppedcarbon-fiber composite

38. Summary of in-air room-temperature compressive properties of the $1.5-\mathrm{mm}$ thick chopped-carbon-fiber composite

39. Compressive properties of the 3-mm-thick chopped-carbon-fiber composite at different temperatures.

40. Temperature multiplication factors for determining at-temperature compressive stiffness and strength from room-temperature values for the 3-mm-thick chopped-carbon-fiber composite

41. Effects of $1000-h$ exposure in $23^{\circ} \mathrm{C}$ distilled water on room-temperature compressive properties of the 3-mm-thick chopped-carbon-fiber composite

42. Effects of $1000-h$ exposure in $23^{\circ} \mathrm{C}$ distilled water on room-temperature compressive properties of the 1.5-mm-thick chopped-carbon-fiber composite

43. Effects of exposure in windshield washer fluid on room-temperature compressive strength of the 3-mm-thick chopped-carbon-fiber composite

44. Summary of in-air room-temperature shear properties for

45. Summary of in-air room-temperature shear properties for the $1.5-\mathrm{mm}$-thick chopped-carbon-fiber composite.

46. Shear properties of the 3-mm-thick chopped-carbon-fiber composite at different temperatures

47. Temperature multiplication factors for determining at-temperature shear modulus and strength from room-temperature values for the 3-mm-thick chopped-carbon-fiber composite.

48. Effects of 1000 -h exposure in $23^{\circ} \mathrm{C}$ distilled water on room-temperature shear properties of the 3-mm-thick chopped-carbon-fiber composite

49. Effects of exposure $1000-\mathrm{h}$ in $23^{\circ} \mathrm{C}$ distilled water on room-temperature shear properties of the 1.5-mm-thick chopped-carbon-fiber composite 
50. Effects of 100-h exposure in windshield washer fluid on room-temperature shear strength of the 3-mm-thick chopped-carbon-fiber composite

51. Room-temperature in-air uniaxial flexural properties of the 3-mm-thick chopped-carbon-fiber composite

52. Room-temperature in-air uniaxial flexural properties of the 1.5 -mm-thick chopped-carbon-fiber composite

53. Uniaxial flexural properties of the 3-mm-thick chopped-carbon-fiber composite at different temperatures

54. Effects of 1000-h exposure in $23^{\circ} \mathrm{C}$ distilled water on uniaxial flexural properties of 3-mm-thick chopped-carbon-fiber composite.

55. Effects of 100-h exposure in windshield washer fluid on uniaxial flexural properties of 3-mm-thick chopped-carbon-fiber composite

56. Biaxial flexural properties of the 3-mm-thick chopped-carbon-fiber composite at different temperatures.

57. Biaxial flexural properties of the 1.5-mm-thick chopped-carbon-fiber composite at different temperatures .

58. Effects of environment on biaxial flexural properties of the 3-mm-thick choppedcarbon-fiber composite. 
This page left blank intentionally. 


\title{
EFFECTS OF TEMPERATURE AND ENVIRONMENT ON MECHANICAL PROPERTIES OF TWO CHOPPED-FIBER AUTOMOTIVE STRUCTURAL COMPOSITES
}

\author{
M. B. Ruggles-Wrenn
}

\begin{abstract}
The Durability of Lightweight Composite Structures Project was established at Oak Ridge National Laboratory (ORNL) by the U.S. Department of Energy to provide the experimentally-based, durability-driven design guidelines necessary to assure long-term structural integrity of automotive composite components. The initial focus of the ORNL Durability Project was on composite materials consisting of polyurethane reinforced with E-glass. Current focus of the project is on composite materials reinforced with carbon fibers. The primary purpose of this report is to provide the individual specimen test date. Basic mechanical property testing and results for two chopped-fiber composite materials, one reinforced with glass- and the other with carbon fiber are provided. Both materials use the same polyurethane matrix. Preforms for both materials were produced using the $\mathrm{P} 4$ process. Behavioral trends, effects of temperature and environment, and corresponding design knockdown factors are established for both materials. Effects of prior short-time loads and of prior thermal cycling are discussed.
\end{abstract}

\section{INTRODUCTION}

Development of lighter weight, more fuel-efficient automobiles represents a technology area where advanced materials can be successfully applied. The fuel efficiency of automobiles can be significantly enhanced by using lightweight materials, such as polymer matrix composites, in primary structural components. However, while significant effort is being devoted to material development and processing, commercial application of composite materials lags behind due, in part, to the lack of specific design guidance and an understanding of the material performance under actual service conditions. There is a recognized need for improved structural design methods and criteria that address deformation and failure behavior of composite materials.

The Durability of Lightweight Composite Structures Project was established at Oak Ridge National Laboratory (ORNL) by the U.S. Department of Energy to provide the experimentally-based, durability-driven design guidelines necessary to assure long-term structural integrity of automotive composite components. The initial ORNL Durability Project focused on characterizing and understanding the mechanical behavior of candidate random-glass-fiber automotive composites. The focus of the current companion project is reflected in the name Durability of Carbon-Fiber Composites. The present report describes basic tensile, compressive, shear, and flexure property testing and results for two chopped-fiber composites produced by a rapid molding process suitable for high-volume automotive applications. The two composites had the same Baydur 420 IMR urethane matrix reinforced with random chopped-glass-fiber in the case of one composite and chopped-carbon-fiber in the case of the other. The preforms for both materials were produced using the $\mathrm{P} 4$ process.

Materials and specimen designs are presented in Chapter 2. Chapter 3 is dedicated to behavior of the glass-fiber composite. Experimental arrangements are described and tensile, compressive and shear test results are summarized. Effects of temperature and environment are assessed, corresponding design knockdown factors are established. In addition, effects of prior short-time tensile loads and prior thermal cycling are discussed. Chapter 4 focuses on the carbonfiber composite, giving a summary of tests and test results, as well as design knockdown factors to account for the effects of temperature and environment. Concluding remarks are made in Chapter 5. 


\section{MATERIALS AND SPECIMENS}

The matrix for both materials used in this study was produced by the Bayer Corporation and identified as Baydur 420 IMR (Internal Mold Release). Preforms for both composites were produced using the $\mathrm{P} 4$ process developed by Owens-Corning in Battice, Belgium. In these preforms, chopped glass fibers were used in the case of one material, and chopped carbon fiber in the case of the other. Both materials were provided by the Automotive Composites Consortium (ACC). Chopped-glass-fiber material plaques were nominally $3.2 \mathrm{~mm}$ thick. In the case of the chopped-carbon-fiber composite, two classes of plaques were characterized. One class, denoted $\mathrm{Z}$, had a nominal thickness of $3.0 \mathrm{~mm}$, the other, denoted $\mathrm{ZZ}$, a nominal thickness of $1.5 \mathrm{~mm}$.

Specimens were cut from plaques in a single direction. In the case of the chopped-glass-fiber (B) composite, most of the specimens used in tensile and stiffness tests were flat, untabbed, and dogboned. However, in some cases straight-sided specimens with tabs were used. Flat specimens with tabs were used in compression tests. The V-notched beam (Iosipescu) shear specimens with tabs were used in shear tests. The tab material was G-11 composite, an epoxy reinforced with woven fiberglass cloth. The adhesive used for bonding the tabs to the test specimens was Hysol EA $93009 \mathrm{NA} \mathrm{A} / \mathrm{B}$. An experimental version of this adhesive was successfully used for $120^{\circ} \mathrm{C}$ testing. The dogbone tensile and the Iosipescu shear specimens were as described in Ref. 4. Beam specimens used in uniaxial flexure tests were $25.4 \mathrm{~mm} \times 75 \mathrm{~mm} \times 3.2 \mathrm{~mm}$. For the tabbed specimens dimensions were as follows:

Tension

Specimen: $\quad 25.4 \mathrm{~mm} \times 3.2 \mathrm{~mm} \times 203 \mathrm{~mm}$

Tabs: $\quad 25.4 \mathrm{~mm} \times 3.2 \mathrm{~mm} \times 50.8 \mathrm{~mm}$

\section{Compression}

$25.4 \mathrm{~mm} \times 3.2 \mathrm{~mm} \times 133 \mathrm{~mm}$ $25.4 \mathrm{~mm} \times 1.6 \mathrm{~mm} \times 57.2 \mathrm{~mm}$

For the chopped-carbon-fiber composite, specimens used in stiffness and tensile tests were untabbed and dogbone-shaped in the case of the 3-mm-thick material, and straight-sided with tabs in the case of the 1.5-mm-thick material. Straight-sided specimens with tabs were used in compression tests. Specimen and tab dimensions were as specified above, with the exception of the compression specimen length, which was $127 \mathrm{~mm}$. The same tab material was used as in the case of the chopped-glass-fiber composite. The V-notched Iosipescu shear specimens with tabs were as described in Ref. 4. Tabs were cut from the 3-mm-thick chopped-carbon-fiber composite. The high-temperature adhesive used for bonding the tabs to the test specimens was Hysol EA $9394 \mathrm{~A} / \mathrm{B}$ an outside diameter of $94 \mathrm{~mm}$ were used in biaxial flexure tests. 


\section{CHOPPED GLASS-FIBER COMPOSITE}

\subsection{TENSILE PROPERTIES}

\subsubsection{Experimental Arrangements}

All tests were performed in an air environment. A servocontrolled MTS axial-torsion mechanical testing machine together with an MTS digital TestStar Materials Testing Workstation was used for computerized testing and data acquisition. The load (engineering stress), strain, and displacement were measured and recorded; the digitized test data were stored on both hard and floppy disks. The data acquisition intervals were established on the basis of load, i.e. a data point was recorded whenever the load changed by $44.5 \mathrm{~N}$. After the test the digitized data can be recalled for processing and interpretation. The entire history is available for analysis.

Specimens were mounted in mechanical wedge grips. In the case of dogbone-shaped and straight-sided specimens, strain measurement was accomplished with an MTS 632.17E-20 averaging extensometer of 25.4-mm gage length. Elastic modulus, E, was measured in three loadcontrolled cycles between load levels of 445 (corresponding to $\approx 4 \%$ of the UTS) and $2224 \mathrm{~N}$ (corresponding to $\approx 20 \%$ of the UTS) with a frequency of $0.1 \mathrm{~Hz}$. The recorded elastic modulus was established as the average value obtained during cycling. Tensile tests to failure were conducted in displacement control at a rate of $0.025 \mathrm{~mm} / \mathrm{s}$.

\subsubsection{Room-Temperature Behavior}

Results of stiffness and tensile tests are summarized in Tables 1 and 2. Values of elastic modulus, E, obtained in 934 stiffness tests are presented in Table 1. The ultimate tensile strength, UTS, failure strain, $\varepsilon_{\mathrm{f}}$, and proportional limit stress and strain produced in 94 tensile tests to failure are summarized in Table 2. Also given in Tables 1 and 2 are the overall average, A, standard deviation, $\mathrm{S}$, and coefficient of variation, $\mathrm{COV}$, for each of the properties determined. The coefficient of variation is defined as $\mathrm{COV}=(\mathrm{S} / \mathrm{A}) \times 100 \%$ and gives a measure of the scatter of a particular data set. Proportional limit was defined as the point $\left(\sigma_{\mathrm{PL}}, \varepsilon_{\mathrm{PL}}\right)$ in stress-strain space where the tensile stress-strain curve departs from linearity. The following procedure was adopted to calculate proportional limit stress and strain values:

(i) Establish the elastic stiffness value from an initial stiffness test.

(ii) Calculate "running" stiffness for each pair of the stress-strain values forming the remaining stress-strain curve.

(iii) Divide the "running" stiffness by the elastic stiffness. The point in stress-strain space where the stiffness ratio becomes, and remains, less than 1.0 is the proportional limit.

Specimen numbers contain reference to the plaque number. For example, number B11-1 refers to the tensile specimen 1 from plaque B11. In the case of plaque B9, straight-sided tabbed specimens were used to measure stiffness in two locations along the axis of the specimen by positioning the extensometer directly above and directly below the center of the specimen. Thus number B9-19-1 corresponds to the stiffness measured above the center of specimen 19 cut from plaque 9, and number B9-19-2 refers to the stiffness measured below the center of the same specimen. In the case of plaques B18-B21, numbers identify reference tensile specimens cut from those plaques. For example, number B18-R3 refers to the reference tensile specimen R3 cut from plaque B18. Finally, in the case of plaques B22-B30, tensile tests were conducted on straightsided tabbed specimens cut from an undamaged portion of impact specimens (see cutting plan in 
Ref. 2). Specimen number B22-1-4 refers to the tensile specimen 4 cut from impact specimen 1 from plaque B22.

The coefficients of variation (COV) are given in Tables 1 and 2 to provide some measure of the data scatter. It is seen that stiffness values exhibit the least amount of scatter (COV $=8.49 \%)$, followed by UTS $(\mathrm{COV}=10.4 \%)$ and failure strain $(\mathrm{COV}=10.5)$. Average results indicate that the proportional limit stress is $19 \%$ of the UTS, and the proportional limit strain is $15 \%$ of the failure strain. It is also noteworthy that no significant influence of the specimen shape on stiffness or tensile properties is observed.

Stiffness and tensile test results are recapitulated in Table 3, where the number of stiffness and tensile tests conducted on specimens from each particular plaque and the plaque averages of the tensile properties E, UTS, and $\varepsilon_{\mathrm{f}}$, are given. Plaques are referred to using both the ORNL and the ACC nomenclature. Table 3 permits an easy assessment of plaque-to-plaque variations in tensile properties, "strong" and "weak" plaques can be readily identified. Overall averages of the tensile properties are included at the bottom of Table 3 for an easy comparison of the individual plaque behavior with that of the rest of the population.

\subsubsection{Effects of Temperature}

The effect of temperature on tensile properties was assessed based in 45 tensile tests conducted at $-40^{\circ}, 23^{\circ}, 50^{\circ}$, and $120^{\circ} \mathrm{C}$. The test procedure was as specified in Ref. 3. Specimens from two plaques (B4 and B7) were used. Test results are summarized in Table 4. A temperature multiplication factor for determining stiffness and strength from room-temperature values was developed based on the test results in Table 4. Derivation of the multiplication factor is discussed in detail in Ref. 1.

\subsubsection{Effects of Environment}

Effects of moisture on tensile properties were investigated. A broad range of exposure conditions and exposure times of up to $6168 \mathrm{~h}$ were employed to provide a better understanding of the moisture absorption process. Two groups of specimens were exposed to $50^{\circ} \mathrm{C}$ distilled water. The first group was exposed in the "as received" condition. The second group was dried for $915 \mathrm{~h}$ in vacuum at $120^{\circ}$, resulting in an average weight loss of $0.41 \%$. Effects of exposure in $50^{\circ} \mathrm{C}$ distilled water on tensile properties are summarized in Tables 5 and 6 for the "as-received" and the pre-dried specimens, respectively. In addition, six specimens in the "as-received" condition were immersed in $50^{\circ} \mathrm{C}$ distilled water for $2160 \mathrm{~h}$ and then tested at $50^{\circ} \mathrm{C}$. Results are summarized in Table 7. Furthermore, to assess the effect of temperature on environmental degradation, six specimens in the "as-received" condition were immersed for $1008 \mathrm{~h}$ in $36^{\circ} \mathrm{C}$ distilled water and then tested at $23^{\circ} \mathrm{C}$. Results are presented in Table 8. Finally, the effects of exposure in $23^{\circ} \mathrm{C}$ distilled water were investigated. Exposure times were $100 \mathrm{~h}$ (six specimens) and $1000 \mathrm{~h}$ (six specimens). Results are shown in Table 9. Included in Tables 5-9 are the virgin stiffness, post-exposure stiffness and strength, as well as the values of percent change in strength and stiffness for given exposure times. A detailed discussion of moisture sorption and correlations between exposure time, weight change, and property changes can be found in Ref. 1 .

The effects of exposure in windshield washer fluid (30\% distilled water and $70 \%$ methanol) were investigated. Exposure times were 100, 1000, and $2160 \mathrm{~h}$. Test results are summarized in Table 10. The summary in Table 10 includes virgin stiffness (i. e. stiffness prior to exposure), as well as post-exposure stiffness, UTS and failure strain for each specimen. Also included in Table 10 are environmental knockdown factors for design. The stiffness design factor is defined as a ratio of post-exposure stiffness to virgin stiffness of that particular specimen. Strength design factor is calculated as a ratio of the post-exposure UTS to the average UTS obtained in tensile tests on unexposed specimens from the same plaque. Finally an overall stiffness (strength) design 
factor for a given exposure condition is established as an average of stiffness (strength) design factors for that condition.

\subsection{COMPRESSIVE PROPERTIES}

In-air room-temperature compressive properties were established based on 17 tests on specimens from plaques B16 and B17. Compression tests employed an IITRI fixture (Procedure B in ASTM D 3410 3 ). The test method was as described in Ref. 3. Test results are summarized in Table 11, where compressive stiffness, $\mathrm{E}_{\mathrm{C}}$, is given together with ultimate compressive strength (UCS) and failure strain.

The effect of temperature on compressive properties was investigated in 39 tests: 17 tests at $23^{\circ} \mathrm{C}$ (see Table 11), seven tests each at $50^{\circ}$ and $120^{\circ} \mathrm{C}$, and 8 tests at $-40^{\circ} \mathrm{C}$. The test procedure was as specified in Ref. 3. Test results obtained at $50^{\circ}, 120^{\circ}$, and $-40^{\circ} \mathrm{C}$ are summarized in Table 12. Temperature multiplication factors for determining compressive stiffness and strength from room-temperature values were developed based on the test results in Tables 11 and 12. Derivation of the design factors is discussed in details in Ref. 1. Temperature multiplication factors for determining compressive stiffness and strength from room-temperature values were developed based on the test results in Tables 11 and 12. Derivation of the design factors is discussed in details in Ref. 1.

Effects of environment on compressive properties were assessed in 31 tests on specimens presoaked in $50^{\circ} \mathrm{C}$ distilled water. Twelve specimens were exposed for $912 \mathrm{~h}$, and nineteen specimens, for $2160 \mathrm{~h}$. Results are summarized in Table 13. The summary in Table 13 includes compressive stiffness, $\mathrm{E}_{\mathrm{C}}$, UCS and failure strain for each specimen. Also included in Table 13 are environmental knockdown factors for design. The stiffness (strength) design factor is defined as the ratio of post-exposure stiffness (strength) to average stiffness (strength) obtained in compression tests on unexposed specimens from the same plaque.

\subsection{SHEAR PROPERTIES}

In-air room-temperature shear properties were determined in tests on 11 Iosipescu specimens from 3 different plaques (B19-B21). The test method was as described in Ref. 3. Test results are summarized in Table 14, where elastic shear modulus, G, shear strength, and failure strain are presented.

The effect of temperature on shear properties was investigated in 25 Iosipescu shear tests: 11 tests at $23^{\circ} \mathrm{C}$ (see Table 14 ), 6 tests at $50^{\circ} \mathrm{C}$, and 8 tests at $120^{\circ} \mathrm{C}$. Test procedure was as specified in Ref. 3. Test results obtained at $50^{\circ}$ and $120^{\circ} \mathrm{C}$ are summarized in Table 15 . Temperature multiplication factors for determining shear modulus and strength from room-temperature values were developed based on the test results in Tables 14 and 15. Derivation of the design factors is discussed in details in Ref. 1.

Two fluids, $50^{\circ} \mathrm{C}$ distilled water and windshield washer fluid, were used to assess environmental degradation of shear properties. Exposure times were $2160 \mathrm{~h}$ and $100 \mathrm{~h}$ for the $50^{\circ} \mathrm{C}$ distilled water and windshield washer fluid, respectively. For specimens exposed in $50^{\circ} \mathrm{C}$ distilled water, the summary in Table 16 includes shear modulus, G, shear strength and failure strain. In the case of windshield washer fluid, shear modulus values were not measured. Application of strain gages, required for shear specimens, would have resulted in excessive loss of the absorbed methanol due to evaporation during gage installation. Also included in Table 16 are environmental knockdown factors for design. The stiffness (strength) design factor is defined as the ratio of post-exposure stiffness (strength) to the average stiffness (strength) obtained in shear tests on unexposed specimens (see Table 14). 


\subsection{FLEXURE PROPERTIES}

In-air room-temperature out-of-plane flexural stiffness, modulus of rupture (MOR), which is equal to the elastically calculated maximum bending stress at failure, and failure strain in flexure were determined in three-point bend tests. Dimensions of the beam specimen were $25.4 \mathrm{~mm} \times 75$ $\mathrm{mm} \times 3.2 \mathrm{~mm}$. The support span was $50 \mathrm{~mm}$. The test procedure, which employed an MTS 642.10 bend fixture, is described in Ref. 3. Six beam specimens from three different plaques (B18-B20) were tested at $23^{\circ} \mathrm{C}$. Results are summarized in Table 17.

The effect of temperature on flexure properties was investigated in 18 flexure tests, six tests at $23^{\circ} \mathrm{C}$ (see Table 17) and six tests each at $50^{\circ}$ and $120^{\circ} \mathrm{C}$. The test procedure was as specified in Ref. 3. Test results obtained at $50^{\circ}$ and $120^{\circ} \mathrm{C}$ are summarized in Table 18 , where flexural stiffness, MOR, and failure strain are given together with the stiffness and MOR design factors. Note that tests at $120^{\circ} \mathrm{C}$ did not include strain measurement.

Two fluids, distilled water and windshield washer fluid, were used to assess environmental degradation of flexure properties. Fourteen specimens were exposed in $50^{\circ} \mathrm{C}$ distilled water for $2160 \mathrm{~h}$. Six beam specimens were presoaked in $23^{\circ} \mathrm{C}$ distilled water for $1000 \mathrm{~h}$. Eight beam specimens were exposed in windshield washer fluid for $100 \mathrm{~h}$. All specimens were tested at $23^{\circ} \mathrm{C}$. Test results are presented in Table 19, where the values of MOR are given together with the MOR design knockdown factors. The MOR design factor is defined as the ratio of post-exposure MOR to the average MOR obtained in flexure tests on unexposed specimens (see Table 17). 


\section{CHOPPED CARBON-FIBER COMPOSITE}

\subsection{TENSILE PROPERTIES}

\subsubsection{Room-Temperature Behavior}

Results of stiffness tests are summarized in Tables 20 and 21 for the 3.0-mm thick and for the $1.5-\mathrm{mm}$ thick materials, respectively. Note that in the case of the $3.0-\mathrm{mm}$ thick material, standard dogbone-shaped specimens were used and a single stiffness measurement in the gage section of the specimen was made. The total number of stiffness tests for the 3-mm thick composite was 551. In the case of the 1.5-mm thick material, straight-sided specimens with tabs were employed. Furthermore, stiffness was measured at 3 locations along the axis of the specimen: (1) above the central 25.4-mm gage section $\left(E_{1}\right),(2)$ in the gage section $\left(E_{2}\right)$, and (3) below the gage section $\left(E_{3}\right)$. The total number of stiffness tests for the $1.5-\mathrm{mm}$ thick composite was 367 . An averaging extensometer of 25.4-mm gage length was used in all stiffness tests. Experimental arrangements were as described in Sect. 3.1.1. In addition to the values of elastic modulus, E, Tables 20 and 21 include corresponding averages, standard deviations, and coefficients of variation.

Results of 125 tensile tests for the 3-mm thick composite material are summarized in Table 22. Presented in Table 23 are results of 81 tensile tests for the $1.5-\mathrm{mm}$ thick composite. The ultimate tensile strength, UTS, and failure strain, $\varepsilon_{\mathrm{f}}$, are shown in Tables 22 and 23 together with the overall average, standard deviation, and coefficient of variation for each of the variables. In addition, failure location for each of the 1.5-mm-thick specimens is given in Table 23. Note that for each specimen the failure location in Table 23 corresponds to the lowest stiffness value measured for that particular specimen (see Table 21).

Stiffness and tensile test results are recapitulated in Tables 24 and 25, for the 3-mm thick ( $Z$ composite) and the $1.5-\mathrm{mm}$ thick (ZZ composite) materials, respectively. Shown in Tables 24 and 25 are the number of stiffness and tensile tests conducted on specimens from each particular plaque and the plaque averages of the tensile properties E, UTS, and $\varepsilon_{\mathrm{f}}$. Tables 24 and 25 permit an assessment of plaque-to-plaque variations in tensile properties as well as comparison of scatter within each individual plaque. Overall averages of the tensile properties are included at the bottom of Tables 24 and 25 .

A series of tensile tests on specimens of various widths was performed to assess whether the relatively narrow $20-\mathrm{mm}$-wide specimens gave representative tensile properties for the $3-\mathrm{mm}$ thick chopped carbon-fiber composite. Three sets of four tensile specimens having widths of 10.2 , 20.3, and $40.6 \mathrm{~mm}$ were cut from plaque $\mathrm{Z1}$ and tested. In addition, 12 tensile tests were performed on 73.5-mm-wide specimens cut from different plaques. Results are summarized in Table 26. Note that strain, and consequently stiffness, were not measured for the 73.5 -mm-wide specimens.

Results, presented in Table 26, demonstrate that both tensile stiffness and strength increase with specimen width. Specimens of 20.2- $\mathrm{mm}$ width were chosen for baseline tensile and fatigue tests with the knowledge that they would give relatively conservative stiffness and strength values while being sufficiently wide to be representative of the composite. A width factor was established to permit UTS adjustment for specimens of various widths (see Chap. 7 of Ref. 4).

The effect of strain rate on tensile behavior was investigated in four tests conducted at each of the following constant strain rates: $10^{-6}, 10^{-4}, 10^{-2}$, and $10 \mathrm{~s}^{-1}$. Results are summarized in Table 27.

\subsubsection{Effects of Temperature}

Effects of temperature on tensile properties of the 3-mm thick chopped carbon-fiber composite were assessed in six tests at each of the following temperatures: $-40^{\circ},-20^{\circ}, 0^{\circ}, 23^{\circ}$, $50^{\circ}, 70^{\circ}$, and $120^{\circ} \mathrm{C}$. All 42 tests in the temperature study were conducted on specimens from a 
single plaque Z20. Results are summarized in Table 28, where the virgin (room-temperature) stiffness is shown together with the at-temperature stiffness, UTS, and failure strain. Also given in Table 28 are stiffness and strength temperature factors for each specimen. The stiffness temperature factor is defined as the ratio of at-temperature stiffness for a particular specimen to the virgin room-temperature stiffness. The strength temperature factor is defined as the ratio of the at-temperature UTS for a particular specimen to the average UTS obtained at roomtemperature for specimens from the same plaque. Results in Table 28 were used to derive temperature multiplication factors to determine at-temperature stiffness and strength from roomtemperature values. The factors are presented in Table 29. The detailed derivation of the temperature multiplication factors can be found in Ref. 4.

Poisson's ratio measurements were carried out by subjecting six tensile specimens to 5 loadcontrolled cycles between 445 (corresponding to $\approx 3.5 \%$ of the room-temperature UTS) and 2224 $\mathrm{N}$ (corresponding to $\approx 18 \%$ of the room-temperature UTS) at a frequency of $0.1 \mathrm{~Hz}$. Each specimen was tested at $-40^{\circ}, 23^{\circ}, 70^{\circ}$, and $120^{\circ} \mathrm{C}$, and remained gripped in the test machine between temperature changes. Using low loads ensures that damage was not introduced at each temperature. Poisson's ratio values for the 3-mm-thick chopped carbon-fiber composite are summarized in Table 30.

The effects of prior thermal cycling on basic properties of the 3-mm thick chopped carbonfiber composite were investigated in six tensile, six compressive, and five shear tests. Prior to testing, specimens were subjected to 25 thermal cycles. A thermal cycle between $-40^{\circ}$ and $120^{\circ} \mathrm{C}$ (shown in Ref. 4) was chosen to reflect the automotive design temperature range. Results are summarized in Table 31. In addition, a group of six specimens was cooled down to $-40^{\circ} \mathrm{C}$, held at that temperature for $1 \mathrm{~h}$, then returned to $23^{\circ} \mathrm{C}$ and tested. The purpose of this exercise was to determine whether cooling to $-40^{\circ} \mathrm{C}$ had a permanent effect on strength and stiffness. Results, given in Table 32, demonstrate that prior cooling does not result in a permanent strength reduction. Once returned to $23^{\circ} \mathrm{C}$, the material regains its room-temperature strength. In fact, for both stiffness and strength, prior cooling appears to be slightly beneficial.

\subsubsection{Effects of Environment}

The effects of moisture on tensile properties of the 3-mm-thick chopped carbon-fiber composite were investigated with the purpose of establishing correlations between exposure time and weight change, and subsequently, strength and stiffness. One group of specimens was exposed in $23^{\circ} \mathrm{C}$ distilled water. Another group was exposed in $70 \%$ relative humidity $(\mathrm{RH})$ air. All specimens were kept in $40 \% \mathrm{RH}$ air for one week prior to exposure. The effects of exposure in $23^{\circ} \mathrm{C}$ distilled water and in $70 \% \mathrm{RH}$ air on tensile properties are summarized in Tables 33 and 34, respectively. Included in Tables 33 and 34 are the virgin stiffness, post-exposure stiffness and strength, as well as the values of percent change in strength and stiffness for given exposure times. The change in stiffness is calculated with reference to the virgin stiffness of each individual specimen. The change in strength is calculated with reference to the plaque average strength. A detailed discussion of moisture sorption and correlations between exposure time, weight change, and property changes can be found in Ref. 4.

The effects of $1000-\mathrm{h}$ exposure in $23^{\circ} \mathrm{C}$ distilled water on tensile properties of the $1.5-\mathrm{mm}-$ thick composite were investigated using 28 baseline and 28 pre-exposed specimens from plaque ZZ5. Results are summarized in Table 35. The effects of exposure in windshield washer fluid (30\% distilled water and $70 \%$ methanol) on tensile properties were investigated. Windshield washer fluid was selected as a practical exposure condition because methanol in windshield washer fluid is a "lighter molecule." Exposure time was $100 \mathrm{~h}$. Test results are summarized in Table 36, where virgin stiffness, post-exposure stiffness, UTS and failure strain are given for each specimen. Also included in Table 36 are environmental knockdown factors for design. The stiffness design factor is defined as the ratio of post-exposure stiffness to virgin stiffness of that 
particular specimen. The strength design factor is calculated as the ratio of the post-exposure UTS to the average UTS obtained in tensile tests on unexposed specimens from the same plaque.

\subsection{COMPRESSIVE PROPERTIES}

All tests were performed in an air environment. Specimens were mounted in an IITRI Compression Test Fixture according to the ASTM Standard D $3410^{3}$. Compressive tests to failure were conducted in displacement control at a rate of $0.025 \mathrm{~mm} / \mathrm{s}$. Strain measurement was accomplished with two strain gages mounted in the gage section of the specimen, one strain gage on each side of the specimen. In most tests the two strain gages produced strain measurements that were approximately the same. The two strain measurements were averaged and the average strains were used for reporting data. The data acquisition intervals were established on the basis of load, i.e. a data point was recorded whenever the load changed by $44.5 \mathrm{~N}$. Compressive stiffness, $E_{C}$, was calculated using linear regression for the initial stress-strain slope between loads of 445 and $2224 \mathrm{~N}$ in the case of the 3-mm-thick material and between the loads of 223 and $1112 \mathrm{~N}$ in the case of the 1.5 -mm-thick composite.

In the case of the 3-mm-thick composite, 35 specimens from 11 different plaques were tested. For the 1.5-mm-thick composite, 8 specimens from plaque ZZ3 were used. Test results are summarized in Tables 37 and 38 for the $3-\mathrm{mm}$-thick and the 1.5 -mm-thick materials, respectively.

The effect of temperature on compressive properties of the 3-mm-thick chopped-carbon-fiber composite was investigated in 18 tests on specimens from plaque Z28: six tests at $-40^{\circ}, 70^{\circ}$ and $120^{\circ} \mathrm{C}$ each. Test results are summarized in Table 39. Results in Table 39 were used to derive temperature multiplication factors to determine at-temperature compressive stiffness and strength from room-temperature values (See Ref 4 ). The factors are presented in Table 40.

Effects of 1000-h exposure in $23^{\circ} \mathrm{C}$ distilled water on compressive properties of the $3-\mathrm{mm}$ thick composite were investigated using six baseline and five pre-exposed specimens from plaque Z28. Results for pre-exposed specimens are summarized in Table 41, where compressive stiffness, UCS and failure strain are given together with knockdown design factors for each specimen. The stiffness (strength) design factor is defined as the ratio of post-exposure stiffness (strength) to average stiffness (strength) obtained in compression tests on unexposed specimens from the same plaque. Effects of 1000 -h exposure in $23^{\circ} \mathrm{C}$ distilled water on compressive properties of the 1.5 -mm-thick composite were investigated using eight baseline and seven preexposed specimens from plaque ZZ3. Results are summarized in Table 42 for the pre-exposed specimens. Effects of 100-h exposure windshield washer fluid on compressive strength of the 3mm-thick composite were investigated using six baseline and five pre-exposed specimens from plaque Z28. Results for the pre-exposed specimens are summarized in Table 43. Note that in the case of windshield washer fluid, compressive stiffness values were not measured. Application of strain gages, required for compressive specimens, would have resulted in excessive loss of the absorbed methanol due to evaporation during gage installation.

\subsection{SHEAR PROPERTIES}

In-air room-temperature shear properties of the 3-mm-thick chopped-carbon-fiber composite were established based on 46 Iosipescu shear tests on specimens from 10 different plaques. In the case of the 1.5-mm-thick chopped-carbon-fiber composite, eight Iosipescu shear specimens from plaque ZZ3 were tested. Test results are summarized in Tables 44 and 45 for the 3-mm-thick and the 1.5 -mm-thick materials, respectively.

The effect of temperature on shear properties of the 3-mm-thick chopped-carbon-fiber composite was investigated in 16 tests on specimens from plaque Z28: five tests at $-40^{\circ}$ and $70^{\circ} \mathrm{C}$ each, and six tests at $120^{\circ} \mathrm{C}$. Test results are summarized in Table 46 . Results in Table 46 were 
used to derive temperature multiplication factors to determine at-temperature stiffness and strength from room-temperature values (See Ref 4). The factors are presented in Table 47.

Effects of $1000-\mathrm{h}$ exposure in $23^{\circ} \mathrm{C}$ distilled water on shear properties of the 3 -mm-thick composite were investigated using six baseline and five pre-exposed specimens from plaque Z28 and sixteen baseline and sixteen pre-exposed specimens from plaque Z9. In the case of the 1.5mm-thick material, eight baseline and eight pre-exposed specimens from plaque ZZ3 were employed. Shear modulus, shear strength, and failure strain for pre-exposed 3-mm and $1.5-\mathrm{mm}-$ thick specimens are given in Tables 48 and 49 together with stiffness and strength environmental design factors. The stiffness (strength) design factor is defined as the ratio of post-exposure stiffness (strength) to average stiffness (strength) obtained in shear tests on unexposed specimens from the same plaque.

Effects of 100-h exposure in windshield washer fluid on shear strength of the 3-mm-thick composite were investigated using six baseline and five pre-exposed specimens from plaque Z28. Results for the pre-exposed specimens are summarized in Table 50. Note that in the case of windshield washer fluid, shear stiffness values were not measured. Application of strain gages, required for compressive and shear specimens, would have resulted in excessive loss of the absorbed methanol due to evaporation during gage installation.

\subsection{UNIAXIAL AND BIAXIAL FLEXURAL PROPERTIES}

\subsubsection{Uniaxial flexural properties}

The uniaxial flexural strength tests were performed according to the three-point bend test method specified in ASTM Standard D 790. . The specimen width was $12.7 \mathrm{~mm}$, the support span was $50 \mathrm{~mm}$ (see Ref. 4), the loading rollers had a radius of $4.4 \mathrm{~mm}$. Eight uniaxial flexure tests were conducted at $23^{\circ} \mathrm{C}$ on specimens from plaque Z6. Results are presented in Table 51 in terms of the modulus of rupture (MOR), which is the maximum bending stress at rupture calculated using simple elastic beam theory for an isotropic, homogeneous material. Because the MOR calculations ignore the composite inhomogeneity, results are somewhat qualitative, but nonetheless useful for establishing environmental and temperature multiplication factors. Six beam bending tests were performed on the 1.5-mm-thick chopped-carbon-fiber composite specimens. Results are summarized in Table 52.

Effects of temperature on uniaxial flexure properties of 3-mm-thick chopped-carbon-fiber composite were investigated in six tests each at $-40^{\circ}, 70^{\circ}$, and $120^{\circ} \mathrm{C}$. Results are presented in Table 53. Temperature multiplication factors (defined as the ratio of at-temperature MOR to the room-temperature MOR value) present the MOR at each temperature in terms of the roomtemperature baseline value.

The effects of fluid environment on uniaxial flexure properties of 3-mm-thick choppedcarbon-fiber composite were assessed in tests on twelve beam specimens: six pre-exposed in $23^{\circ} \mathrm{C}$ distilled water for $1000 \mathrm{~h}$, and six pre-exposed in windshield washer fluid for $100 \mathrm{~h}$. Test results and strength multiplication factors are presented in Tables 54 and 55 for exposures in distilled water and windshield washer fluid, respectively.

\subsubsection{Biaxial flexural properties}

The test specimen together with the support and loading arrangement used for biaxial flexural tests were as described in Chap. 8 of Ref. 4. The specimen outside diameter was $94 \mathrm{~mm}$. The load-ring diameter was $38.1 \mathrm{~mm}$, while the support-ring diameter was $88.9 \mathrm{~mm}$. For the $3-\mathrm{mm}-$ thick composite, six specimens were tested at room temperature, and six at $120^{\circ} \mathrm{C}$. For the 1.5 mm-thick composite, three tests were conducted at $23^{\circ} \mathrm{C}$ and three tests, at $120^{\circ} \mathrm{C}$. Results are summarized in Tables 56 and 57 for the 3-mm-thick and the 1.5-mm-thick composites, 
respectively. Biaxial flexure results are presented in terms of the failure load and used in this way to establish temperature multiplication factors, which are defined as the ratio of the attemperature failure load to the average room-temperature failure load.

To explore fluid effects for the 3-mm-thick composite, six specimens presoaked for $100 \mathrm{~h}$ in windshield washer fluid and six specimens presoaked for $1000 \mathrm{~h}$ in room-temperature distilled water were also tested. All tests were conducted in the laboratory air environment. Results are summarized in Table 58.

\section{SUMMARY}

\subsection{CHOPPED-GLASS-FIBER COMPOSITE}

- The present material exhibits no consistent strong or weak direction.

- Basic elastic constants and strength properties were established over the temperature range of interest.

- Effects of exposure in room-temperature distilled water and windshield washer fluid were investigated.

- Uniaxial flexure properties were established

- Effects of temperature on flexure were explored in tests conducted at $23^{\circ}, 50^{\circ}$, and $120^{\circ} \mathrm{C}$. Effects of fluid environment on flexure properties were assessed for standard exposure conditions.

\subsection{CHOPPED-CARBON-FIBER COMPOSITE}

- Tensile stiffness and strength increase with specimen width. A width factor was provided to permit UTS adjustment for specimens of various widths.

- Room-temperature stiffness values in tension and compression are comparable. Roomtemperature tensile strength is approximately 1.2 times compressive strength.

- Compressive strength values obtained from standard small compressive specimens were nearly identical to those obtained in tests of compression-after-impact specimens. Thus, the short-gage-length compressive specimen gives representative results.

- The room-temperature properties of the chopped-carbon-fiber composite exhibit a high degree of scatter. Coefficients of variation associated with the basic properties range from 15 to $23 \%$. Shear strength, with a coefficient of variation of $8.85 \%$ represents an exception. Results demonstrate that basic properties can vary by as much as a factor of 2.7. Large variations in properties were observed not only between different plaques but also within each individual plaque.

- Basic properties at different temperatures were established.

- Strain rate effects were found to be relatively small, except at the fastest rate examined.

- Prior thermal cycling was found to have a significant effect on shear properties.

- Prior cooling to $-40^{\circ} \mathrm{C}$ did not degrade strength permanently. Once the material is returned to room temperature, the room-temperature strength is recovered.

- Effects of fluid environments on basic mechanical properties were evaluated.

- Uniaxial and biaxial flexure tests were conducted. Effects of temperature and environment on uniaxial and biaxial flexure were explored. 


\section{REFERENCES}

1. J. M. Corum, R L. Battiste, W. Ren, and M. B. Ruggles, Recommended Minimum Test Requirements and Test Methods for Assessing Durability of Random-Glass-Fiber Composites, ORNL-6953, Oak Ridge National Laboratory, Oak Ridge, Tenn., 1999.

2. J. M. Corum, R. L. Battiste, W. Ren, and M. B. Ruggles, Durability-Based Design Criteria for a Chopped-Glass-Fiber Automotive Structural Composite, ORNL/TM-1999/182, Oak Ridge National Laboratory, Oak Ridge, Tenn., 1999.

3. ASTM Standard D3410-87, Standard Test Method for Compressive Properties of Unidirectional or Crossply Fiber-Resin Composites, American Society for Testing and Materials.

4. J. M. Corum, R. L. Battiste, A. Ionita, M. B. Ruggles-Wrenn, and Y. J. Weitsman, Durability-Based Design Criteria for a Chopped-Carbon-Fiber Automotive Composite, ORNL/TM-2003/86, Oak Ridge National Laboratory, Oak Ridge, Tenn., 2003.

5. ASTM Standard D 790, Test Methods for Flexural Properties of Unreinforced and Reinforced Plastics and Electrical Insulating Materials, American Society for Testing and Materials. 
Table 1. Summary of room-temperature stiffness values for the chopped glass-fiber composite

\begin{tabular}{|c|c|c|c|c|c|c|c|}
\hline $\begin{array}{c}\text { Specimen } \\
\text { number }\end{array}$ & $\begin{array}{c}\text { Stiffness } \\
\text { (GPa) }\end{array}$ & $\begin{array}{l}\text { Specimen } \\
\text { number }\end{array}$ & $\begin{array}{c}\text { Stiffness } \\
\text { (GPa) }\end{array}$ & $\begin{array}{l}\text { Specimen } \\
\text { number }\end{array}$ & $\begin{array}{c}\text { Stiffness } \\
\text { (GPa) }\end{array}$ & $\begin{array}{c}\text { Specimen } \\
\text { number }\end{array}$ & $\begin{array}{c}\text { Stiffness } \\
\text { (GPa) }\end{array}$ \\
\hline B1-2 & 11.7 & B1-48 & 11.3 & B2-39 & 13.4 & B3-28 & 12.1 \\
\hline B1-3 & 12.1 & B1-49 & 9.58 & B2-40 & 11.7 & B3-29 & 10.8 \\
\hline B1-4 & 11.2 & B1-50 & 11.3 & B2-41 & 14.0 & B3-30 & 11.1 \\
\hline B1-5 & 11.9 & B1-51 & 11.8 & B2-42 & 12.6 & B3-31 & 10.5 \\
\hline B1-6 & 11.9 & B1-52 & 11.6 & B2-43 & 11.2 & B3-32 & 11.8 \\
\hline B1-7 & 11.7 & B1-53 & 11.6 & B2-44 & 10.9 & B3-33 & 13.8 \\
\hline B1-8 & 11.0 & B1-54 & 11.8 & B2-45 & 13.2 & B3-34 & 10.7 \\
\hline B1-9 & 11.1 & B1-55 & 11.2 & B2-46 & 12.2 & B3-35 & 10.6 \\
\hline B1-10 & 10.7 & B1-56 & 11.7 & B2-47 & 13.0 & B3-36 & 12.0 \\
\hline B1-11 & 10.8 & B1-57 & 11.9 & B2-48 & 12.8 & B3-37 & 11.2 \\
\hline B1-12 & 11.0 & B1-58 & 11.0 & B2-49 & 12.8 & B3-38 & 9.86 \\
\hline B1-13 & 10.6 & B2-3 & 12.6 & B2-50 & 11.5 & B3-39 & 12.1 \\
\hline B1-14 & 10.6 & B2-4 & 14.0 & B2-51 & 12.1 & B3-40 & 11.8 \\
\hline B1-15 & 12.1 & B2-5 & 12.4 & B2-52 & 14.3 & B3-41 & 11.2 \\
\hline B1-16 & 11.8 & B2-6 & 11.4 & B2-53 & 12.7 & B3-42 & 11.6 \\
\hline B1-17 & 13.2 & B2-7 & 14.3 & B2-54 & 10.5 & B3-43 & 11.3 \\
\hline B1-18 & 10.4 & B2-8 & 14.8 & B2-55 & 13.6 & B3-44 & 11.1 \\
\hline B1-19 & 12.6 & B2-9 & 13.2 & B2-56 & 13.0 & B3-45 & 12.2 \\
\hline B1-20 & 12.1 & B2-10 & 11.6 & B2-57 & 11.0 & B3-46 & 12.3 \\
\hline B1-21 & 11.0 & B2-11 & 11.9 & B2-58 & 11.2 & B3-47 & 11.8 \\
\hline B1-22 & 8.96 & B2-12 & 13.0 & B3-2 & 11.0 & B3-48 & 10.9 \\
\hline B1-23 & 11.5 & B2-13 & 11.2 & B3-3 & 11.8 & B3-49 & 10.8 \\
\hline B1-24 & 11.2 & B2-14 & 12.1 & B3-4 & 13.0 & B3-50 & 11.7 \\
\hline B1-25 & 11.9 & B2-15 & 11.4 & B3-5 & 12.4 & B3-51 & 11.0 \\
\hline B1-26 & 11.7 & B2-16 & 11.9 & B3-6 & 12.9 & B3-52 & 13.4 \\
\hline B1-27 & 11.2 & B2-17 & 14.7 & B3-7 & 11.0 & B3-53 & 11.0 \\
\hline B1-28 & 11.3 & B2-18 & 11.8 & B3-8 & 13.6 & B3-54 & 9.93 \\
\hline B1-29 & 9.93 & B2-20 & 11.8 & B3-9 & 12.0 & B3-55 & 12.0 \\
\hline B1-30 & 12.8 & B2-21 & 12.9 & B3-10 & 12.2 & B3-56 & 13.7 \\
\hline B1-31 & 11.7 & B2-22 & 12.6 & B3-11 & 12.0 & B3-57 & 11.2 \\
\hline B1-32 & 9.79 & B2-23 & 11.4 & B3-12 & 12.8 & B3-58 & 13.0 \\
\hline B1-33 & 11.4 & B2-24 & 13.0 & B3-13 & 10.9 & B4-2 & 11.7 \\
\hline B1-34 & 12.2 & B2-25 & 12.9 & B3-14 & 12.1 & B4-3 & 12.3 \\
\hline B1-35 & 12.5 & B2-26 & 12.4 & B3-15 & 12.7 & B4-4 & 13.3 \\
\hline B1-36 & 12.7 & B2-27 & 12.1 & B3-16 & 11.9 & B4-5 & 12.8 \\
\hline B1-37 & 11.4 & B2-28 & 13.0 & B3-17 & 10.8 & B4-6 & 11.0 \\
\hline B1-38 & 11.7 & B2-29 & 14.1 & B3-18 & 12.5 & B4-7 & 12.1 \\
\hline B1-39 & 13.0 & B2-30 & 12.5 & B3-19 & 12.3 & B4-8 & 12.9 \\
\hline B1-40 & 12.3 & B2-31 & 13.7 & B3-20 & 11.6 & B4-9 & 11.5 \\
\hline B1-41 & 12.3 & B2-32 & 13.0 & B3-21 & 12.6 & B4-10 & 12.1 \\
\hline B1-42 & 11.4 & B2-33 & 13.2 & B3-22 & 12.1 & B4-11 & 12.9 \\
\hline B1-43 & 12.1 & B2-34 & 11.3 & B3-23 & 11.4 & B4-12 & 12.0 \\
\hline B1-44 & 10.4 & B2-35 & 13.0 & B3-24 & 11.2 & B4-13 & 10.9 \\
\hline B1-45 & 10.5 & B2-36 & 12.0 & B3-25 & 11.4 & B4-14 & 12.3 \\
\hline B1-46 & 13.0 & B2-37 & 12.5 & B3-26 & 11.6 & B4-15 & 12.8 \\
\hline B1-47 & 11.2 & B2-38 & 13.2 & B3-27 & 12.1 & B4-16 & 11.9 \\
\hline
\end{tabular}


Table 1. Continued. Summary of room-temperature stiffness values for the chopped glassfiber composite

\begin{tabular}{|c|c|c|c|c|c|c|c|}
\hline $\begin{array}{c}\text { Specimen } \\
\text { number }\end{array}$ & $\begin{array}{c}\text { Stiffness } \\
(\mathrm{GPa})\end{array}$ & $\begin{array}{c}\text { Specimen } \\
\text { number }\end{array}$ & $\begin{array}{c}\text { Stiffness } \\
(\text { GPa })\end{array}$ & $\begin{array}{c}\text { Specimen } \\
\text { number }\end{array}$ & $\begin{array}{c}\text { Stiffness } \\
(\mathrm{GPa})\end{array}$ & \begin{tabular}{|c|} 
Specimen \\
number
\end{tabular} & $\begin{array}{c}\text { Stiffness } \\
\text { (GPa) }\end{array}$ \\
\hline $\bar{B} 4-17$ & 11.7 & B5-6 & 10.1 & B5-52 & 12.3 & B6-40 & 11.3 \\
\hline B4-18 & 12.3 & B5-7 & 11.0 & B5-53 & 11.4 & B6-41 & 10.5 \\
\hline B4-19 & 11.6 & B5-8 & 13.9 & B5-54 & 10.3 & B6-42 & 11.1 \\
\hline B4-20 & 9.79 & B5-9 & 11.4 & B5-55 & 10.8 & B6-43 & 11.0 \\
\hline B4-21 & 13.4 & B5-10 & 10.8 & B5-56 & 12.2 & B6-44 & 11.7 \\
\hline B4-22 & 11.8 & B5-11 & 11.2 & B5-57 & 11.3 & B6-45 & 11.0 \\
\hline B4-23 & 11.3 & B5-12 & 12.2 & B5-58 & 9.65 & B6-46 & 11.0 \\
\hline B4-24 & 11.6 & B5-13 & 11.1 & B6-1 & 11.6 & B6-47 & 10.2 \\
\hline B4-25 & 12.6 & B5-14 & 10.7 & B6-2 & 10.3 & B6-48 & 10.8 \\
\hline B4-26 & 12.1 & B5-15 & 11.9 & B6-3 & 10.8 & B6-49 & 10.2 \\
\hline B4-27 & 12.4 & B5-16 & 11.6 & B6-4 & 12.3 & B6-50 & 10.9 \\
\hline B4-28 & 11.9 & B5-17 & 11.0 & B6-5 & 11.5 & B6-51 & 10.3 \\
\hline B4-29 & 12.3 & B5-18 & 12.4 & B6-6 & 10.1 & B6-52 & 11.5 \\
\hline B4-30 & 11.4 & B5-19 & 11.0 & B6-7 & 11.4 & B6-53 & 10.3 \\
\hline B4-31 & 10.8 & B5-20 & 10.8 & B6-8 & 11.0 & B6-54 & 10.6 \\
\hline B4-32 & 11.1 & B5-21 & 11.7 & B6-9 & 10.9 & B6-55 & 11.4 \\
\hline B4-33 & 11.1 & B5-22 & 11.9 & B6-10 & 11.1 & B6-56 & 10.1 \\
\hline B4-34 & 11.9 & B5-23 & 10.6 & B6-11 & 11.0 & B6-57 & 9.93 \\
\hline B4-35 & 9.58 & B5-24 & 10.6 & B6-12 & 12.0 & B6-58 & 9.38 \\
\hline B4-36 & 11.2 & B5-25 & 11.3 & B6-13 & 10.1 & B7-1 & 12.9 \\
\hline B4-37 & 10.4 & B5-26 & 10.4 & B6-14 & 11.9 & B7-2 & 10.8 \\
\hline B4-38 & 10.0 & B5-27 & 10.6 & B6-15 & 10.9 & B7-3 & 12.0 \\
\hline B4-39 & 11.1 & B5-28 & 10.7 & B6-16 & 10.5 & B7-4 & 13.4 \\
\hline B4-40 & 11.8 & B5-29 & 10.5 & B6-17 & 9.79 & B7-5 & 11.8 \\
\hline B4-41 & 10.2 & B5-30 & 13.4 & B6-18 & 12.1 & B7-6 & 12.6 \\
\hline B4-42 & 12.0 & B5-31 & 10.3 & B6-19 & 11.5 & B7-7 & 13.6 \\
\hline B4-43 & 11.9 & B5-32 & 10.5 & B6-20 & 8.83 & B7-8 & 13.0 \\
\hline B4-44 & 11.2 & B5-33 & 11.5 & B6-21 & 11.9 & B7-9 & 10.4 \\
\hline B4-45 & 11.7 & B5-34 & 11.1 & B6-22 & 10.3 & B7-10 & 11.0 \\
\hline B4-46 & 10.8 & B5-35 & 10.3 & B6-23 & 11.1 & B7-11 & 12.5 \\
\hline B4-47 & 9.86 & B5-36 & 10.8 & B6-24 & 11.7 & B7-12 & 11.9 \\
\hline B4-48 & 11.1 & B5-37 & 10.6 & B6-25 & 10.2 & B7-13 & 11.8 \\
\hline B4-49 & 11.5 & B5-38 & 10.6 & B6-26 & 11.2 & B7-14 & 13.5 \\
\hline B4-50 & 10.1 & B5-39 & 11.2 & B6-27 & 11.8 & B7-15 & 11.5 \\
\hline B4-51 & 11.1 & B5-40 & 10.5 & B6-28 & 11.0 & B7-16 & 11.0 \\
\hline B4-52 & 11.3 & B5-41 & 11.9 & B6-29 & 10.7 & B7-17 & 11.6 \\
\hline B4-53 & 11.0 & B5-42 & 11.1 & B6-30 & 12.3 & B7-18 & 12.5 \\
\hline B4-54 & 10.5 & B5-43 & 11.4 & B6-31 & 10.7 & B7-19 & 12.7 \\
\hline B4-55 & 11.1 & B5-44 & 10.6 & B6-32 & 11.0 & B7-20 & 11.8 \\
\hline B4-57 & 11.0 & B5-45 & 11.7 & B6-33 & 10.5 & B7-21 & 12.8 \\
\hline B4-58 & 9.38 & B5-46 & 11.9 & B6-34 & 11.9 & B7-22 & 12.4 \\
\hline B5-1 & 11.0 & B5-47 & 9.86 & B6-35 & 9.86 & B7-23 & 13.0 \\
\hline B5-2 & 9.90 & B5-48 & 11.7 & B6-36 & 11.1 & B7-24 & 13.6 \\
\hline B5-3 & 11.9 & B5-49 & 11.2 & B6-37 & 10.8 & B7-25 & 12.7 \\
\hline B5-4 & 11.5 & B5-50 & 11.4 & B6-38 & 10.1 & B7-26 & 11.2 \\
\hline B5-5 & 11.7 & B5-51 & 10.6 & B6-39 & 10.7 & B7-27 & 12.7 \\
\hline
\end{tabular}


Table 1. Continued. Summary of room-temperature stiffness values for the chopped glassfiber composite

\begin{tabular}{|c|c|c|c|c|c|c|c|}
\hline $\begin{array}{c}\text { Specimen } \\
\text { number }\end{array}$ & $\begin{array}{c}\text { Stiffness } \\
\text { (GPa) }\end{array}$ & $\begin{array}{l}\text { Specimen } \\
\text { number }\end{array}$ & $\begin{array}{c}\text { Stiffness } \\
\text { (GPa) }\end{array}$ & $\begin{array}{l}\text { Specimen } \\
\text { number }\end{array}$ & $\begin{array}{c}\text { Stiffness } \\
\text { (GPa) }\end{array}$ & $\begin{array}{c}\text { Specimen } \\
\text { number }\end{array}$ & $\begin{array}{c}\text { Stiffness } \\
\text { (GPa) }\end{array}$ \\
\hline B7-28 & 12.1 & B8-18 & 10.9 & B9-16 & 11.5 & B9-48-2 & 10.8 \\
\hline B7-29 & 11.7 & B8-19 & 13.0 & B9-17-1 & 11.1 & B9-49 & 11.0 \\
\hline B7-30 & 12.4 & B8-20 & 10.7 & B9-17-2 & 10.1 & B9-50-1 & 10.8 \\
\hline B7-31 & 11.5 & B8-21 & 13.2 & B9-18 & 11.7 & B9-50-2 & 10.8 \\
\hline B7-32 & 11.5 & B8-22 & 12.0 & B9-19-1 & 10.7 & B9-51 & 12.1 \\
\hline B7-33 & 12.4 & B8-23 & 12.1 & B9-19-2 & 10.6 & B9-52-1 & 11.7 \\
\hline B7-34 & 12.8 & B8-24 & 12.3 & B9-20 & 10.6 & B9-52-2 & 11.9 \\
\hline B7-35 & 11.7 & B8-25 & 12.4 & B9-21 & 13.3 & B9-53 & 11.6 \\
\hline B7-36 & 13.0 & B8-26 & 10.8 & B9-22-1 & 9.93 & B9-54-1 & 11.2 \\
\hline B7-37 & 12.5 & B8-27 & 12.1 & B9-22-2 & 10.5 & B9-54-2 & 9.93 \\
\hline B7-38 & 11.5 & B8-28 & 13.3 & B9-23 & 13.4 & B9-55 & 11.6 \\
\hline B7-39 & 11.4 & B8-29 & 11.4 & B9-24-1 & 11.6 & B9-56 & 11.9 \\
\hline B7-40 & 12.2 & B8-30 & 13.6 & B9-24-2 & 10.8 & B9-57 & 12.3 \\
\hline B7-41 & 12.0 & B8-32 & 11.9 & B9-25 & 12.6 & B9-58 & 12.3 \\
\hline B7-42 & 12.3 & B8-34 & 11.9 & B9-26-1 & 12.1 & B10-1 & 13.2 \\
\hline B7-43 & 12.0 & B8-36 & 12.8 & B9-26-2 & 12.3 & B10-2 & 10.8 \\
\hline B7-44 & 12.1 & B8-38 & 11.6 & B9-27 & 12.3 & B10-3 & 11.0 \\
\hline B7-45 & 11.7 & B8-39 & 13.5 & B9-28 & 11.7 & B10-4 & 12.3 \\
\hline B7-46 & 12.8 & B8-41 & 11.9 & B9-29 & 12.7 & B10-5 & 12.4 \\
\hline B7-47 & 12.6 & B8-43 & 11.6 & B9-30 & 12.5 & B10-6 & 11.6 \\
\hline B7-48 & 11.4 & B8-45 & 11.8 & B9-31-1 & 12.2 & B10-7 & 11.5 \\
\hline B7-49 & 10.4 & B8-47 & 11.0 & B9-31-2 & 10.7 & B10-8 & 14.5 \\
\hline B7-50 & 11.4 & B8-49 & 12.3 & B9-32 & 11.8 & B10-9 & 11.9 \\
\hline B7-51 & 11.5 & B8-51 & 10.2 & B9-33-1 & 11.9 & B10-10 & 11.7 \\
\hline B7-52 & 12.6 & B8-56 & 11.0 & B9-33-2 & 12.2 & B10-11 & 11.9 \\
\hline B7-53 & 11.8 & B9-1 & 10.8 & B9-34 & 12.2 & B10-12 & 12.3 \\
\hline B7-54 & 11.9 & B9-2 & 10.3 & B9-35-1 & 10.6 & B10-13 & 10.7 \\
\hline B7-55 & 11.5 & B9-3 & 11.7 & B9-35-2 & 11.4 & B10-14 & 12.1 \\
\hline B7-56 & 11.6 & B9-4 & 10.8 & B9-36 & 11.4 & B10-15 & 12.7 \\
\hline B7-57 & 12.1 & B9-5-1 & 11.5 & B9-37-1 & 10.9 & B10-16 & 11.0 \\
\hline B7-58 & 12.7 & B9-5-2 & 11.8 & B9-37-2 & 10.5 & B10-17 & 11.2 \\
\hline B8-3 & 10.3 & B9-6 & 10.8 & B9-38 & 11.4 & B10-18 & 12.1 \\
\hline B8-4 & 13.5 & B9-7-1 & 12.8 & B9-39 & 12.3 & B10-19 & 11.9 \\
\hline B8-5 & 13.5 & B9-7-2 & 13.3 & B9-40-1 & 10.4 & B10-20 & 10.6 \\
\hline B8-6 & 12.3 & B9-8 & 11.9 & B9-40-2 & 10.7 & B10-21 & 14.2 \\
\hline B8-7 & 13.0 & B9-9-1 & 10.5 & B9-41 & 13.0 & B10-22 & 11.0 \\
\hline B8-8 & 12.1 & B9-9-2 & 10.5 & B9-42-1 & 11.0 & B10-23 & 12.5 \\
\hline B8-9 & 10.3 & B9-10 & 11.0 & B9-42-2 & 10.7 & B10-24 & 13.3 \\
\hline B8-10 & 12.4 & B9-11-1 & 12.0 & B9-43 & 11.7 & B10-25 & 12.8 \\
\hline B8-11 & 11.9 & B9-11-2 & 11.2 & B9-44-1 & 11.4 & B10-26 & 11.0 \\
\hline B8-12 & 12.4 & B9-12 & 10.9 & B9-44-2 & 11.2 & B10-27 & 12.0 \\
\hline B8-13 & 11.0 & B9-13-1 & 9.93 & B9-45 & 12.2 & B10-28 & 12.8 \\
\hline B8-14 & 12.2 & B9-13-2 & 9.93 & B9-46-1 & 10.6 & B10-29 & 13.2 \\
\hline B8-15 & 12.5 & B9-14 & 12.6 & B9-46-2 & 11.7 & B10-30 & 12.1 \\
\hline B8-16 & 12.0 & B9-15-1 & 11.4 & B9-47 & 11.2 & B10-31 & 13.2 \\
\hline B8-17 & 11.0 & B9-15-2 & 11.2 & B9-48-1 & 11.4 & B10-32 & 12.8 \\
\hline
\end{tabular}


Table 1. Continued. Summary of room-temperature stiffness values for the chopped glassfiber composite

\begin{tabular}{|c|c|c|c|c|c|c|c|}
\hline $\begin{array}{c}\text { Specimen } \\
\text { number }\end{array}$ & $\begin{array}{c}\text { Stiffness } \\
\text { (GPa) }\end{array}$ & $\begin{array}{l}\text { Specimen } \\
\text { number }\end{array}$ & $\begin{array}{c}\text { Stiffness } \\
\text { (GPa) }\end{array}$ & $\begin{array}{l}\text { Specimen } \\
\text { number }\end{array}$ & $\begin{array}{c}\text { Stiffness } \\
\text { (GPa) }\end{array}$ & $\begin{array}{c}\text { Specimen } \\
\text { number }\end{array}$ & $\begin{array}{c}\text { Stiffness } \\
\text { (GPa) }\end{array}$ \\
\hline B10-33 & 12.1 & B11-22 & 11.9 & B12-10 & 10.2 & B12-56 & 11.8 \\
\hline B10-34 & 12.5 & B11-23 & 11.4 & B12-11 & 11.2 & B12-57 & 12.6 \\
\hline B10-35 & 11.0 & B11-24 & 12.8 & B12-12 & 12.2 & B12-58 & 9.58 \\
\hline B10-36 & 12.1 & B11-25 & 11.0 & B12-13 & 11.4 & B13-2 & 11.2 \\
\hline B10-37 & 12.3 & B11-26 & 11.7 & B12-14 & 11.7 & B13-4 & 13.9 \\
\hline B10-38 & 11.5 & B11-27 & 12.7 & B12-15 & 13.2 & B13-5 & 14.0 \\
\hline B10-39 & 13.4 & B11-28 & 11.4 & B12-16 & 10.9 & B13-7 & 15.2 \\
\hline B10-40 & 10.8 & B11-29 & 12.3 & B12-17 & 11.2 & B13-8 & 13.7 \\
\hline B10-41 & 11.8 & B11-30 & 11.8 & B12-18 & 10.7 & B13-9 & 12.0 \\
\hline B10-42 & 13.4 & B11-31 & 11.8 & B12-19 & 11.3 & B13-10 & 12.1 \\
\hline B10-43 & 12.5 & B11-32 & 11.4 & B12-20 & 12.6 & B13-11 & 12.6 \\
\hline B10-44 & 11.8 & B11-33 & 11.0 & B12-21 & 11.5 & B13-12 & 11.8 \\
\hline B10-45 & 11.4 & B11-34 & 11.1 & B12-22 & 11.7 & B13-13 & 16.2 \\
\hline B10-46 & 11.4 & B11-35 & 11.7 & B12-23 & 12.7 & B13-14 & 13.3 \\
\hline B10-47 & 10.5 & B11-36 & 12.1 & B12-24 & 11.7 & B13-15 & 11.5 \\
\hline B10-48 & 13.5 & B11-37 & 11.9 & B12-25 & 11.5 & B13-16 & 12.2 \\
\hline B10-49 & 12.1 & B11-38 & 11.5 & B12-26 & 11.7 & B13-17 & 14.6 \\
\hline B10-50 & 10.9 & B11-39 & 11.9 & B12-27 & 12.9 & B13-19 & 12.5 \\
\hline B10-51 & 11.0 & B11-40 & 10.5 & B12-28 & 13.0 & B13-20 & 13.3 \\
\hline B10-52 & 12.8 & B11-41 & 10.5 & B12-29 & 12.8 & B13-21 & 11.7 \\
\hline B10-53 & 13.0 & B11-42 & 11.7 & B12-30 & 12.8 & B13-23 & 13.6 \\
\hline B10-54 & 10.1 & B11-43 & 11.3 & B12-31 & 11.0 & B13-24 & 13.6 \\
\hline B10-55 & 12.1 & B11-44 & 11.6 & B12-32 & 10.0 & B13-26 & 12.5 \\
\hline B10-56 & 12.5 & B11-45 & 10.7 & B12-33 & 11.4 & B13-27 & 11.6 \\
\hline B10-57 & 10.8 & B11-46 & 10.3 & B12-34 & 12.0 & B13-28 & 13.0 \\
\hline B10-58 & 12.3 & B11-47 & 11.4 & B12-35 & 11.6 & B13-30 & 13.6 \\
\hline B11-1 & 12.1 & B11-48 & 11.9 & B12-36 & 13.2 & B13-31 & 12.3 \\
\hline B11-2 & 10.2 & B11-49 & 11.7 & B12-37 & 11.9 & B13-32 & 12.2 \\
\hline B11-3 & 11.9 & B11-50 & 11.0 & B12-38 & 12.2 & B13-33 & 13.0 \\
\hline B11-4 & 14.1 & B11-51 & 11.7 & B12-39 & 12.4 & B13-34 & 12.6 \\
\hline B11-6 & 11.4 & B11-52 & 11.2 & B12-40 & 13.5 & B13-35 & 12.0 \\
\hline B11-7 & 12.1 & B11-53 & 10.1 & B12-41 & 13.1 & B13-36 & 11.9 \\
\hline B11-8 & 12.1 & B11-54 & 11.3 & B12-42 & 12.8 & B13-37 & 12.8 \\
\hline B11-9 & 10.6 & B11-55 & 11.9 & B12-43 & 11.6 & B13-38 & 13.2 \\
\hline B11-10 & 11.8 & B11-56 & 11.0 & B12-44 & 11.2 & B13-39 & 12.7 \\
\hline B11-11 & 11.9 & B11-57 & 10.9 & B12-45 & 11.7 & B13-40 & 11.9 \\
\hline B11-12 & 12.1 & B11-58 & 11.0 & B12-46 & 13.3 & B13-41 & 14.3 \\
\hline B11-13 & 9.10 & B12-1 & 12.4 & B12-47 & 10.9 & B13-42 & 11.4 \\
\hline B11-14 & 10.7 & B12-2 & 12.0 & B12-48 & 11.7 & B13-43 & 12.1 \\
\hline B11-15 & 13.6 & B12-3 & 11.9 & B12-49 & 11.5 & B13-44 & 14.5 \\
\hline B11-16 & 11.9 & B12-4 & 13.7 & B12-50 & 10.9 & B13-45 & 12.5 \\
\hline B11-17 & 10.3 & B12-5 & 11.1 & B12-51 & 11.8 & B13-46 & 12.8 \\
\hline B11-18 & 11.8 & B12-6 & 9.41 & B12-52 & 12.0 & B13-47 & 13.1 \\
\hline B11-19 & 11.5 & B12-7 & 14.0 & B12-53 & 10.5 & B13-48 & 13.1 \\
\hline B11-20 & 11.0 & B12-8 & 11.1 & B12-54 & 10.9 & B13-49 & 12.5 \\
\hline B11-21 & 11.6 & B12-9 & 11.4 & B12-55 & 11.7 & B13-50 & 14.5 \\
\hline
\end{tabular}


Table 1. Continued. Summary of room-temperature stiffness values for the chopped glassfiber composite

\begin{tabular}{|c|c|c|c|c|c|c|c|}
\hline $\begin{array}{c}\text { Specimen } \\
\text { number }\end{array}$ & $\begin{array}{c}\text { Stiffness } \\
\text { (GPa) }\end{array}$ & $\begin{array}{l}\text { Specimen } \\
\text { number }\end{array}$ & $\begin{array}{c}\text { Stiffness } \\
\text { (GPa) }\end{array}$ & $\begin{array}{l}\text { Specimen } \\
\text { number }\end{array}$ & $\begin{array}{c}\text { Stiffness } \\
\text { (GPa) }\end{array}$ & $\begin{array}{c}\text { Specimen } \\
\text { number }\end{array}$ & $\begin{array}{c}\text { Stiffness } \\
\text { (GPa) }\end{array}$ \\
\hline B13-51 & 10.1 & B14-43 & 11.9 & B30-3-4 & 9.74 & B31-41 & 11.8 \\
\hline B13-52 & 11.2 & B14-44 & 9.89 & B30-3-5 & 10.5 & B31-42 & 11.4 \\
\hline B13-53 & 12.1 & B14-45 & 11.5 & B30-1-1 & 11.9 & B31-43 & 12.4 \\
\hline B13-55 & 12.3 & B14-46 & 12.4 & B30-1-2 & 12.5 & B31-44 & 12.1 \\
\hline B13-57 & 13.3 & B14-47 & 10.8 & B30-1-4 & 12.0 & B31-45 & 10.7 \\
\hline B13-58 & 15.1 & B14-48 & 10.9 & B30-1-5 & 11.6 & B31-46 & 11.9 \\
\hline B13-61 & 11.4 & B14-49 & 10.8 & B31-1 & 11.8 & B31-47 & 12.9 \\
\hline B13-63 & 12.8 & B14-50 & 10.7 & B31-2 & 12.1 & B31-48 & 11.9 \\
\hline B13-64 & 11.7 & B14-51 & 10.0 & B31-3 & 11.6 & B31-49 & 11.5 \\
\hline B14-1 & 11.8 & B14-52 & 11.1 & B31-4 & 14.2 & B31-50 & 10.8 \\
\hline B14-2 & 11.9 & B14-53 & 12.1 & B31-5 & 11.6 & B31-51 & 10.3 \\
\hline B14-3 & 12.3 & B14-54 & 10.7 & B31-6 & 12.1 & B31-52 & 11.6 \\
\hline B14-9 & 11.1 & B14-55 & 11.4 & B31-7 & 12.3 & B31-53 & 13.0 \\
\hline B14-10 & 12.0 & B14-56 & 11.5 & B31-8 & 12.5 & B31-54 & 11.3 \\
\hline B14-11 & 10.7 & B14-57 & 10.5 & B31-9 & 11.9 & B31-55 & 12.1 \\
\hline B14-12 & 10.8 & B14-58 & 10.0 & B31-10 & 13.3 & B31-56 & 10.9 \\
\hline B14-13 & 11.3 & B18-R1 & 12.2 & B31-11 & 12.8 & B31-57 & 11.0 \\
\hline B14-14 & 11.6 & B18-R2 & 12.4 & B31-12 & 13.4 & B31-58 & 12.3 \\
\hline B14-15 & 13.3 & B18-R3 & 12.3 & B31-13 & 11.3 & B32-3 & 14.1 \\
\hline B14-16 & 11.4 & B19-R1 & 12.9 & B31-14 & 12.6 & B32-4 & 12.4 \\
\hline B14-17 & 11.4 & B19-R2 & 12.0 & B31-15 & 11.4 & B32-5 & 11.4 \\
\hline B14-18 & 11.6 & B19-R3 & 12.1 & B31-16 & 11.5 & B32-6 & 13.4 \\
\hline B14-19 & 11.7 & B20-R1 & 12.9 & B31-17 & 11.8 & B32-7 & 12.2 \\
\hline B14-20 & 11.7 & B20-R2 & 11.2 & B31-18 & 12.0 & B32-8 & 12.5 \\
\hline B14-21 & 13.0 & B21-R1 & 12.8 & B31-19 & 11.1 & B32-9 & 11.1 \\
\hline B14-22 & 11.3 & B21-R2 & 11.6 & B31-20 & 11.2 & B32-10 & 10.3 \\
\hline B14-23 & 12.9 & B21-R3 & 12.1 & B31-21 & 14.3 & B32-11 & 11.9 \\
\hline B14-24 & 13.2 & B22-3-1 & 9.89 & B31-22 & 13.5 & B32-12 & 11.4 \\
\hline B14-26 & 10.7 & B22-3-2 & 11.4 & B31-23 & 11.4 & B32-13 & 12.7 \\
\hline B14-27 & 13.6 & B22-3-4 & 11.4 & B31-24 & 13.5 & B32-14 & 11.2 \\
\hline B14-28 & 13.3 & B22-3-5 & 11.7 & B31-25 & 12.4 & B32-15 & 10.9 \\
\hline B14-29 & 10.5 & B22-1-1 & 11.0 & B31-26 & 12.9 & B32-16 & 11.7 \\
\hline B14-29A & 12.0 & B22-1-2 & 11.3 & B31-27 & 12.5 & B32-17 & 12.4 \\
\hline B14-30 & 11.6 & B22-1-4 & 11.4 & B31-28 & 11.0 & B32-18 & 11.5 \\
\hline B14-31 & 13.1 & B22-1-5 & 12.3 & B31-29 & 11.4 & B32-19 & 11.4 \\
\hline B14-32 & 11.0 & B22-2-4 & 11.8 & B31-30 & 13.2 & B32-20 & 12.1 \\
\hline B14-33 & 11.8 & B22-2-5 & 11.2 & B31-31 & 12.7 & B32-21 & 10.7 \\
\hline B14-34 & 14.1 & B22-4-1 & 10.1 & B31-32 & 12.5 & B32-22 & 12.0 \\
\hline B14-35 & 11.9 & B22-4-2 & 12.4 & B31-33 & 11.9 & B32-24 & 13.2 \\
\hline B14-36 & 12.4 & B22-4-4 & 10.2 & B31-34 & 12.9 & B32-25 & 11.9 \\
\hline B14-37 & 11.6 & B23-1-2 & 11.2 & B31-35 & 12.0 & B32-26 & 11.2 \\
\hline B14-38 & 11.4 & B23-1-4 & 11.2 & B31-36 & 11.3 & B32-27 & 12.0 \\
\hline B14-39 & 12.3 & B23-1-5 & 11.3 & B31-37 & 11.9 & B32-28 & 12.6 \\
\hline B14-40 & 11.8 & B23-3-2 & 11.7 & B31-38 & 11.5 & B32-29 & 12.6 \\
\hline B14-41 & 10.5 & B30-3-1 & 11.5 & B31-39 & 11.6 & B32-30 & 13.5 \\
\hline B14-42 & 10.5 & B30-3-2 & 12.3 & B31-40 & 11.7 & B32-31 & 12.3 \\
\hline
\end{tabular}


Table 1. Continued. Summary of room-temperature stiffness values for the chopped glassfiber composite

\begin{tabular}{lc}
\hline $\begin{array}{c}\text { Specimen } \\
\text { number }\end{array}$ & $\begin{array}{c}\text { Stiffness } \\
\text { (GPa) }\end{array}$ \\
\hline B32-32 & 12.5 \\
B32-33 & 12.7 \\
B32-34 & 13.6 \\
B32-35 & 12.3 \\
B32-36 & 11.9 \\
B32-37 & 12.0 \\
B32-38 & 12.4 \\
B32-39 & 11.4 \\
B32-40 & 11.2 \\
B32-41 & 13.0 \\
B32-42 & 10.2 \\
B32-43 & 11.1 \\
B32-44 & 13.3 \\
B32-45 & 10.9 \\
B32-46 & 12.0 \\
B32-47 & 11.8 \\
B32-48 & 12.8 \\
B32-49 & 11.7 \\
B32-50 & 11.5 \\
B32-51 & 13.3 \\
B32-52 & 11.6 \\
B32-53 & 14.3 \\
B32-54 & 13.0 \\
B32-55 & 11.1 \\
B32-56 & 10.9 \\
B32-57 & 11.7 \\
B32-58 & 11.3 \\
Average & 11.8 \\
SD & 1.00 \\
COV (\%) & 8.49 \\
\hline &
\end{tabular}


Table 2. Summary of room-temperature tensile test results for the chopped glass-fiber composite

\begin{tabular}{|c|c|c|c|c|c|}
\hline $\begin{array}{c}\text { Specimen } \\
\text { number }\end{array}$ & $\begin{array}{c}\text { UTS } \\
(\mathrm{MPa})\end{array}$ & $\begin{array}{c}\text { Failure strain } \\
(\%)\end{array}$ & $\begin{array}{c}\text { Prop. limit } \\
\text { stress (MPa) }\end{array}$ & $\begin{array}{l}\text { Prop. limit } \\
\text { strain }(\%)\end{array}$ & $\begin{array}{c}100 \% \times \text { prop. limit } \\
\text { stress / UTS }\end{array}$ \\
\hline B1-3 & 161 & 1.70 & 31.6 & 0.26 & 19.6 \\
\hline B1-4 & 167 & 2.17 & 38.3 & 0.38 & 22.9 \\
\hline B1-28 & 159 & 1.93 & 33.9 & 0.33 & 21.3 \\
\hline B1-29 & 190 & 2.41 & 48.3 & 0.44 & 25.4 \\
\hline B1-55 & 181 & 2.12 & 36.2 & 0.35 & 20.0 \\
\hline B1-56 & 187 & 2.01 & 29.8 & 0.25 & 15.9 \\
\hline B2-3 & 213 & 2.22 & 29.7 & 0.24 & 13.9 \\
\hline B2-29 & 219 & 2.01 & 29.5 & 0.21 & 13.5 \\
\hline B2-56 & 211 & 2.22 & 18.7 & 0.14 & 8.86 \\
\hline B3-3 & 167 & 1.82 & 44.7 & 0.41 & 26.8 \\
\hline B3-28 & 217 & 2.85 & 46.7 & 0.42 & 21.5 \\
\hline B3-29 & 161 & 1.86 & 32.2 & 0.31 & 20.0 \\
\hline B3-56 & 201 & 1.99 & 43.9 & 0.27 & 21.8 \\
\hline B4-3 & 184 & 1.96 & 36.7 & 0.32 & 19.9 \\
\hline B4-29 & 176 & 1.92 & 37.8 & 0.34 & 21.5 \\
\hline B4-39 & 171 & 2.14 & - & - & - \\
\hline B4-43 & 166 & 1.56 & - & - & - \\
\hline B4-55 & 179 & 2.19 & 31.5 & 0.30 & 17.6 \\
\hline B5-3 & 190 & 2.08 & 39.6 & 0.35 & 20.8 \\
\hline B5-29 & 157 & 1.95 & 29.2 & 0.29 & 18.6 \\
\hline B5-56 & 203 & 2.31 & 37.1 & 0.32 & 18.3 \\
\hline B6-3 & 165 & 2.09 & 38.7 & 0.38 & 23.5 \\
\hline B6-29 & 159 & 2.12 & 33.0 & 0.32 & 20.8 \\
\hline B6-56 & 158 & 2.08 & 32.7 & 0.34 & 20.7 \\
\hline B7-3 & 190 & 2.03 & 40.8 & 0.38 & 21.5 \\
\hline B7-21 & 222 & 2.42 & - & - & - \\
\hline B7-29 & 184 & 1.93 & 27.1 & 0.25 & 14.7 \\
\hline B7-32 & 202 & 2.12 & - & - & - \\
\hline B7-43 & 183 & 1.92 & - & - & - \\
\hline B7-56 & 196 & 2.18 & 33.4 & 0.31 & 17.0 \\
\hline B8-3 & 169 & 2.16 & 43.6 & 0.42 & 25.8 \\
\hline B8-29 & 179 & 2.04 & 39.2 & 0.35 & 21.9 \\
\hline B8-56 & 174 & 2.26 & 33.6 & 0.33 & 19.3 \\
\hline B9-3 & 180 & 2.00 & 34.5 & 0.27 & 19.2 \\
\hline B9-29 & 177 & 1.90 & 34.3 & 0.31 & 19.4 \\
\hline B9-56 & 191 & 2.20 & 33.6 & 0.30 & 17.6 \\
\hline B10-3 & 196 & 2.35 & 27.7 & 0.25 & 14.1 \\
\hline B10-29 & 202 & 1.76 & 38.5 & 0.31 & 19.1 \\
\hline B10-56 & 217 & 2.28 & 42.1 & 0.36 & 19.4 \\
\hline B11-3 & 177 & 1.90 & 27.6 & 0.25 & 15.6 \\
\hline B11-29 & 197 & 2.12 & 25.8 & 0.23 & 13.1 \\
\hline B11-56 & 178 & 2.22 & 32.4 & 0.31 & 18.2 \\
\hline B12-3 & 193 & 1.97 & 37.4 & 0.32 & 19.4 \\
\hline B12-29 & 204 & 2.08 & 32.8 & 0.26 & 16.1 \\
\hline B12-56 & 209 & 2.36 & 33.2 & 0.28 & 15.9 \\
\hline B18-R1 & 195 & 2.09 & 36.0 & 0.30 & 18.5 \\
\hline
\end{tabular}


Table 2. Continued. Summary of room-temperature tensile test results for the chopped glass-fiber composite

\begin{tabular}{|c|c|c|c|c|c|}
\hline $\begin{array}{c}\text { Specimen } \\
\text { number }\end{array}$ & UTS (MPa) & $\begin{array}{c}\text { Failure strain } \\
(\%)\end{array}$ & $\begin{array}{c}\text { Prop. limit } \\
\text { stress (MPa) }\end{array}$ & $\begin{array}{l}\text { Prop. limit } \\
\text { strain }(\%)\end{array}$ & $\begin{array}{c}100 \% \times \text { prop. limit } \\
\text { stress / UTS }\end{array}$ \\
\hline B18-R2 & 206 & 2.36 & 40.3 & 0.34 & 19.6 \\
\hline B18-R3 & 202 & 2.26 & 36.0 & 0.29 & 17.8 \\
\hline B19-R1 & 201 & 2.13 & 32.1 & 0.26 & 16.0 \\
\hline B19-R2 & 184 & 2.08 & 34.7 & 0.31 & 18.9 \\
\hline B19-R3 & 178 & 1.97 & 33.4 & 0.28 & 18.8 \\
\hline B20-R1 & 203 & 2.05 & 33.8 & 0.27 & 16.7 \\
\hline B20-R2 & 173 & 2.05 & 34.7 & 0.31 & 20.1 \\
\hline B21-R1 & 196 & 1.99 & 36.1 & 0.29 & 18.4 \\
\hline B21-R2 & 171 & 1.99 & 35.4 & 0.32 & 20.7 \\
\hline B21-R3 & 188 & 2.01 & 36.0 & 0.30 & 19.1 \\
\hline B31-3 & 179 & 2.09 & - & - & - \\
\hline B31-29 & 185 & 2.05 & - & - & - \\
\hline B31-56 & 168 & 2.34 & - & - & - \\
\hline B32-3 & 188 & 1.63 & - & - & - \\
\hline B32-29 & 199 & 2.24 & - & - & - \\
\hline B32-56 & 165 & 2.25 & - & - & - \\
\hline B13-3 & 155 & 1.81 & - & - & - \\
\hline B13-29 & 168 & 1.81 & - & - & - \\
\hline B13-61 & 160 & 2.39 & - & - & - \\
\hline B13-63 & 175 & 2.21 & - & - & - \\
\hline B13-64 & 151 & 1.62 & - & - & - \\
\hline B14-3 & 178 & 2.00 & - & - & - \\
\hline B14-29 & 153 & 2.02 & - & - & - \\
\hline B14-56 & 185 & 2.20 & - & - & - \\
\hline B22-3-1 & 171 & 1.97 & - & - & - \\
\hline B22-3-2 & 149 & 1.65 & - & - & - \\
\hline B22-3-4 & 155 & 1.71 & - & - & - \\
\hline B22-3-5 & 164 & 1.70 & - & - & - \\
\hline B22-1-1 & 168 & 2.11 & - & - & - \\
\hline B22-1-2 & 150 & 1.73 & - & - & - \\
\hline B22-1-4 & 172 & 2.03 & - & - & - \\
\hline B22-1-5 & 171 & 1.78 & - & - & - \\
\hline B23-3-2 & 166 & 1.78 & - & - & - \\
\hline B23-3-4 & 172 & 2.10 & - & - & - \\
\hline B23-3-5 & 153 & 1.77 & - & - & - \\
\hline B22-2-1 & 153 & 1.95 & - & - & - \\
\hline B22-2-4 & 166 & 1.76 & - & - & - \\
\hline B22-2-5 & 162 & 1.74 & - & - & - \\
\hline B22-4-1 & 132 & 1.81 & - & - & - \\
\hline B22-4-2 & 178 & 1.99 & - & - & - \\
\hline B22-4-4 & 152 & 1.92 & - & - & - \\
\hline B23-1-1 & 163 & 1.64 & - & - & - \\
\hline B23-1-2 & 171 & 2.13 & - & - & - \\
\hline B23-1-4 & 175 & 2.22 & - & - & - \\
\hline B23-1-5 & 162 & 1.93 & - & - & - \\
\hline B30-3-1 & 170 & 1.99 & - & - & - \\
\hline
\end{tabular}


Table 2. Continued. Summary of room-temperature tensile test results for the chopped glass-fiber composite

\begin{tabular}{lccccc}
\hline $\begin{array}{c}\text { Specimen } \\
\text { number }\end{array}$ & UTS (MPa) & $\begin{array}{c}\text { Failure strain } \\
(\%)\end{array}$ & $\begin{array}{c}\text { Prop. limit } \\
\text { stress (MPa) }\end{array}$ & $\begin{array}{c}\text { Prop. limit } \\
\text { strain (\%) }\end{array}$ & $\begin{array}{c}\mathbf{1 0 0 \%} \text { x prop. limit } \\
\text { stress / UTS }\end{array}$ \\
\hline B30-3-2 & 175 & 1.88 & - & - & - \\
B30-3-4 & 154 & 2.21 & - & - & - \\
B30-3-5 & 154 & 1.90 & - & - & - \\
B30-1-1 & 173 & 2.01 & - & - & - \\
B30-1-2 & 179 & 1.85 & - & - & - \\
B30-1-4 & 151 & 1.61 & - & - & - \\
B30-1-5 & 171 & 2.00 & - & - & - \\
& & & & & \\
Average & 178 & 2.02 & 35.0 & 0.31 & 3.9 \\
SD & 18.7 & 0.21 & 5.46 & 0.06 & 17.4 \\
COV (\%) & 10.5 & 10.7 & 15.6 & 18.2 & -2 \\
\hline
\end{tabular}

Table 3. Summary of plaque average tensile properties for the chopped glass-fiber composite

\begin{tabular}{lccccc}
\hline $\begin{array}{c}\text { Plaque Number } \\
\text { ORNL / ACC }\end{array}$ & $\begin{array}{c}\text { Number of } \\
\text { stiffness tests }\end{array}$ & $\begin{array}{c}\text { Average } \\
\text { stiffness (GPa) }\end{array}$ & $\begin{array}{c}\text { Number of } \\
\text { tensile tests }\end{array}$ & $\begin{array}{c}\text { Average UTS } \\
\text { (MPa) }\end{array}$ & $\begin{array}{c}\text { Average failure } \\
\text { strain (\%) }\end{array}$ \\
\hline B1/TBP-30 & 57 & 11.5 & 6 & 174 & 2.06 \\
B2/TBP-35 & 55 & 12.6 & 3 & 214 & 2.15 \\
B3/TBP-36 & 57 & 11.8 & 4 & 187 & 2.13 \\
B4/TBP-37 & 56 & 11.5 & 3 & 180 & 2.02 \\
B5/TBP-39 & 58 & 11.2 & 3 & 183 & 2.11 \\
B6/TBP-40 & 58 & 10.9 & 3 & 161 & 2.10 \\
B7/TBP-41 & 58 & 12.1 & 3 & 190 & 2.05 \\
B8/TBP-42 & 28 & 12.0 & 3 & 174 & 2.15 \\
B9/TBP-43 & 81 & 11.4 & 3 & 183 & 2.03 \\
B10/TBP-45 & 58 & 12.1 & 3 & 205 & 2.13 \\
B11/TBP-46 & 57 & 11.5 & 3 & 184 & 2.08 \\
B12/TBP-47 & 57 & 11.8 & 3 & 202 & 2.14 \\
B13/TBP-48 & 52 & 12.8 & 5 & 162 & 1.97 \\
B14/TBP-49 & 53 & 11.6 & 3 & 172 & 2.07 \\
B18/TBP-55 & 3 & 12.3 & 3 & 201 & 2.24 \\
B19/TBP-56 & 3 & 12.3 & 3 & 188 & 2.06 \\
B20/TBP-57 & 2 & 11.8 & 2 & 187 & 2.09 \\
B21/TBP-58 & 3 & 12.2 & 3 & 185 & 2.00 \\
B22/TBP-59 & 13 & 11.2 & 14 & 160 & 2.16 \\
B23/TBP-60 & 4 & 11.4 & 7 & 166 & 2.04 \\
B30/TBP-68 & 8 & 11.5 & 8 & 166 & 1.94 \\
B31/ & 58 & 11.3 & 3 & 178 & 2.16 \\
B32/ & 55 & 12.5 & 3 & 184 & 2.04 \\
Number of tests & 934 & & & & \\
Overall average & & 11.8 & 94 & 177 & 2.02 \\
SD & & 1.00 & & 18.4 & 0.21 \\
COV (\%) & & 8.50 & & & 10.5 \\
\hline
\end{tabular}


Table 4. Tensile properties of the chopped glass-fiber composite at different temperatures

\begin{tabular}{|c|c|c|c|}
\hline $\begin{array}{c}\text { Specimen } \\
\text { number }\end{array}$ & $\begin{array}{c}\text { Stiffness } \\
\text { (GPa) }\end{array}$ & $\begin{array}{c}\text { UTS } \\
\text { (MPa) }\end{array}$ & $\begin{array}{c}\text { Failure } \\
\text { strain }(\%)\end{array}$ \\
\hline \multicolumn{4}{|c|}{$23^{\circ} \mathrm{C}$} \\
\hline B4-39 & 11.1 & 171 & 2.14 \\
\hline B4-43 & 11.9 & 166 & 1.56 \\
\hline B4-3 & 12.3 & 184 & 1.96 \\
\hline B4-29 & 12.3 & 176 & 1.92 \\
\hline B4-55 & 11.1 & 179 & 2.19 \\
\hline B7-3 & 12.0 & 190 & 2.03 \\
\hline B7-21 & 12.8 & 222 & 2.42 \\
\hline B7-29 & 11.7 & 184 & 1.93 \\
\hline B7-32 & 11.5 & 202 & 2.12 \\
\hline B7-43 & 12.0 & 183 & 1.92 \\
\hline B7-56 & 11.6 & 196 & 2.18 \\
\hline Average & 11.8 & 187 & 2.03 \\
\hline SD & 0.51 & 15.7 & 0.22 \\
\hline $\operatorname{COV}(\%)$ & 4.28 & 8.43 & 10.8 \\
\hline \multicolumn{4}{|c|}{$\mathbf{5 0}^{\circ} \mathrm{C}$} \\
\hline B4-40 & 10.1 & 149 & 1.80 \\
\hline B4-44 & 10.0 & 158 & 1.90 \\
\hline B7-9 & 8.89 & 144 & 2.00 \\
\hline B7-17 & 10.5 & 183 & 2.20 \\
\hline B7-33 & 10.9 & 194 & 2.48 \\
\hline B7-44 & 10.5 & 181 & 2.19 \\
\hline B7-49 & 9.93 & 168 & 2.03 \\
\hline B7-58 & 11.9 & 167 & 1.63 \\
\hline Average & 10.4 & 168 & 2.03 \\
\hline SD & 0.87 & 17.4 & 0.26 \\
\hline $\operatorname{COV}(\%)$ & 8.38 & 10.3 & 13.0 \\
\hline \multicolumn{4}{|c|}{$120^{\circ} \mathrm{C}$} \\
\hline B4-41 & 6.73 & 107 & 1.96 \\
\hline B4-41 & 6.73 & 107 & 1.96 \\
\hline B4-45 & 6.94 & 113 & 2.00 \\
\hline B4-47 & 6.44 & 105 & 1.93 \\
\hline B4-49 & 7.43 & 119 & 1.91 \\
\hline B7-10 & 8.55 & 150 & 1.99 \\
\hline B7-24 & 9.51 & 167 & 2.10 \\
\hline B7-28 & 8.76 & 157 & 2.43 \\
\hline B7-37 & 8.27 & 147 & 2.20 \\
\hline B7-48 & 7.86 & 148 & 2.34 \\
\hline B7-54 & 8.14 & 140 & 2.06 \\
\hline Average & 7.86 & 135 & 2.09 \\
\hline SD & 0.98 & 22.3 & 0.18 \\
\hline $\operatorname{COV}(\%)$ & 12.4 & 16.5 & 8.49 \\
\hline
\end{tabular}


Table 4. Continued. Tensile properties of the chopped glass-fiber composite at different temperatures

\begin{tabular}{lccc}
\hline $\begin{array}{c}\text { Specimen } \\
\text { number }\end{array}$ & $\begin{array}{c}\text { Stiffness } \\
\text { (GPa) }\end{array}$ & $\begin{array}{c}\text { UTS } \\
\text { (MPa) }\end{array}$ & $\begin{array}{c}\text { Failure } \\
\text { strain (\%) }\end{array}$ \\
\hline \multicolumn{3}{c}{$\mathbf{- 4 0}^{\circ}$ C } \\
B4-38 & 12.7 & 181 & 1.88 \\
B4-42 & 13.9 & 202 & 2.19 \\
B4-46 & 12.8 & 172 & 2.39 \\
B4-48 & 12.9 & 197 & 2.27 \\
B7-8 & 15.7 & 229 & 2.00 \\
B7-11 & 14.6 & 207 & 1.90 \\
B7-12 & 14.3 & 205 & 2.26 \\
B7-13 & 14.0 & 199 & 2.12 \\
B7-22 & 14.9 & 219 & 2.02 \\
B7-23 & 14.8 & 258 & 2.61 \\
B7-26 & 13.1 & 175 & 1.88 \\
B7-39 & 13.7 & 225 & 2.79 \\
B7-42 & 14.5 & 224 & 2.35 \\
B7-52 & 16.1 & 260 & 2.43 \\
B7-53 & 14.1 & 204 & 1.98 \\
B7-57 & 14.5 & 210 & 1.81 \\
& & & \\
Average & 14.2 & 211 & 2.18 \\
SD & 0.98 & 25.4 & 0.28 \\
COV (\%) & 6.92 & 12.1 & 13.0 \\
\hline
\end{tabular}


Table 5. Effect of exposure in $50^{\circ} \mathrm{C}$ distilled water on tensile strength and stiffness of the chopped glass-fiber composite in the "as-received" condition

\begin{tabular}{|c|c|c|c|c|c|c|}
\hline $\begin{array}{c}\text { Specimen } \\
\text { number }\end{array}$ & $\begin{array}{c}\text { Virgin stiffness } \\
\text { (GPa) }\end{array}$ & $\begin{array}{c}\text { Stiffness } \\
(\mathrm{GPa})\end{array}$ & $\begin{array}{c}\text { UTS } \\
(\mathrm{MPa})\end{array}$ & $\begin{array}{c}\text { Failure } \\
\text { strain }(\%)\end{array}$ & $\begin{array}{c}\text { Change in } \\
\text { stiffness (\%) }\end{array}$ & $\begin{array}{c}\text { Change in } \\
\text { strength (\%) }\end{array}$ \\
\hline \multicolumn{7}{|c|}{ Exposure Time $=892 \mathrm{~h}$} \\
\hline B10-11 & 11.9 & 10.7 & 149 & 1.89 & -9.88 & -27.4 \\
\hline B10-16 & 11.0 & 10.3 & 150 & 1.97 & -5.66 & -27.0 \\
\hline B10-28 & 12.8 & 11.9 & 161 & 1.79 & -7.53 & -21.3 \\
\hline B11-27 & 12.7 & 12.3 & 164 & 1.71 & -3.26 & -10.8 \\
\hline B11-30 & 11.8 & 11.4 & 149 & 1.64 & -3.51 & -19.1 \\
\hline Average & 12.0 & 11.3 & 155 & 1.80 & -5.97 & -21.1 \\
\hline SD & 0.76 & 0.80 & 7.50 & 0.13 & & \\
\hline $\operatorname{COV}(\%)$ & 6.29 & 7.07 & 4.85 & 7.39 & & \\
\hline \multicolumn{7}{|c|}{ Exposure Time $=2160 \mathrm{~h}$} \\
\hline B7-36 & 13.0 & 11.7 & 133 & 1.40 & -10.1 & -29.8 \\
\hline B7-38 & 11.5 & 10.4 & 132 & 1.61 & -9.58 & -30.3 \\
\hline B7-40 & 12.2 & 11.0 & 135 & 1.47 & -10.2 & -28.9 \\
\hline B7-41 & 12.0 & 10.2 & 124 & 1.51 & -14.9 & -34.6 \\
\hline B10-20 & 10.6 & 9.17 & 127 & 1.74 & -13.6 & -37.9 \\
\hline B10-22 & 11.0 & 10.2 & 131 & 1.65 & -7.50 & -36.1 \\
\hline B10-31 & 13.2 & 11.7 & 154 & 1.57 & -11.0 & -24.9 \\
\hline B11-6 & 11.4 & 10.1 & 124 & 1.47 & -11.4 & -32.4 \\
\hline B11-23 & 11.4 & 10.0 & 113 & 1.40 & -12.7 & -38.7 \\
\hline B11-28 & 11.4 & 11.4 & 129 & 1.33 & 0.61 & -29.7 \\
\hline B11-31 & 11.8 & 11.2 & 139 & 1.51 & -5.26 & -24.6 \\
\hline B11-34 & 11.1 & 10.1 & 132 & 1.62 & -8.70 & -28.2 \\
\hline Average & 11.7 & 10.6 & 131 & 1.52 & -9.53 & -31.4 \\
\hline SD & 0.77 & 0.79 & 9.75 & 0.12 & & \\
\hline $\operatorname{COV}(\%)$ & 6.56 & 7.45 & 7.43 & 7.81 & & \\
\hline \multicolumn{7}{|c|}{ Exposure Time $=2787 \mathrm{~h}$} \\
\hline B10-2 & 10.8 & 9.51 & 125 & 1.62 & -11.5 & -38.8 \\
\hline B10-7 & 11.5 & 10.1 & 129 & 1.59 & -12.0 & -37.0 \\
\hline B10-10 & 11.7 & 9.72 & 133 & 1.74 & -16.6 & -35.3 \\
\hline B11-25 & 11.0 & 9.72 & 118 & 1.48 & -11.3 & -35.7 \\
\hline B11-33 & 11.0 & 9.72 & 127 & 1.62 & -11.9 & -30.7 \\
\hline B11-37 & 11.9 & 10.1 & 132 & 1.65 & -15.0 & -28.4 \\
\hline Average & 11.3 & 9.83 & 127 & 1.62 & -13.1 & -34.3 \\
\hline SD & 0.46 & 0.25 & 5.21 & 0.08 & & \\
\hline $\operatorname{COV}(\%)$ & 4.04 & 2.58 & 4.09 & 5.23 & & \\
\hline
\end{tabular}


Table 5. Continued. Effect of exposure in $50^{\circ} \mathrm{C}$ distilled water on tensile strength and stiffness of the chopped glass-fiber composite in the "as-received" condition

\begin{tabular}{|c|c|c|c|c|c|c|}
\hline $\begin{array}{c}\begin{array}{c}\text { Specimen } \\
\text { number }\end{array} \\
\end{array}$ & $\begin{array}{c}\text { Virgin stiffness } \\
(\mathrm{GPa})\end{array}$ & $\begin{array}{c}\text { Stiffness } \\
(\mathrm{GPa})\end{array}$ & $\begin{array}{c}\text { UTS } \\
\text { (MPa) }\end{array}$ & $\begin{array}{c}\text { Failure } \\
\text { strain }(\%)\end{array}$ & $\begin{array}{c}\text { Change in } \\
\text { stiffness (\%) }\end{array}$ & $\begin{array}{c}\text { Change in } \\
\text { strength (\%) }\end{array}$ \\
\hline \multicolumn{7}{|c|}{ Exposure Time $=6168 \mathrm{~h}$} \\
\hline B10-8 & 14.5 & 12.3 & 127 & 1.25 & -15.3 & -38.0 \\
\hline B10-17 & 11.2 & 9.74 & 119 & 1.59 & -13.4 & -42.1 \\
\hline B10-24 & 13.3 & 11.2 & 123 & 1.45 & -15.5 & -39.9 \\
\hline B11-10 & 11.8 & 10.3 & 114 & 1.40 & -12.7 & -37.9 \\
\hline B11-15 & 12.7 & 11.2 & 113 & 1.24 & -11.4 & -38.6 \\
\hline B11-35 & 11.7 & 9.93 & 103 & 1.29 & -14.8 & -43.9 \\
\hline Average & 12.5 & 10.8 & 117 & 1.37 & -13.9 & -40.1 \\
\hline SD & 1.22 & 0.97 & 8.44 & 0.14 & & \\
\hline $\operatorname{COV}(\%)$ & 9.72 & 8.97 & 7.24 & 10.0 & & \\
\hline \multicolumn{7}{|c|}{ Exposure Time $=6985 \mathrm{~h}$} \\
\hline B10-6 & 11.6 & 10.1 & 105 & 1.32 & -13.3 & -48.7 \\
\hline B10-13 & 10.7 & 9.03 & 107 & 1.60 & -15.5 & -47.9 \\
\hline B10-21 & 14.2 & 12.3 & 119 & 1.08 & -13.6 & -41.9 \\
\hline B11-26 & 11.7 & 10.1 & 105 & 1.26 & -13.3 & -42.9 \\
\hline B11-32 & 11.4 & 9.73 & 102 & 1.39 & -14.5 & -44.7 \\
\hline B11-38 & 11.5 & 10.0 & 109 & 1.19 & -13.2 & -40.8 \\
\hline Average & 11.8 & 10.2 & 108 & 1.31 & -13.9 & -44.5 \\
\hline SD & 1.21 & 1.09 & 6.03 & 0.18 & & \\
\hline $\operatorname{COV}(\%)$ & 10.2 & 10.7 & 5.60 & 13.7 & & \\
\hline \multicolumn{7}{|c|}{ Exposure Time $=6168 \mathrm{~h}$ followed by drying in vacuum at $120^{\circ} \mathrm{C}$ for $2016 \mathrm{~h}$} \\
\hline B10-39 & 13.4 & 13.0 & 175 & 1.77 & -3.09 & -14.5 \\
\hline B10-45 & 11.4 & 11.4 & 162 & 1.78 & -0.06 & -21.1 \\
\hline B11-39 & 11.9 & 11.5 & 152 & 1.86 & -3.41 & -17.5 \\
\hline B11-44 & 11.6 & 11.3 & 138 & 1.51 & -2.02 & -24.9 \\
\hline B11-45 & 10.7 & 10.6 & 129 & 1.62 & -0.90 & -29.9 \\
\hline B11-49 & 11.7 & 11.3 & 131 & 1.40 & -3.44 & -28.9 \\
\hline Average & 11.8 & 11.5 & 148 & 1.66 & -2.16 & -22.8 \\
\hline SD & 0.89 & 0.78 & 18.5 & 0.18 & & \\
\hline $\operatorname{COV}(\%)$ & 7.58 & 6.74 & 12.5 & 10.7 & & \\
\hline
\end{tabular}


Table 6. Effect of exposure in $50^{\circ} \mathrm{C}$ distilled water on tensile strength and stiffness of the chopped glass-fiber composite pre-dried for $912 \mathrm{~h}$ in vacuum at $120^{\circ} \mathrm{C}$

\begin{tabular}{|c|c|c|c|c|c|c|}
\hline $\begin{array}{c}\begin{array}{c}\text { Specimen } \\
\text { number }\end{array} \\
\end{array}$ & $\begin{array}{c}\text { Virgin stiffness } \\
(\text { GPa })\end{array}$ & $\begin{array}{c}\text { Stiffness } \\
(\text { GPa })\end{array}$ & $\begin{array}{c}\text { UTS } \\
\text { (MPa) }\end{array}$ & $\begin{array}{c}\text { Failure } \\
\text { strain }(\%)\end{array}$ & $\begin{array}{c}\text { Change in } \\
\text { stiffness (\%) }\end{array}$ & $\begin{array}{c}\text { Change in } \\
\text { strength (\%) }\end{array}$ \\
\hline \multicolumn{7}{|c|}{ Exposure Time $=0 \mathrm{~h}$} \\
\hline B10-51 & 11.0 & 11.7 & 195 & 2.36 & 5.62 & -4.82 \\
\hline B10-52 & 12.8 & 13.1 & 232 & 2.36 & 2.15 & 13.0 \\
\hline B11-47 & 11.4 & 12.0 & 194 & 2.29 & 5.45 & 5.67 \\
\hline B11-50 & 11.0 & 11.7 & 190 & 2.31 & 6.25 & 3.42 \\
\hline B11-53 & 10.1 & 10.8 & 174 & 2.27 & 6.85 & -5.20 \\
\hline B11-55 & 11.9 & 12.4 & 199 & 2.05 & 4.65 & 7.92 \\
\hline Average & 11.4 & 11.9 & 197 & 2.27 & 5.16 & 3.33 \\
\hline SD & 0.93 & 0.79 & 18.8 & 0.12 & & \\
\hline $\operatorname{COV}(\%)$ & 8.14 & 6.60 & 9.52 & 5.07 & & \\
\hline \multicolumn{7}{|c|}{ Exposure Time $=1268 \mathrm{~h}$} \\
\hline B10-32 & 12.8 & 12.1 & 162 & 1.55 & -5.41 & -20.9 \\
\hline B10-44 & 11.8 & 10.6 & 154 & 1.69 & -9.94 & -24.7 \\
\hline B10-48 & 13.5 & 11.6 & 167 & 1.82 & -14.3 & -18.7 \\
\hline B10-50 & 10.9 & 10.4 & 137 & 1.47 & -4.43 & -33.3 \\
\hline B10-57 & 10.8 & 9.72 & 141 & 1.65 & -13.4 & -28.6 \\
\hline B11-54 & 11.3 & 9.79 & 131 & 1.44 & -9.62 & -31.2 \\
\hline Average & 11.8 & 10.7 & 149 & 1.60 & -9.52 & -26.3 \\
\hline SD & 1.10 & 0.95 & 14.4 & 0.14 & & \\
\hline $\operatorname{COV}(\%)$ & 9.25 & 8.89 & 9.67 & 8.99 & & \\
\hline \multicolumn{7}{|c|}{ Exposure Time $=1895 \mathrm{~h}$} \\
\hline B10-53 & 13.0 & 11.5 & 141 & 1.44 & -11.6 & -31.3 \\
\hline B10-54 & 10.1 & 8.96 & 125 & 1.81 & -11.6 & -39.0 \\
\hline B11-40 & 10.5 & 9.10 & 111 & 1.57 & -13.7 & -39.8 \\
\hline B11-51 & 11.7 & 10.4 & 116 & 1.25 & -11.2 & -36.9 \\
\hline B11-57 & 10.9 & 9.58 & 111 & 1.29 & -12.0 & -39.6 \\
\hline B11-58 & 11.0 & 9.65 & 115 & 1.36 & -11.9 & -37.7 \\
\hline Average & 11.2 & 9.87 & 120 & 1.45 & -12.0 & -37.4 \\
\hline SD & 1.03 & 0.95 & 11.6 & 0.21 & & \\
\hline $\operatorname{COV}(\%)$ & 9.21 & 9.65 & 9.69 & 14.4 & & \\
\hline \multicolumn{7}{|c|}{ Exposure Time $=5276 \mathrm{~h}$} \\
\hline B10-38 & 11.5 & 10.3 & 107 & 1.22 & -10.3 & -47.9 \\
\hline B10-40 & 10.8 & 8.32 & 84.4 & 1.22 & -23.2 & -58.8 \\
\hline B10-42 & 13.4 & 11.7 & 130 & 1.48 & -13.1 & -36.4 \\
\hline B10-47 & 10.5 & 8.96 & 103 & 1.40 & -14.5 & -50.0 \\
\hline B11-41 & 10.5 & 8.57 & 95.7 & 1.37 & -18.2 & -48.0 \\
\hline B11-46 & 10.3 & 9.06 & 103 & 1.43 & -11.8 & -44.3 \\
\hline Average & 11.2 & 9.49 & 104 & 1.35 & -15.2 & -47.6 \\
\hline SD & 1.20 & 1.28 & 15.2 & 0.11 & & \\
\hline $\operatorname{COV}(\%)$ & 10.7 & 13.5 & 14.7 & 8.09 & & \\
\hline
\end{tabular}


Table 6. Continued. Effect of exposure in $50^{\circ} \mathrm{C}$ distilled water on tensile strength and stiffness of the chopped glass-fiber composite pre-dried for $912 \mathrm{~h}$ in vacuum at $120^{\circ} \mathrm{C}$

\begin{tabular}{lcccccc}
\hline $\begin{array}{c}\text { Specimen } \\
\text { number }\end{array}$ & $\begin{array}{c}\text { Virgin stiffness } \\
(\mathbf{G P a})\end{array}$ & $\begin{array}{c}\text { Stiffness } \\
(\mathbf{G P a})\end{array}$ & $\begin{array}{c}\text { UTS } \\
(\mathbf{M P a})\end{array}$ & $\begin{array}{c}\text { Failure } \\
\text { strain (\%) }\end{array}$ & $\begin{array}{c}\text { Change in } \\
\text { stiffness (\%) }\end{array}$ & $\begin{array}{c}\text { Change in } \\
\text { strength (\%) }\end{array}$ \\
\hline & & \multicolumn{6}{c}{ Exposure Time $=\mathbf{6 0 9 2} \mathbf{~ h}$} \\
B10-35 & 11.0 & 9.50 & 103 & 1.28 & -13.8 & -49.9 \\
B10-41 & 11.8 & 10.0 & 104 & 1.27 & -15.2 & -49.2 \\
B10-46 & 11.4 & 9.80 & 103 & 1.39 & -14.5 & -49.6 \\
B11-36 & 12.1 & 10.1 & 101 & 1.38 & -16.6 & -45.3 \\
B11-42 & 11.7 & 9.80 & 103 & 1.61 & -15.9 & -43.8 \\
B11-52 & 11.2 & 9.65 & 103 & 1.36 & -14.1 & -44.2 \\
Average & 11.5 & 9.80 & 103 & 1.38 & -15.0 & -47.0 \\
SD & 0.38 & 0.21 & 1.19 & 0.12 & & \\
COV (\%) & 3.27 & 2.11 & 1.15 & 8.89 & & \\
\hline
\end{tabular}

Table 7. Effect of 2160-h exposure in $50^{\circ} \mathrm{C}$ distilled water on tensile strength and stiffness of the chopped glass-fiber composite at $50^{\circ} \mathrm{C}$

\begin{tabular}{lcccccc}
\hline $\begin{array}{c}\text { Specimen } \\
\text { number }\end{array}$ & $\begin{array}{c}\text { Virgin stiffness } \\
(\mathbf{G P a})\end{array}$ & $\begin{array}{c}\text { Stiffness } \\
(\mathbf{G P a})\end{array}$ & $\begin{array}{c}\text { UTS } \\
(\mathbf{M P a})\end{array}$ & $\begin{array}{c}\text { Failure } \\
\text { strain (\%) }\end{array}$ & $\begin{array}{c}\text { Change in } \\
\text { stiffness (\%) }\end{array}$ & $\begin{array}{c}\text { Change in } \\
\text { strength (\%) }\end{array}$ \\
\hline B7-25 & 12.7 & 10.6 & 128 & 1.56 & -16.5 & -32.6 \\
B7-27 & 12.7 & 10.5 & 127 & 1.44 & -17.3 & -33.2 \\
B7-30 & 12.4 & 10.6 & 127 & 1.61 & -14.5 & -33.2 \\
B7-31 & 11.5 & 9.90 & 114 & 1.40 & -13.9 & -40.0 \\
B7-34 & 12.8 & 10.8 & 129 & 1.61 & -15.6 & -32.1 \\
B7-35 & 11.7 & 9.60 & 122 & 1.77 & -17.9 & -35.8 \\
B7-45 & 11.7 & 10.0 & 114 & 1.35 & -14.5 & -40.0 \\
B7-46 & 12.8 & 10.7 & 128 & 1.39 & -16.4 & -32.6 \\
B7-47 & 12.6 & 10.5 & 127 & 1.43 & -16.7 & -33.2 \\
B7-50 & 11.4 & 9.80 & 113 & 1.52 & -14.0 & -40.5 \\
B7-51 & 11.5 & 9.70 & 117 & 1.47 & -15.7 & -38.4 \\
B7-55 & 11.5 & 9.90 & 115 & 1.62 & -13.9 & -39.5 \\
& & & & & & \\
Average & 12.1 & 10.2 & 122 & 1.51 & -15.6 & -35.9 \\
SD & 0.58 & 0.44 & 6.60 & 0.12 & & \\
COV (\%) & 4.80 & 4.28 & 5.42 & 8.14 & & \\
\hline
\end{tabular}


Table 8. Effect of 1008 -h exposure in $36^{\circ} \mathrm{C}$ distilled water on room-temperature tensile strength and stiffness of the chopped glass-fiber composite

\begin{tabular}{lcccccc}
\hline $\begin{array}{c}\text { Specimen } \\
\text { number }\end{array}$ & $\begin{array}{c}\text { Virgin stiffness } \\
(\mathbf{G P a})\end{array}$ & $\begin{array}{c}\text { Stiffness } \\
(\mathbf{G P a})\end{array}$ & $\begin{array}{c}\text { UTS } \\
\mathbf{( M P a})\end{array}$ & $\begin{array}{c}\text { Failure } \\
\text { strain (\%) }\end{array}$ & $\begin{array}{c}\text { Change in } \\
\text { stiffness (\%) }\end{array}$ & $\begin{array}{c}\text { Change in } \\
\text { strength (\%) }\end{array}$ \\
\hline B6-46 & 11.0 & 10.2 & 147 & 2.12 & -7.75 & -8.95 \\
B6-49 & 10.2 & 9.45 & 146 & 1.91 & -7.36 & -9.59 \\
B6-52 & 11.5 & 10.6 & 148 & 1.88 & -7.72 & -7.97 \\
B31-11 & 12.7 & 11.0 & 166 & 2.17 & -13.3 & -7.00 \\
B31-37 & 11.9 & 10.6 & 165 & 1.86 & -11.4 & -7.35 \\
B31-48 & 11.9 & 10.8 & 170 & 1.97 & -9.07 & -4.44 \\
& & & & & & \\
Average & 11.5 & 10.4 & 157 & 1.99 & -9.43 & -7.55 \\
SD & 0.86 & 0.55 & 11.2 & 0.13 & & \\
COV (\%) & 7.45 & 5.30 & 7.13 & 6.57 & & \\
\hline
\end{tabular}

Table 9. Effect of exposure in $23^{\circ} \mathrm{C}$ distilled water on room-temperature tensile strength and stiffness of the chopped glass-fiber composite

\begin{tabular}{lcccccc}
\hline $\begin{array}{c}\text { Specimen } \\
\text { number }\end{array}$ & $\begin{array}{c}\text { Virgin stiffness } \\
\text { (GPa) }\end{array}$ & $\begin{array}{c}\text { Stiffness } \\
\text { (GPa) }\end{array}$ & $\begin{array}{c}\text { UTS } \\
\text { (MPa) }\end{array}$ & $\begin{array}{c}\text { Failure } \\
\text { strain (\%) }\end{array}$ & $\begin{array}{c}\text { Change in } \\
\text { stiffness (\%) }\end{array}$ & $\begin{array}{c}\text { Change in } \\
\text { strength (\%) }\end{array}$ \\
\hline B8-32 & 11.9 & 11.3 & 183 & 2.18 & -4.36 & -2.34 \\
B8-34 & 11.9 & 11.0 & 177 & 2.01 & -8.15 & -5.43 \\
B8-41 & 11.9 & 11.5 & 181 & 2.19 & -3.37 & -3.07 \\
B8-43 & 11.7 & 11.0 & 185 & 2.22 & -5.80 & -0.91 \\
B8-45 & 11.9 & 11.4 & 178 & 2.23 & -3.55 & -4.89 \\
B8-49 & 12.3 & 11.5 & 186 & 2.09 & -6.70 & -0.59 \\
& & & & & & \\
Average & 11.9 & 11.3 & 182 & 2.15 & -5.32 & -2.87 \\
SD & 0.23 & 0.25 & 3.74 & 0.09 & & \\
COV (\%) & 1.92 & 2.22 & 2.06 & 3.99 & & \\
& & & Exposure Time & $\mathbf{1 0 0 0} \mathbf{h}$ & & \\
B31-18 & 12.0 & 10.8 & 166 & 1.95 & -10.3 & -6.98 \\
B31-19 & 11.1 & 9.77 & 160 & 2.23 & -12.0 & -9.92 \\
B31-20 & 11.2 & 9.79 & 160 & 2.12 & -12.3 & -9.93 \\
B31-42 & 11.4 & 10.5 & 166 & 2.19 & -8.07 & -6.80 \\
B31-43 & 12.4 & 10.4 & 158 & 1.89 & -16.6 & -11.1 \\
B31-44 & 12.1 & 10.7 & 163 & 2.27 & -11.5 & -8.70 \\
& & & & & & \\
Average & 11.7 & 10.3 & 162 & 2.11 & -11.8 & -8.91 \\
SD & 0.55 & 0.44 & 3.10 & 0.16 & & \\
COV (\%) & 4.66 & 4.30 & 1.91 & 7.36 & & \\
\hline
\end{tabular}


Table 10. Effect of exposure in windshield washer fluid on tensile strength and stiffness of the chopped glass-fiber composite

\begin{tabular}{|c|c|c|c|c|c|c|}
\hline $\begin{array}{c}\text { Specimen } \\
\text { number }\end{array}$ & $\begin{array}{c}\text { Virgin stiffness } \\
\text { (GPa) }\end{array}$ & $\begin{array}{c}\text { Stiffness } \\
\text { (GPa) }\end{array}$ & $\begin{array}{l}\text { UTS } \\
\text { (MPa) }\end{array}$ & $\begin{array}{c}\text { Failure } \\
\text { strain }(\%)\end{array}$ & $\begin{array}{l}\text { Stiffness } \\
\text { factor }\end{array}$ & $\begin{array}{l}\text { Strength } \\
\text { factor }\end{array}$ \\
\hline \multicolumn{7}{|c|}{ Exposure Time $=100 \mathrm{~h}$} \\
\hline B11-14 & 10.7 & 10.4 & 163 & 2.06 & 0.97 & 0.89 \\
\hline B11-17 & 10.3 & 9.58 & 170 & 2.33 & 0.93 & 0.92 \\
\hline B11-18 & 11.8 & 10.5 & 178 & 2.32 & 0.89 & 0.97 \\
\hline B11-19 & 11.5 & 10.4 & 174 & 2.21 & 0.90 & 0.95 \\
\hline B11-20 & 11.0 & 10.5 & 171 & 2.23 & 0.96 & 0.93 \\
\hline B11-22 & 11.9 & 11.0 & 173 & 2.20 & 0.92 & 0.94 \\
\hline Average & 11.2 & 10.4 & 172 & 2.23 & 0.93 & 0.93 \\
\hline SD & 0.64 & 0.45 & 4.89 & 0.10 & & \\
\hline $\operatorname{COV}(\%)$ & 5.69 & 4.35 & 2.85 & 4.43 & & \\
\hline \multicolumn{7}{|c|}{ Exposure Time $=1000 \mathrm{~h}$} \\
\hline B11-7 & 12.1 & 10.5 & 172 & 2.51 & 0.87 & 0.93 \\
\hline B11-8 & 12.1 & 11.2 & 169 & 2.33 & 0.93 & 0.92 \\
\hline B11-9 & 10.6 & 9.17 & 159 & 2.49 & 0.87 & 0.86 \\
\hline B11-11 & 11.9 & 10.2 & 175 & 2.50 & 0.86 & 0.95 \\
\hline B11-12 & 12.1 & 10.5 & 162 & 1.85 & 0.87 & 0.88 \\
\hline B11-13 & 9.10 & 7.93 & 143 & 2.41 & 0.87 & 0.78 \\
\hline Average & 11.3 & 9.93 & 163 & 2.35 & 0.88 & 0.89 \\
\hline SD & 1.23 & 1.18 & 11.6 & 0.25 & & \\
\hline $\operatorname{COV}(\%)$ & 10.9 & 11.9 & 7.13 & 10.80 & & \\
\hline \multicolumn{7}{|c|}{ Exposure Time $=2160 \mathrm{~h}$} \\
\hline B7-14 & 13.5 & 12.1 & 160 & 1.91 & 0.90 & 0.84 \\
\hline B7-15 & 11.5 & 9.84 & 170 & 2.20 & 0.86 & 0.89 \\
\hline B7-16 & 11.0 & 9.82 & 163 & 2.19 & 0.89 & 0.86 \\
\hline B7-18 & 12.5 & 10.7 & 175 & 2.60 & 0.86 & 0.92 \\
\hline B7-19 & 12.7 & 10.3 & 164 & 2.32 & 0.81 & 0.86 \\
\hline B7-20 & 11.8 & 10.7 & 168 & 2.30 & 0.91 & 0.88 \\
\hline Average & 12.2 & 10.6 & 167 & 2.25 & 0.87 & 0.88 \\
\hline SD & 0.90 & 0.83 & 5.42 & 0.22 & & \\
\hline $\operatorname{COV}(\%)$ & 7.45 & 7.96 & 3.26 & 9.95 & & \\
\hline
\end{tabular}


Table 11. Summary of in-air room-temperature compression tests for the chopped glassfiber composite

\begin{tabular}{lccc}
\hline $\begin{array}{c}\text { Specimen } \\
\text { number }\end{array}$ & $\mathbf{E}_{\mathbf{C}}(\mathbf{G P a})$ & $\begin{array}{c}\mathbf{U C S} \\
\mathbf{( M P a})\end{array}$ & $\begin{array}{c}\text { Failure } \\
\text { strain (\%) }\end{array}$ \\
\hline $\mathrm{B} 16-21$ & 12.6 & 223 & 2.04 \\
$\mathrm{~B} 16-26$ & 12.5 & 171 & 1.43 \\
$\mathrm{~B} 16-30$ & 12.2 & 210 & 2.26 \\
$\mathrm{~B} 16-31$ & 13.0 & 219 & 1.88 \\
$\mathrm{~B} 16-34$ & 11.8 & 225 & 2.00 \\
$\mathrm{~B} 16-42$ & 12.3 & 210 & 1.78 \\
$\mathrm{~B} 16-47$ & 13.4 & 206 & 1.70 \\
$\mathrm{~B} 16-65$ & 11.7 & 212 & 2.54 \\
$\mathrm{~B} 16-73$ & 9.45 & 210 & 3.04 \\
$\mathrm{~B} 17-04$ & 12.1 & 189 & 1.76 \\
$\mathrm{~B} 17-15$ & 9.72 & 177 & 3.14 \\
$\mathrm{~B} 17-26$ & 8.41 & 135 & 1.73 \\
$\mathrm{~B} 17-33$ & 12.8 & 221 & 2.56 \\
B17-42 & 9.38 & 180 & 2.54 \\
$\mathrm{~B} 17-52$ & 11.3 & 220 & 3.20 \\
$\mathrm{~B} 17-63$ & 11.3 & 113 & 0.95 \\
$\mathrm{~B} 17-73$ & 13.3 & 108 & 0.89 \\
& & & \\
Average & 11.6 & 190 & 2.08 \\
SD & 1.49 & 38.2 & 0.69 \\
COV (\%) & 12.9 & 20.1 & 33.1 \\
\hline
\end{tabular}


Table 12. Compressive properties of the chopped glass-fiber composite at different temperatures

\begin{tabular}{|c|c|c|c|}
\hline $\begin{array}{c}\text { Specimen } \\
\text { number }\end{array}$ & $\mathrm{E}_{\mathrm{C}}(\mathrm{GPa})$ & $\begin{array}{c}\text { UCS } \\
\text { (MPa) }\end{array}$ & $\begin{array}{c}\text { Failure } \\
\text { strain }(\%)\end{array}$ \\
\hline \multicolumn{4}{|c|}{$50^{\circ} \mathrm{C}$} \\
\hline B16-11 & 10.0 & 155 & 2.06 \\
\hline B16-14 & 10.5 & 131 & 1.59 \\
\hline B16-24 & 10.9 & 143 & 1.35 \\
\hline B16-28 & 11.4 & 175 & 1.61 \\
\hline B16-38 & 11.7 & 157 & 1.17 \\
\hline B16-60 & 9.50 & 108 & 0.92 \\
\hline B16-67 & 9.50 & 134 & 2.19 \\
\hline Average & 10.5 & 143 & 1.56 \\
\hline SD & 0.86 & 21.8 & 0.46 \\
\hline $\operatorname{COV}(\%)$ & 8.20 & 15.2 & 29.4 \\
\hline \multicolumn{4}{|c|}{$120^{\circ} \mathrm{C}$} \\
\hline B16-9 & 8.20 & 83.4 & 1.32 \\
\hline B16-10 & 10.4 & 77.7 & 0.58 \\
\hline B16-23 & 8.83 & 89.7 & 1.06 \\
\hline B16-37 & 9.24 & 80.3 & 0.78 \\
\hline B16-43 & 8.07 & 87.5 & 1.41 \\
\hline B16-58 & 8.07 & 84.6 & 1.12 \\
\hline B16-62 & 8.48 & 77.2 & 0.92 \\
\hline Average & 8.76 & 82.9 & 1.03 \\
\hline SD & 0.85 & 4.77 & 0.29 \\
\hline $\operatorname{COV}(\%)$ & 9.68 & 5.76 & 28.7 \\
\hline \multicolumn{4}{|c|}{$-40^{\circ} \mathrm{C}$} \\
\hline B16-12 & 12.6 & 280 & 2.76 \\
\hline B16-18 & 12.3 & 244 & 2.18 \\
\hline B16-25 & 13.2 & 259 & 2.00 \\
\hline B16-33 & 14.3 & 267 & 1.60 \\
\hline B16-36 & 12.3 & 253 & 3.12 \\
\hline B16-41 & 14.4 & 261 & 1.74 \\
\hline B16-57 & 12.5 & 280 & 3.36 \\
\hline B16-69 & 13.4 & 276 & 2.34 \\
\hline Average & 13.1 & 265 & 2.39 \\
\hline SD & 0.85 & 13.1 & 0.64 \\
\hline $\operatorname{COV}(\%)$ & 6.44 & 4.94 & 26.7 \\
\hline
\end{tabular}


Table 13. Effects of exposure in $50^{\circ} \mathrm{C}$ distilled water on room-temperature compressive properties of the chopped glass-fiber composite

\begin{tabular}{|c|c|c|c|c|c|}
\hline $\begin{array}{c}\text { Specimen } \\
\text { number }\end{array}$ & $\begin{array}{c}\text { Compressive } \\
\text { stiffness (GPa) }\end{array}$ & UCS (MPa) & $\begin{array}{c}\text { Failure strain } \\
(\%)\end{array}$ & $\begin{array}{c}\text { Stiffness } \\
\text { factor }\end{array}$ & $\begin{array}{c}\begin{array}{c}\text { Strength } \\
\text { factor }\end{array} \\
\end{array}$ \\
\hline \multicolumn{6}{|c|}{ Exposure time $=912 \mathrm{~h}$} \\
\hline B17-6 & 10.3 & 155 & 1.88 & 0.94 & 0.92 \\
\hline B17-14 & 11.0 & 145 & 1.81 & 1.00 & 0.86 \\
\hline B17-20 & 11.1 & 153 & 1.09 & 1.01 & 0.91 \\
\hline B17-21 & 10.8 & 127 & 0.99 & 0.98 & 0.76 \\
\hline B17-27 & 10.6 & 152 & 1.70 & 0.96 & 0.90 \\
\hline B17-32 & 11.0 & 139 & 1.40 & 1.00 & 0.83 \\
\hline B17-38 & 11.1 & 152 & 1.78 & 1.01 & 0.90 \\
\hline B17-39 & 11.0 & 141 & 0.97 & 1.00 & 0.84 \\
\hline B17-46 & 11.0 & 145 & 1.02 & 1.00 & 0.86 \\
\hline B17-65 & 11.0 & 128 & 1.70 & 1.00 & 0.76 \\
\hline B17-70 & 9.93 & 129 & 1.34 & 0.90 & 0.77 \\
\hline B17-74 & 10.5 & 123 & 1.21 & 0.95 & 0.73 \\
\hline Average & 10.8 & 141 & 1.41 & 0.98 & 0.84 \\
\hline SD & 0.38 & 11.5 & 0.35 & & \\
\hline $\operatorname{COV}(\%)$ & 3.48 & 8.16 & 25.0 & & \\
\hline \multicolumn{6}{|c|}{ Exposure time $=2160 \mathrm{~h}$} \\
\hline B16-32 & 11.9 & 108 & 0.77 & 0.99 & 0.52 \\
\hline B16-35 & 10.2 & 111 & 1.16 & 0.85 & 0.53 \\
\hline B16-40 & 12.0 & 129 & 1.18 & 0.99 & 0.62 \\
\hline B16-48 & 10.3 & 121 & 1.20 & 0.86 & 0.58 \\
\hline B16-49 & 10.7 & 110 & 1.07 & 0.89 & 0.52 \\
\hline B $16-50$ & 11.9 & 113 & 1.02 & 0.99 & 0.54 \\
\hline B16-61 & 12.1 & 114 & 0.89 & 1.00 & 0.54 \\
\hline B16-68 & 10.8 & 110 & 0.96 & 0.89 & 0.52 \\
\hline B17-41 & 9.38 & 126 & 1.51 & 0.89 & 0.60 \\
\hline B $17-43$ & 10.3 & 105 & 1.20 & 0.79 & 0.75 \\
\hline B17-47 & 9.79 & 100 & 1.01 & 0.94 & 0.68 \\
\hline B17-54 & 8.48 & 109 & 1.51 & 0.85 & 0.75 \\
\hline B17-60 & 10.1 & 108 & 1.77 & 0.93 & 0.63 \\
\hline B17-75 & 10.3 & 113 & 0.77 & 0.92 & 0.65 \\
\hline Average & 10.4 & 115 & 1.19 & 0.90 & 0.62 \\
\hline SD & 1.14 & 8.68 & 0.31 & & \\
\hline $\operatorname{COV}(\%)$ & 11.0 & 7.56 & 26.2 & & \\
\hline
\end{tabular}


Table 14. Summary of in-air room-temperature shear tests for the chopped glass-fiber composite

\begin{tabular}{lccc}
\hline Specimen number & G (GPa) & Shear strength (MPa) & Failure strain (\%) \\
\hline B19-S5 & 4.71 & 173 & 4.48 \\
B19-S9 & 4.50 & 164 & 4.97 \\
B19-S10 & 5.22 & 165 & 4.75 \\
B19-S13 & 4.74 & 162 & 4.62 \\
B20-S5 & 5.10 & 165 & 4.71 \\
B20-S8 & 4.94 & 170 & 4.79 \\
B20-S12 & 5.16 & 172 & 4.32 \\
B21-S8 & 4.25 & 166 & 4.46 \\
B21-S9 & 4.67 & 168 & 4.32 \\
B21-S12 & 4.37 & 164 & 5.02 \\
B21-S15 & 5.12 & 161 & 4.78 \\
& & & \\
Average & 4.80 & 166 & 4.66 \\
SD & 0.34 & 3.87 & 0.24 \\
COV (\%) & 6.99 & 2.33 & 5.13 \\
\hline
\end{tabular}

Table 15. Shear properties of the chopped glass-fiber composite at different temperatures Specimen number G(GPa) Shear strength (MPa) Failure strain (\%)

\begin{tabular}{llrl} 
& \multicolumn{3}{c}{$\mathbf{5 0}^{\circ} \mathbf{C}$} \\
B18-S16 & 4.15 & 121 & 3.25 \\
B20-S4 & 4.47 & 135 & 3.71 \\
B20-S6 & 4.35 & 134 & 3.97 \\
B20-S11 & 4.46 & 128 & 4.02 \\
B20-S13 & 4.21 & 131 & 3.43 \\
B21-S3 & 4.03 & 126 & 3.19 \\
Average & 4.28 & 129 & 3.60 \\
SD & 0.18 & 5.18 & 0.36 \\
COV (\%) & 4.19 & 4.01 & 9.99 \\
& & & \\
B18-S1 & 3.26 & 65.6 & 2.41 \\
B18-S12 & 3.24 & 69.9 & 2.94 \\
B20-S2 & 3.65 & 81.8 & 1.98 \\
B20-S10 & 3.77 & 78.3 & 2.06 \\
B20-S16 & 3.54 & 85.8 & 2.19 \\
B21-S1 & 3.00 & 71.6 & 2.31 \\
B21-S6 & 2.87 & 73.4 & 2.44 \\
B21-S16 & 2.83 & 78.7 & 2.47 \\
Average & 3.27 & & \\
SD & 0.36 & 75.6 & 2.35 \\
COV (\%) & 10.9 & 6.68 & 0.30 \\
\hline
\end{tabular}


Table 16. Effects of environment on room-temperature shear properties of the chopped glass-fiber composite

\begin{tabular}{|c|c|c|c|c|c|}
\hline $\begin{array}{c}\text { Specimen } \\
\text { number }\end{array}$ & $\begin{array}{c}\text { Stiffness } \\
(\mathrm{GPa})\end{array}$ & $\begin{array}{c}\text { Shear strength } \\
\text { (MPa) }\end{array}$ & $\begin{array}{c}\text { Failure strain } \\
(\%)\end{array}$ & $\begin{array}{c}\begin{array}{c}\text { Stiffness } \\
\text { factor }\end{array} \\
\end{array}$ & $\begin{array}{r}\text { Strength } \\
\text { factor }\end{array}$ \\
\hline \multicolumn{6}{|c|}{$2160 \mathrm{~h}$ in $50^{\circ} \mathrm{C}$ distilled water } \\
\hline B18-2 & 4.19 & 98.2 & 2.91 & 0.87 & 0.59 \\
\hline B18-3 & 4.20 & 96.9 & 2.72 & 0.88 & 0.58 \\
\hline B18-9 & 4.31 & 103 & 2.97 & 0.90 & 0.62 \\
\hline B18-15 & 4.21 & 98.5 & 2.64 & 0.88 & 0.59 \\
\hline B20-3 & 4.58 & 117 & 1.96 & 0.95 & 0.70 \\
\hline B20-7 & 4.79 & 113 & 2.91 & 1.00 & 0.68 \\
\hline B20-14 & 4.68 & 114 & 2.69 & 0.98 & 0.69 \\
\hline $\mathrm{B} 20-15$ & 4.62 & 111 & 2.78 & 0.96 & 0.67 \\
\hline Average & 4.45 & 107 & 2.70 & 0.93 & 0.64 \\
\hline SD & 0.25 & 8.19 & 0.32 & & \\
\hline $\operatorname{COV}(\%)$ & 5.56 & 7.69 & 11.9 & & \\
\hline \multicolumn{6}{|c|}{$100 \mathrm{~h}$ in windshield washer fluid } \\
\hline B18-4 & & 152 & - & - & 0.92 \\
\hline B18-7 & & 148 & - & - & 0.89 \\
\hline B19-1 & & 170 & - & - & 1.02 \\
\hline B19-4 & & 163 & - & - & 0.98 \\
\hline B19-8 & & 166 & - & - & 1.00 \\
\hline B19-11 & & 164 & - & - & 0.99 \\
\hline Average & & 161 & & & 0.97 \\
\hline SD & & 8.50 & & & \\
\hline $\operatorname{COV}(\%)$ & & 5.29 & & & \\
\hline
\end{tabular}


Table 17. Summary of in-air room-temperature flexure tests for the chopped glass-fiber composite

\begin{tabular}{lccc}
\hline $\begin{array}{c}\text { Specimen } \\
\text { number }\end{array}$ & $\begin{array}{c}\text { Flexural stiffness } \\
\text { (GPa) }\end{array}$ & $\begin{array}{c}\text { MOR } \\
\text { (MPa) }\end{array}$ & $\begin{array}{c}\text { Failure } \\
\text { strain (\%) }\end{array}$ \\
\hline B18-F1 & 12.3 & 337 & 2.50 \\
B18-F8 & 10.5 & 307 & 2.65 \\
B19-F3 & 13.9 & 363 & 2.39 \\
B19-F8 & 13.1 & 319 & 2.22 \\
B20-F1 & 11.2 & 360 & 2.92 \\
B20-F9 & 10.2 & 313 & 2.80 \\
& & & \\
Average & 11.8 & 333 & 2.59 \\
SD & 1.62 & 24.3 & 0.29 \\
COV (\%) & 13.7 & 7.30 & 11.2 \\
\hline
\end{tabular}

Table 18. Flexural properties of the chopped glass-fiber composite at different temperatures

\begin{tabular}{|c|c|c|c|c|c|}
\hline $\begin{array}{c}\text { Specimen } \\
\text { number }\end{array}$ & $\begin{array}{c}\text { Flexural stiffness } \\
\text { (GPa) }\end{array}$ & $\begin{array}{l}\text { MOR } \\
\text { (MPa) }\end{array}$ & $\begin{array}{c}\text { Failure strain } \\
(\%)\end{array}$ & $\begin{array}{c}\begin{array}{c}\text { Stiffness design } \\
\text { factor }\end{array} \\
\end{array}$ & $\begin{array}{c}\text { MOR design } \\
\text { factor }\end{array}$ \\
\hline \multicolumn{6}{|c|}{$5^{\circ}{ }^{\circ} \mathrm{C}$} \\
\hline B19-F2 & 11.2 & 322 & 2.61 & 0.95 & 0.97 \\
\hline B19-F7 & 10.1 & 316 & 2.84 & 0.86 & 0.95 \\
\hline B19-F11 & 10.1 & 315 & 2.83 & 0.86 & 0.95 \\
\hline B20-F2 & 10.8 & 310 & 2.60 & 0.92 & 0.93 \\
\hline B20-F10 & 10.1 & 305 & 2.74 & 0.86 & 0.92 \\
\hline B21-F12 & 11.4 & 320 & 2.54 & 0.97 & 0.96 \\
\hline Average & 10.7 & 315 & 2.69 & 0.90 & 0.94 \\
\hline SD & 0.60 & 6.22 & 0.13 & & \\
\hline $\operatorname{COV}(\%)$ & 5.64 & 1.98 & 4.74 & & \\
\hline \multicolumn{6}{|c|}{$120^{\circ} \mathrm{C}$} \\
\hline B18-F4 & - & 199 & - & - & 0.62 \\
\hline B18-F5 & - & 193 & - & - & 0.60 \\
\hline B19-F4 & - & 205 & - & - & 0.64 \\
\hline B19-F9 & - & 190 & - & - & 0.59 \\
\hline B20-F6 & - & 182 & - & - & 0.54 \\
\hline B20-F8 & - & 171 & - & - & 0.51 \\
\hline Average & & 190 & & & 0.58 \\
\hline SD & & 12.2 & & & \\
\hline $\operatorname{COV}(\%)$ & & 6.39 & & & \\
\hline
\end{tabular}


Table 19. Effects of environment on flexural properties of the chopped glass-fiber composite

\begin{tabular}{|c|c|c|}
\hline $\begin{array}{c}\text { Specimen } \\
\text { number }\end{array}$ & MOR (MPa) & MOR factor \\
\hline \multicolumn{3}{|c|}{2160 in $50^{\circ} \mathrm{C}$ distilled water } \\
\hline B18-F2 & 245 & 0.74 \\
\hline B18-F3 & 237 & 0.71 \\
\hline B18-F9 & 209 & 0.63 \\
\hline B18-F11 & 203 & 0.61 \\
\hline B19-F1 & 216 & 0.65 \\
\hline B19-F6 & 231 & 0.69 \\
\hline B19-F12 & 163 & 0.49 \\
\hline B20-F5 & 200 & 0.60 \\
\hline B20-F7 & 223 & 0.67 \\
\hline B20-F12 & 210 & 0.63 \\
\hline B21-F3 & 221 & 0.66 \\
\hline B21-F4 & 201 & 0.60 \\
\hline B21-F5 & 220 & 0.66 \\
\hline B21-F10 & 239 & 0.72 \\
\hline Average & 216 & 0.65 \\
\hline SD & 20.9 & \\
\hline $\operatorname{COV}(\%)$ & 9.71 & \\
\hline \multicolumn{3}{|c|}{$1000 \mathrm{~h}$ in $23^{\circ} \mathrm{C}$ distilled water } \\
\hline B18-F7 & 316 & 0.95 \\
\hline B21-F1 & 300 & 0.90 \\
\hline B21-F6 & 306 & 0.92 \\
\hline B21-F7 & 307 & 0.92 \\
\hline B21-F11 & 292 & 0.88 \\
\hline B21-F12 & 302 & 0.91 \\
\hline Average & 304 & 0.91 \\
\hline SD & 7.85 & \\
\hline $\operatorname{COV}(\%)$ & 2.58 & \\
\hline \multicolumn{3}{|c|}{$100 \mathrm{~h}$ in windshield washer fluid } \\
\hline B18-F6 & 347 & 1.04 \\
\hline B18-F10 & 308 & 0.93 \\
\hline B18-F12 & 283 & 0.85 \\
\hline B19-F5 & 322 & 0.97 \\
\hline B19-F10 & 345 & 1.03 \\
\hline B20-F4 & 350 & 1.05 \\
\hline B21-F2 & 347 & 1.04 \\
\hline B21-F8 & 327 & 0.98 \\
\hline Average & 329 & 0.98 \\
\hline SD & 23.8 & \\
\hline $\operatorname{COV}(\%)$ & 7.24 & \\
\hline
\end{tabular}


Table 20. Summary of room-temperature stiffness values for the 3-mm-thick chopped carbon-fiber composite

\begin{tabular}{|c|c|c|c|c|c|c|c|}
\hline $\begin{array}{c}\text { Specimen } \\
\text { number }\end{array}$ & $\begin{array}{c}\text { Stiffness } \\
(\mathrm{GPa})\end{array}$ & $\begin{array}{c}\text { Specimen } \\
\text { number }\end{array}$ & $\begin{array}{c}\text { Stiffness } \\
\text { (GPa) }\end{array}$ & $\begin{array}{c}\text { Specimen } \\
\text { number }\end{array}$ & $\begin{array}{c}\text { Stiffness } \\
\text { (GPa) }\end{array}$ & $\begin{array}{c}\begin{array}{c}\text { Specimen } \\
\text { number }\end{array} \\
\end{array}$ & $\begin{array}{c}\text { Stiffness } \\
\text { (GPa) }\end{array}$ \\
\hline$\overline{Z 1-1}$ & 22.1 & Z2-35 & 18.0 & Z7-16 & 24.6 & Z8-4 & 27.6 \\
\hline Z1-2 & 27.7 & Z2-36 & 32.1 & Z7-17 & 32.3 & Z8-5 & 25.6 \\
\hline Z1-3 & 24.5 & Z2-37 & 26.9 & Z7-18 & 36.5 & Z8-6 & 22.2 \\
\hline Z1-4 & 24.1 & Z2-38 & 27.2 & Z7-19 & 26.6 & Z8-7 & 17.3 \\
\hline Z1-5 & 21.5 & Z2-39 & 22.3 & Z7-20 & 26.8 & Z8-8 & 23.2 \\
\hline Z1-6 & 28.3 & Z2-40 & 29.3 & Z7-21 & 35.2 & Z8-9 & 22.5 \\
\hline Z1-7 & 23.0 & Z2-41 & 30.0 & Z7-22 & 32.2 & Z8-10 & 19.5 \\
\hline Z1-8 & 23.7 & Z2-42 & 32.9 & Z7-23 & 32.3 & Z8-11 & 25.1 \\
\hline Z1-9 & 28.5 & Z2-43 & 21.6 & Z7-24 & 29.8 & Z8-12 & 20.8 \\
\hline Z1-10 & 36.1 & Z2-44 & 23.2 & Z7-25 & 31.0 & Z8-13 & 16.7 \\
\hline Z1-11 & 35.8 & Z2-45 & 24.6 & Z7-26 & 36.4 & Z8-14 & 24.1 \\
\hline Z1-12 & 32.0 & Z2-46 & 21.2 & Z7-27 & 30.1 & Z8-15 & 25.9 \\
\hline Z2-1 & 26.1 & Z2-47 & 20.5 & Z7-28 & 28.3 & Z8-16 & 25.6 \\
\hline Z2-2 & 32.0 & Z2-48 & 21.4 & Z7-29 & 29.1 & Z8-17 & 21.9 \\
\hline Z2-3 & 23.2 & Z2-49 & 20.9 & Z7-30 & 28.5 & Z8-18 & 27.0 \\
\hline Z2-4 & 21.0 & Z2-50 & 29.0 & Z7-31 & 34.1 & Z8-19 & 25.7 \\
\hline$Z 2-5$ & 20.4 & Z2-51 & 33.2 & Z7-32 & 32.3 & Z8-20 & 24.5 \\
\hline Z2-6 & 31.0 & Z2-52 & 23.7 & Z7-33 & 32.7 & Z8-21 & 25.4 \\
\hline $\mathrm{Z} 2-7$ & 21.8 & Z2-53 & 20.0 & Z7-34 & 26.4 & Z8-22 & 30.8 \\
\hline Z2-8 & 22.7 & Z2-54 & 25.9 & Z7-35 & 31.2 & Z8-23 & 25.9 \\
\hline Z2-9 & 27.0 & Z2-55 & 31.1 & Z7-36 & 38.5 & Z8-24 & 24.1 \\
\hline $\mathrm{Z} 2-10$ & 20.2 & Z2-56 & 25.2 & Z7-37 & 23.2 & Z8-25 & 22.1 \\
\hline Z2-11 & 27.3 & Z2-57 & 26.2 & Z7-38 & 27.0 & Z8-26 & 21.4 \\
\hline Z2-12 & 22.1 & Z2-58 & 31.4 & Z7-39 & 22.3 & Z8-27 & 26.6 \\
\hline Z2-13 & 27.4 & Z4-1 & 29.6 & Z7-40 & 21.0 & Z8-28 & 27.4 \\
\hline Z2-14 & 24.6 & Z4-2 & 29.5 & Z7-41 & 35.5 & Z8-29 & 28.0 \\
\hline Z2-15 & 29.7 & Z4-3 & 30.5 & Z7-42 & 30.9 & Z8-30 & 26.7 \\
\hline Z2-16 & 28.7 & Z6-1 & 29.3 & Z7-43 & 22.5 & Z8-31 & 23.0 \\
\hline Z2-17 & 27.1 & Z6-2 & 25.1 & Z7-44 & 26.4 & Z8-32 & 26.0 \\
\hline Z2-18 & 24.4 & Z6-3 & 26.8 & Z7-45 & 26.4 & Z8-33 & 23.0 \\
\hline Z2-19 & 25.2 & Z6-4 & 34.2 & Z7-46 & 17.9 & Z8-34 & 23.9 \\
\hline Z2-20 & 27.9 & Z7-1 & 24.1 & Z7-47 & 28.0 & Z8-35 & 30.0 \\
\hline Z2-21 & 24.8 & Z7-2 & 24.1 & Z7-48 & 31.5 & Z8-36 & 29.3 \\
\hline Z2-22 & 26.8 & Z7-3 & 27.6 & Z7-49 & 24.8 & Z8-37 & 23.3 \\
\hline Z2-23 & 29.1 & Z7-4 & 27.7 & Z7-50 & 25.4 & Z8-38 & 28.1 \\
\hline Z2-24 & 31.0 & Z7-5 & 30.1 & Z7-51 & 25.2 & Z8-39 & 33.7 \\
\hline Z2-25 & 27.6 & Z7-6 & 22.9 & Z7-52 & 31.0 & Z8-40 & 27.4 \\
\hline Z2-26 & 27.8 & Z7-7 & 24.3 & Z7-53 & 31.7 & Z8-41 & 23.1 \\
\hline Z2-27 & 32.9 & Z7-8 & 32.4 & Z7-54 & 18.9 & Z8-42 & 23.2 \\
\hline Z2-28 & 33.7 & Z7-9 & 30.1 & Z7-55 & 32.8 & Z8-43 & 23.9 \\
\hline Z2-29 & 31.9 & Z7-10 & 25.9 & Z7-56 & 30.1 & Z8-44 & 26.5 \\
\hline $\mathrm{Z} 2-30$ & 27.4 & Z7-11 & 25.5 & Z7-57 & 33.2 & Z8-45 & 23.7 \\
\hline Z2-31 & 24.1 & Z7-12 & 33.6 & Z7-58 & 18.1 & Z8-46 & 26.4 \\
\hline Z2-32 & 26.5 & Z7-13 & 33.9 & Z8-1 & 28.5 & Z8-47 & 24.2 \\
\hline $\mathrm{Z} 2-33$ & 28.9 & Z7-14 & 27.3 & Z8-2 & 21.2 & Z8-48 & 27.2 \\
\hline Z2-34 & 31.1 & Z7-15 & 39.6 & Z8-3 & 23.0 & Z8-49 & 21.3 \\
\hline
\end{tabular}


Table 20. Continued. Summary of room-temperature stiffness values for the 3-mm-thick chopped carbon-fiber composite

\begin{tabular}{|c|c|c|c|c|c|c|c|}
\hline $\begin{array}{c}\text { Specimen } \\
\text { number }\end{array}$ & $\begin{array}{c}\text { Stiffness } \\
(\mathrm{GPa})\end{array}$ & $\begin{array}{c}\begin{array}{c}\text { Specimen } \\
\text { number }\end{array} \\
\end{array}$ & $\begin{array}{c}\text { Stiffness } \\
(\mathrm{GPa})\end{array}$ & $\begin{array}{c}\begin{array}{c}\text { Specimen } \\
\text { number }\end{array} \\
\end{array}$ & $\begin{array}{c}\text { Stiffness } \\
(\mathrm{GPa})\end{array}$ & $\begin{array}{c}\text { Specimen } \\
\text { number }\end{array}$ & $\begin{array}{c}\text { Stiffness } \\
\text { (GPa) }\end{array}$ \\
\hline$\overline{Z 8-50}$ & 25.9 & Z12-9 & 24.9 & Z12-55 & 27.4 & Z13-47 & 31.6 \\
\hline Z8-51 & 21.9 & Z12-10 & 26.1 & Z12-56 & 27.1 & Z13-48 & 21.9 \\
\hline Z8-52 & 18.9 & Z12-11 & 24.0 & Z12-57 & 20.5 & Z13-49 & 22.4 \\
\hline Z8-53 & 24.7 & Z12-12 & 28.5 & Z13-2 & 26.0 & Z13-50 & 32.4 \\
\hline Z8-54 & 28.3 & Z12-13 & 23.5 & Z13-3 & 30.5 & Z13-51 & 20.9 \\
\hline Z8-55 & 23.8 & Z12-14 & 32.3 & Z13-4 & 26.1 & Z13-52 & 26.1 \\
\hline Z8-56 & 23.9 & Z12-15 & 26.3 & Z13-5 & 21.4 & Z13-53 & 30.0 \\
\hline$Z 8-57$ & 21.2 & Z12-16 & 30.0 & Z13-6 & 27.1 & Z13-54 & 27.5 \\
\hline Z8-58 & 24.4 & Z12-17 & 33.2 & Z13-7 & 30.8 & Z13-55 & 30.3 \\
\hline Z9-1 & 23.6 & Z12-18 & 30.6 & Z13-8 & 30.8 & Z13-56 & 27.8 \\
\hline Z9-2 & 21.0 & Z12-19 & 25.6 & Z13-9 & 29.6 & Z13-57 & 22.1 \\
\hline Z9-3 & 28.9 & Z12-20 & 28.2 & Z13-10 & 26.6 & Z14-2 & 28.2 \\
\hline Z9-4 & 25.5 & Z12-21 & 29.3 & Z13-11 & 24.5 & Z14-3 & 31.2 \\
\hline Z9-5 & 19.0 & Z12-22 & 28.0 & Z13-12 & 28.4 & Z14-4 & 43.6 \\
\hline Z9-6 & 35.1 & Z12-23 & 27.7 & Z13-13 & 23.8 & Z14-5 & 32.3 \\
\hline Z9-7 & 25.6 & Z12-24 & 32.5 & Z13-14 & 32.6 & Z14-6 & 33.1 \\
\hline Z9-8 & 31.5 & Z12-25 & 27.8 & Z13-15 & 26.2 & Z14-7 & 36.7 \\
\hline Z9-9 & 23.9 & Z12-26 & 28.9 & Z13-16 & 21.4 & Z14-8 & 24.8 \\
\hline Z9-10 & 24.5 & Z12-27 & 28.8 & Z13-17 & 26.2 & Z14-9 & 28.4 \\
\hline Z9-11 & 21.0 & Z12-28 & 28.3 & Z13-18 & 33.4 & Z14-10 & 19.9 \\
\hline Z9-12 & 25.9 & Z12-29 & 31.1 & Z13-19 & 25.8 & Z14-11 & 26.2 \\
\hline Z9-13 & 30.4 & Z12-30 & 25.4 & Z13-20 & 23.0 & Z14-12 & 23.4 \\
\hline Z9-14 & 29.0 & Z12-31 & 31.8 & Z13-21 & 33.9 & Z14-13 & 29.5 \\
\hline Z9-15 & 32.1 & Z12-32 & 33.4 & Z13-22 & 31.7 & Z14-14 & 28.6 \\
\hline Z9-16 & 33.6 & Z12-33 & 33.1 & Z13-23 & 30.9 & Z14-15 & 26.2 \\
\hline Z9-17 & 27.4 & Z12-34 & 32.2 & Z13-24 & 27.6 & Z14-16 & 28.1 \\
\hline Z9-18 & 30.4 & Z12-35 & 26.4 & Z13-25 & 23.4 & Z14-17 & 23.2 \\
\hline Z9-19 & 24.3 & Z12-36 & 24.8 & Z13-26 & 31.0 & Z14-18 & 28.8 \\
\hline Z9-20 & 33.6 & Z12-37 & 23.9 & Z13-27 & 25.6 & Z14-19 & 42.1 \\
\hline Z9-21 & 25.1 & Z12-38 & 34.4 & Z13-28 & 30.1 & Z14-20 & 29.7 \\
\hline Z9-22 & 23.0 & Z12-39 & 26.2 & Z13-29 & 31.0 & Z14-21 & 23.0 \\
\hline Z9-23 & 27.6 & Z12-40 & 19.9 & Z13-30 & 33.8 & Z14-22 & 27.0 \\
\hline Z9-24 & 27.2 & Z12-41 & 24.1 & Z13-31 & 26.1 & Z14-23 & 26.8 \\
\hline Z9-25 & 29.7 & Z12-42 & 17.7 & Z13-32 & 30.3 & Z14-24 & 31.3 \\
\hline Z9-26 & 32.8 & Z12-43 & 25.1 & Z13-33 & 29.5 & Z14-25 & 28.8 \\
\hline Z11-1 & 31.2 & Z12-44 & 25.8 & Z13-34 & 23.5 & Z14-26 & 27.2 \\
\hline Z11-2 & 24.0 & Z12-45 & 29.4 & Z13-35 & 32.4 & Z14-27 & 36.8 \\
\hline Z11-3 & 21.7 & Z12-46 & 23.9 & Z13-36 & 30.6 & Z14-28 & 29.9 \\
\hline Z12-1 & 25.5 & Z12-47 & 26.5 & Z13-37 & 27.2 & Z14-29 & 28.1 \\
\hline Z12-2 & 26.2 & Z12-48 & 29.6 & Z13-38 & 23.4 & Z14-30 & 31.1 \\
\hline Z12-3 & 30.3 & Z12-49 & 20.0 & Z13-41 & 27.7 & Z14-31 & 29.0 \\
\hline Z12-4 & 22.9 & Z12-50 & 31.2 & Z13-42 & 23.0 & Z14-32 & 28.3 \\
\hline $\mathrm{Z} 12-5$ & 25.6 & Z12-51 & 23.9 & Z13-43 & 23.1 & Z14-33 & 32.8 \\
\hline Z12-6 & 31.4 & Z12-52 & 27.4 & Z13-44 & 27.0 & Z14-34 & 29.8 \\
\hline Z12-7 & 25.5 & Z12-53 & 28.8 & Z13-45 & 20.6 & Z14-35 & 32.6 \\
\hline Z12-8 & 27.9 & Z12-54 & 36.7 & Z13-46 & 24.5 & Z14-36 & 29.3 \\
\hline
\end{tabular}


Table 20. Continued. Summary of room-temperature stiffness values for the 3-mm-thick chopped carbon-fiber composite

\begin{tabular}{|c|c|c|c|c|c|c|c|}
\hline $\begin{array}{c}\text { Specimen } \\
\text { number }\end{array}$ & $\begin{array}{c}\text { Stiffness } \\
(\mathrm{GPa})\end{array}$ & $\begin{array}{c}\begin{array}{c}\text { Specimen } \\
\text { number }\end{array} \\
\end{array}$ & $\begin{array}{c}\text { Stiffness } \\
(\mathrm{GPa})\end{array}$ & $\begin{array}{c}\text { Specimen } \\
\text { number }\end{array}$ & $\begin{array}{c}\text { Stiffness } \\
(\text { GPa })\end{array}$ & $\begin{array}{c}\text { Specimen } \\
\text { number }\end{array}$ & $\begin{array}{c}\text { Stiffness } \\
\text { (GPa) }\end{array}$ \\
\hline Z14-37 & 35.1 & Z16-26 & 34.7 & Z20-5 & 32.0 & Z20-51 & 37.6 \\
\hline Z14-38 & 31.0 & Z16-27 & 22.3 & Z20-6 & 27.9 & Z20-52 & 30.1 \\
\hline Z14-42 & 25.0 & Z16-28 & 29.1 & Z20-7 & 26.6 & Z20-53 & 27.5 \\
\hline Z14-43 & 21.9 & Z16-29 & 26.7 & Z20-8 & 24.5 & Z20-54 & 34.5 \\
\hline Z14-44 & 16.4 & Z16-30 & 28.5 & Z20-9 & 26.8 & Z20-55 & 26.3 \\
\hline Z14-45 & 22.4 & Z16-31 & 27.9 & Z20-10 & 24.5 & Z20-56 & 26.7 \\
\hline Z14-46 & 24.7 & Z16-32 & 33.6 & Z20-11 & 27.3 & Z20-57 & 34.0 \\
\hline Z14-47 & 25.5 & Z16-33 & 27.4 & Z20-12 & 28.7 & Z20-58 & 32.9 \\
\hline Z14-48 & 31.2 & Z16-34 & 27.8 & Z20-13 & 22.5 & Z23-1 & 29.4 \\
\hline Z14-49 & 34.9 & Z16-35 & 19.1 & Z20-14 & 31.1 & Z23-2 & 29.4 \\
\hline Z14-50 & 28.8 & Z16-36 & 33.0 & Z20-15 & 20.9 & Z23-3 & 24.3 \\
\hline Z14-51 & 32.7 & Z16-37 & 35.0 & Z20-16 & 33.2 & Z24-A1 & 29.9 \\
\hline Z14-52 & 26.1 & Z16-38 & 34.1 & Z20-17 & 31.2 & Z24-A2 & 24.5 \\
\hline Z14-53 & 28.8 & Z16-39 & 23.2 & Z20-18 & 30.4 & Z24-B1 & 26.3 \\
\hline Z14-54 & 30.2 & Z16-40 & 26.3 & Z20-19 & 34.4 & Z24-B2 & 37.9 \\
\hline Z14-55 & 29.9 & Z16-41 & 27.4 & Z20-20 & 34.4 & Z24-C1 & 22.9 \\
\hline Z14-56 & 44.7 & Z16-42 & 28.3 & Z20-21 & 36.2 & Z24-C2 & 28.1 \\
\hline Z14-57 & 25.0 & Z16-43 & 27.0 & Z20-22 & 27.7 & Z24-D1 & 23.4 \\
\hline Z15-1 & 38.0 & Z16-44 & 24.7 & Z20-23 & 31.9 & Z24-D2 & 25.0 \\
\hline Z15-2 & 31.2 & Z16-45 & 27.1 & Z20-24 & 29.4 & Z25-A1 & 33.8 \\
\hline Z15-3 & 26.3 & Z16-46 & 24.2 & Z20-25 & 27.2 & Z25-A2 & 24.3 \\
\hline Z16-1 & 33.1 & Z16-47 & 24.9 & Z20-26 & 28.1 & Z25-B1 & 26.9 \\
\hline Z16-2 & 28.5 & Z16-48 & 26.2 & Z20-27 & 33.2 & Z25-B2 & 26.1 \\
\hline Z16-3 & 20.6 & Z16-49 & 29.0 & Z20-28 & 24.7 & Z25-C1 & 24.9 \\
\hline Z16-4 & 30.0 & Z16-50 & 32.5 & Z20-29 & 33.0 & Z25-C2 & 27.9 \\
\hline Z16-5 & 30.2 & Z16-51 & 25.0 & Z20-30 & 37.6 & Z25-D1 & 27.6 \\
\hline Z16-6 & 25.0 & Z16-52 & 28.0 & Z20-31 & 39.6 & Z25-D2 & 24.6 \\
\hline Z16-7 & 22.3 & Z16-53 & 31.8 & Z20-32 & 29.2 & Z26-1 & 29.0 \\
\hline Z16-8 & 20.9 & Z16-54 & 23.7 & $\mathrm{Z} 20-33$ & 34.2 & Z26-2 & 32.2 \\
\hline Z16-9 & 23.7 & Z16-55 & 25.0 & $\mathrm{Z} 20-34$ & 28.1 & Z26-3 & 29.2 \\
\hline Z16-10 & 27.7 & Z16-56 & 31.2 & Z20-35 & 26.1 & Z26-4 & 26.3 \\
\hline Z16-11 & 26.3 & Z16-57 & 28.1 & Z20-36 & 32.1 & Z26-5 & 23.6 \\
\hline Z16-12 & 31.0 & Z16-58 & 20.9 & $\mathrm{Z} 20-37$ & 32.9 & Z26-6 & 28.6 \\
\hline Z16-13 & 24.7 & Z17-1 & 27.0 & Z20-38 & 23.6 & Z27-1 & 39.6 \\
\hline Z16-14 & 30.0 & Z17-2 & 28.6 & Z20-39 & 27.2 & Z27-2 & 27.4 \\
\hline Z16-15 & 25.2 & Z17-3 & 30.1 & Z20-40 & 26.9 & Z27-3 & 24.0 \\
\hline Z16-16 & 21.4 & Z18-1 & 30.5 & Z20-41 & 24.3 & Z28-1 & 29.2 \\
\hline Z16-17 & 39.6 & Z18-2 & 42.8 & $\mathrm{Z} 20-42$ & 31.0 & Z28-2 & 36.3 \\
\hline Z16-18 & 30.1 & Z18-3 & 23.4 & Z20-43 & 35.0 & Z28-3 & 35.0 \\
\hline Z16-19 & 32.5 & Z19-1 & 32.2 & $\mathrm{Z} 20-44$ & 28.1 & Z28-4 & 28.5 \\
\hline Z16-20 & 27.3 & Z19-2 & 28.2 & Z20-45 & 31.9 & Z28-5 & 20.4 \\
\hline Z16-21 & 31.6 & Z19-3 & 27.9 & Z20-46 & 29.6 & Z28-6 & 26.9 \\
\hline Z16-22 & 31.7 & Z20-1 & 38.3 & $\mathrm{Z} 20-47$ & 31.8 & Z30-1 & 19.4 \\
\hline Z16-23 & 31.4 & Z20-2 & 25.9 & Z20-48 & 27.5 & Z30-2 & 22.2 \\
\hline Z16-24 & 28.5 & Z20-3 & 30.7 & Z20-49 & 23.4 & Z30-3 & 28.0 \\
\hline Z16-25 & 35.4 & Z20-4 & 31.1 & Z20-50 & 32.1 & & \\
\hline
\end{tabular}


Table 20. Continued. Summary of room-temperature stiffness values for the 3-mm-thick chopped carbon-fiber composite

\begin{tabular}{lc}
$\begin{array}{c}\text { Specimen } \\
\text { number }\end{array}$ & $\begin{array}{c}\text { Stiffness } \\
\text { (GPa) }\end{array}$ \\
\hline Average & 27.7 \\
SD & 4.49 \\
COV (\%) & 16.2 \\
MAX & 44.7 \\
MIN & 16.4 \\
\hline
\end{tabular}


Table 21. Summary of room-temperature stiffness values for the 1.5-mm-thick chopped carbon-fiber composite

\begin{tabular}{|c|c|c|c|c|c|c|c|}
\hline $\begin{array}{c}\text { Specimen } \\
\text { number }\end{array}$ & $\mathrm{E}_{1}(\mathrm{GPa})$ & $\mathrm{E}_{2}(\mathrm{GPa})$ & $E_{3}(\mathrm{GPa})$ & $\begin{array}{c}\text { Specimen } \\
\text { number }\end{array}$ & $\mathrm{E}_{1}(\mathrm{GPa})$ & $\mathrm{E}_{2}(\mathrm{GPa})$ & $\overline{E_{3}(\mathrm{GPa})}$ \\
\hline ZZ1-1 & 15.7 & 19.9 & 20.9 & ZZ1-47 & 17.4 & 28.5 & 28.5 \\
\hline ZZ1-2 & 17.7 & 21.5 & 18.5 & ZZ1-48 & 18.4 & 14.9 & 19.2 \\
\hline ZZ1-3 & 28.5 & 19.4 & 30.7 & ZZ1-49 & 19.1 & 22.0 & 15.8 \\
\hline ZZ1-4 & 19.4 & 26.4 & 21.0 & ZZ1-50 & 23.8 & 23.5 & 21.7 \\
\hline ZZ1-5 & 28.5 & 20.9 & 22.4 & ZZ1-51 & 23.6 & 22.5 & 22.3 \\
\hline ZZ1-6 & 21.8 & 19.8 & 17.2 & ZZ1-52 & 18.6 & 27.2 & 21.4 \\
\hline ZZ1-7 & 22.8 & 25.4 & 28.5 & ZZ1-53 & 24.5 & 35.3 & 21.2 \\
\hline ZZ1-8 & 23.1 & 20.5 & 22.3 & ZZ1-54 & 23.0 & 20.3 & 25.6 \\
\hline ZZ1-9 & 27.1 & 28.3 & 22.8 & ZZ1-55 & 32.3 & 32.9 & 25.3 \\
\hline ZZ1-10 & 20.8 & 22.5 & 28.1 & ZZ1-56 & 24.0 & 26.3 & 27.4 \\
\hline ZZ1-11 & 18.5 & 17.4 & 28.3 & ZZ1-57 & 17.9 & 19.5 & 16.8 \\
\hline ZZ1-12 & 16.2 & 13.1 & 22.3 & ZZ1-58 & 18.8 & 15.5 & 19.2 \\
\hline ZZ1-13 & 27.8 & 26.1 & 27.1 & ZZ5-1 & 25.1 & 25.4 & 26.2 \\
\hline ZZ1-14 & 18.3 & 30.5 & 30.9 & ZZ5-2 & 19.0 & 21.4 & 25.4 \\
\hline ZZ1-15 & 25.4 & 26.5 & 31.6 & ZZ5-3 & 27.2 & 26.4 & 26.1 \\
\hline ZZ1-16 & 24.3 & 26.9 & 23.1 & ZZ5-4 & 21.5 & 20.8 & 29.4 \\
\hline ZZ1-17 & 27.0 & 22.3 & 19.3 & ZZ5-5 & 23.9 & 25.9 & 22.7 \\
\hline ZZ1-18 & 21.0 & 20.8 & 33.9 & ZZ5-6 & 20.7 & 20.1 & 23.6 \\
\hline ZZ1-19 & 23.5 & 25.4 & 23.6 & ZZ5-7 & 24.5 & 26.9 & 27.6 \\
\hline ZZ1-20 & 27.7 & 30.9 & 37.4 & ZZ5-8 & 27.3 & 26.5 & 18.7 \\
\hline ZZ1-21 & 21.5 & 13.2 & 24.5 & ZZ5-9 & 28.5 & 22.6 & 25.5 \\
\hline ZZ1-22 & 21.2 & 27.4 & 20.3 & ZZ5-10 & 21.4 & 23.8 & 23.9 \\
\hline ZZ1-23 & 23.0 & 17.2 & 18.3 & ZZ5-11 & 20.2 & 21.4 & 20.7 \\
\hline ZZ1-24 & 24.5 & 25.1 & 25.5 & ZZ5-12 & 21.9 & 26.1 & 31.9 \\
\hline ZZ1-25 & 21.5 & 22.5 & 25.9 & ZZ5-13 & 21.4 & 27.5 & 26.0 \\
\hline ZZ1-26 & 28.2 & 20.3 & 27.2 & ZZ5-14 & 23.9 & 25.9 & 30.1 \\
\hline ZZ1-27 & 30.7 & 18.4 & 14.1 & ZZ5-15 & 25.2 & 18.6 & 24.3 \\
\hline ZZ1-28 & 24.7 & 28.2 & 25.4 & ZZ5-16 & 20.4 & 30.0 & 28.4 \\
\hline ZZ1-29 & 21.8 & 20.2 & 31.9 & ZZ5-17 & 32.6 & 28.2 & 28.0 \\
\hline ZZ1-30 & 28.5 & 31.6 & 30.9 & ZZ5-18 & 23.4 & 30.0 & 29.6 \\
\hline ZZ1-31 & 25.6 & 30.1 & 32.7 & ZZ5-19 & 28.5 & 35.7 & 29.2 \\
\hline ZZ1-32 & 29.6 & 21.9 & 16.7 & ZZ5-20 & 32.1 & 21.3 & 22.6 \\
\hline ZZ1-33 & 36.7 & 22.3 & 25.0 & ZZ5-21 & 28.2 & 19.2 & 27.1 \\
\hline ZZ1-34 & 35.2 & 31.0 & 28.1 & ZZ5-22 & 23.9 & 26.2 & 28.5 \\
\hline ZZ1-35 & 21.3 & 18.6 & 19.5 & ZZ5-23 & 28.1 & 31.8 & 22.8 \\
\hline ZZ1-36 & 21.6 & 20.2 & 21.6 & ZZ5-24 & 23.0 & 25.0 & 31.9 \\
\hline ZZ1-37 & 24.2 & 20.6 & 21.3 & ZZ5-25 & 27.9 & 22.2 & 28.0 \\
\hline ZZ1-38 & 19.7 & 18.8 & 22.3 & ZZ5-26 & 27.6 & 23.6 & 23.4 \\
\hline ZZ1-39 & 24.6 & 14.1 & 15.0 & ZZ5-27 & 24.5 & 30.2 & 34.1 \\
\hline ZZ1-40 & 20.1 & 32.7 & 14.8 & ZZ5-28 & 28.3 & 32.2 & 27.4 \\
\hline ZZ1-41 & 20.6 & 14.2 & 25.2 & ZZ5-29 & 27.1 & 24.8 & 26.5 \\
\hline ZZ1-42 & 24.8 & 22.9 & 26.2 & ZZ5-30 & 27.5 & 27.0 & 35.3 \\
\hline ZZ1-43 & 17.7 & 21.9 & 19.6 & ZZ5-31 & 20.6 & 18.4 & 27.2 \\
\hline ZZ1-44 & 26.8 & 21.6 & 19.1 & ZZ5-32 & 29.3 & 28.4 & 29.0 \\
\hline ZZ1-45 & 31.7 & 26.8 & 19.0 & ZZ5-33 & 22.8 & 23.0 & 21.2 \\
\hline ZZ1-46 & 24.6 & 22.7 & 22.9 & ZZ5-34 & 23.8 & 27.2 & 22.3 \\
\hline
\end{tabular}

Table 21. Continued. Summary of room-temperature stiffness values for 
The 1.5-mm-thick chopped carbon-fiber composite

\begin{tabular}{lccc|lccc}
\hline $\begin{array}{c}\text { Specimen } \\
\text { number }\end{array}$ & $\mathbf{E}_{\mathbf{1}}(\mathbf{G P a})$ & $\mathbf{E}_{\mathbf{2}}(\mathbf{G P a})$ & $\mathbf{E}_{\mathbf{3}}(\mathbf{G P a})$ & $\begin{array}{l}\text { Specimen } \\
\text { number }\end{array}$ & $\mathbf{E}_{\mathbf{1}}(\mathbf{G P a})$ & $\mathbf{E}_{\mathbf{2}}(\mathbf{G P a})$ & $\mathbf{E}_{\mathbf{3}}(\mathbf{G P a})$ \\
\hline ZZ5-35 & 36.1 & 30.6 & 20.8 & ZZ2-3 & - & 19.6 & - \\
ZZ5-36 & 28.1 & 21.8 & 22.6 & ZZ2-4 & - & 20.4 & - \\
ZZ5-37 & 24.1 & 20.8 & 20.1 & ZZ2-5 & - & 22.6 & - \\
ZZ5-38 & 20.4 & 21.1 & 26.2 & ZZ2-6 & - & 26.2 & - \\
ZZ5-40 & 36.3 & 20.8 & 20.3 & ZZ2-7 & - & 35.1 & - \\
ZZ5-41 & 33.7 & 34.5 & 20.6 & ZZ2-8 & - & 24.8 & - \\
ZZ5-42 & 23.7 & 20.5 & 27.7 & ZZ3-1 & - & 22.8 & - \\
ZZ5-43 & 24.0 & 22.4 & 20.1 & ZZ3-2 & - & 22.2 & - \\
ZZ5-44 & 20.9 & 19.0 & 23.8 & ZZ3-3 & - & 26.8 & - \\
ZZ5-45 & 26.3 & 26.5 & 26.3 & ZZ3-4 & - & 27.4 & - \\
ZZ5-46 & 24.1 & 31.5 & 25.7 & ZZ3-5 & - & 19.4 & - \\
ZZ5-47 & 24.5 & 21.3 & 24.3 & ZZ3-6 & - & 19.7 & - \\
ZZ5-48 & 33.2 & 27.1 & 31.3 & ZZ4-1 & - & 19.7 & - \\
ZZ5-49 & 20.4 & 23.2 & 29.7 & ZZ4-2 & - & 22.9 & - \\
ZZ5-50 & 25.0 & 22.8 & 19.3 & ZZ4-3 & - & 27.9 & - \\
ZZ5-51 & 23.7 & 25.1 & 23.8 & ZZ4-4 & - & 23.2 & - \\
ZZ5-52 & 26.6 & 21.3 & 22.8 & ZZ4-5 & - & 23.8 & - \\
ZZ5-53 & 19.6 & 21.1 & 20.5 & ZZ4-6 & - & 17.0 & - \\
ZZ5-54 & 20.9 & 19.0 & 27.0 & ZZ4-7 & - & 16.1 & - \\
ZZ5-55 & 27.6 & 26.1 & 20.3 & ZZ4-8 & - & 18.5 & - \\
ZZ5-56 & 21.6 & 21.6 & 28.1 & Overall average stiffness & & 24.1 \\
ZZ5-57 & 25.9 & 24.8 & 17.1 & Overall standard deviation & 4.61 \\
ZZ5-58 & 20.3 & 19.9 & 25.2 & Overall COV (\%) & & 19.1 \\
ZZ2-1 & - & 16.4 & - & Overall MAX & & 37.4 \\
ZZ2-2 & - & 25.1 & - & Overall MIN & & 13.1 \\
\hline
\end{tabular}


Table 22. Summary of room-temperature tensile properties for the 3-mm-thick chopped carbon-fiber composite

\begin{tabular}{|c|c|c|c|c|c|}
\hline $\begin{array}{c}\text { Specimen } \\
\text { number }\end{array}$ & UTS (MPa) & $\begin{array}{c}\text { Failure strain } \\
(\%)\end{array}$ & $\begin{array}{c}\text { Specimen } \\
\text { number }\end{array}$ & UTS (MPa) & $\begin{array}{c}\text { Failure strain } \\
(\%)\end{array}$ \\
\hline$\overline{Z 1-1}$ & 183 & 1.11 & Z11-2 & 236 & 1.26 \\
\hline $\mathrm{Z} 1-2$ & 240 & 1.03 & Z11-3 & 177 & 1.21 \\
\hline $\mathrm{Z} 1-3$ & 203 & 0.95 & Z12-5 & 234 & 1.09 \\
\hline Z1-4 & 197 & 0.99 & Z12-16 & 199 & 0.64 \\
\hline$Z 1-5$ & 192 & 1.01 & Z12-22 & 257 & 1.14 \\
\hline Z1-6 & 232 & 0.91 & Z12-36 & 185 & 1.29 \\
\hline $\mathrm{Z} 1-7$ & 201 & 1.00 & Z12-43 & 137 & 0.64 \\
\hline Z1-8 & 206 & 1.00 & Z12-54 & 232 & 0.57 \\
\hline Z1-9 & 243 & 1.01 & Z13-5 & 137 & 0.67 \\
\hline $\mathrm{Z} 1-10$ & 192 & 0.58 & Z13-16 & 190 & 0.99 \\
\hline Z1-11 & 177 & - & Z13-22 & 217 & 0.66 \\
\hline $\mathrm{Z1-12}$ & 209 & 0.69 & Z13-36 & 230 & 0.85 \\
\hline $\mathrm{Z} 2-5$ & 155 & 1.01 & Z13-43 & 222 & 1.01 \\
\hline $\mathrm{Z} 2-16$ & 208 & 0.86 & Z13-54 & 188 & 0.72 \\
\hline $\mathrm{Z} 2-22$ & 191 & 0.77 & Z14-5 & 197 & 0.62 \\
\hline $\mathrm{Z} 2-36$ & 213 & 0.69 & Z14-16 & 194 & 0.90 \\
\hline $\mathrm{Z} 2-54$ & 175 & 0.81 & Z14-22 & 239 & 1.00 \\
\hline Z4-1 & 245 & 1.00 & Z14-36 & 262 & 1.19 \\
\hline Z4-2 & 206 & 0.83 & Z14-43 & 159 & 0.84 \\
\hline Z4-3 & 239 & 0.89 & Z14-54 & 243 & 0.96 \\
\hline Z6-1 & 173 & 0.76 & Z15-1 & 188 & 0.59 \\
\hline Z6-2 & 197 & 0.89 & Z15-2 & 229 & 0.87 \\
\hline Z6-3 & 216 & 1.02 & Z15-3 & 225 & 1.20 \\
\hline Z6-4 & 192 & 0.80 & Z16-5 & 185 & 0.75 \\
\hline$Z 7-5$ & 199 & 0.79 & Z16-16 & 201 & 1.23 \\
\hline Z7-16 & 198 & 0.97 & Z16-43 & 223 & 0.94 \\
\hline$Z 7-43$ & 216 & 1.30 & Z16-54 & 225 & 1.08 \\
\hline$Z 7-54$ & 145 & 0.94 & Z17-1 & 221 & 0.79 \\
\hline Z8-5 & 183 & 1.00 & Z17-2 & 184 & 0.98 \\
\hline Z8-15 & 202 & 0.83 & Z17-3 & 195 & 0.68 \\
\hline Z8-43 & 158 & 0.77 & Z18-1 & 228 & 0.77 \\
\hline Z8-54 & 205 & 0.81 & Z18-2 & 219 & 0.59 \\
\hline Z9-1 & 175 & 1.05 & Z18-3 & 159 & 1.07 \\
\hline Z9-3 & 210 & 0.90 & Z19-1 & 254 & 0.95 \\
\hline Z9-5 & 150 & 1.03 & Z19-2 & 185 & 0.87 \\
\hline Z9-7 & 235 & 1.26 & Z19-3 & 161 & 1.08 \\
\hline Z9-9 & 245 & 1.36 & Z20-5 & 197 & 0.74 \\
\hline Z9-11 & 185 & 1.08 & Z20-16 & 241 & 0.75 \\
\hline Z9-13 & 253 & 0.91 & Z20-22 & 235 & 1.16 \\
\hline Z9-15 & 253 & 0.93 & Z20-36 & 228 & 0.86 \\
\hline Z9-17 & 222 & 0.87 & Z20-43 & 239 & 0.81 \\
\hline Z9-19 & 185 & 0.74 & Z20-54 & 189 & 0.64 \\
\hline Z9-21 & 202 & 1.04 & Z23-1 & 205 & 0.73 \\
\hline Z9-23 & 238 & 1.10 & Z23-2 & 219 & 0.89 \\
\hline Z9-25 & 241 & 0.86 & Z23-3 & 188 & 0.92 \\
\hline Z11-1 & 221 & 0.73 & Z24-A1 & 214 & 0.72 \\
\hline
\end{tabular}

Table 22. Continued. Summary of room-temperature tensile properties for 
the 3-mm-thick chopped carbon-fiber composite

\begin{tabular}{lcc}
\hline $\begin{array}{c}\text { Specimen } \\
\text { number }\end{array}$ & UTS (MPa) & $\begin{array}{c}\text { Failure strain } \\
(\boldsymbol{\%})\end{array}$ \\
\hline Z24-A2 & 136 & 0.67 \\
Z24-B1 & 132 & 0.53 \\
Z24-B2 & 211 & 0.59 \\
Z24-C1 & 201 & 0.97 \\
Z24-C2 & 170 & 0.85 \\
Z24-D1 & 197 & 0.98 \\
Z24-D2 & 180 & 0.79 \\
Z25-A1 & 250 & 0.84 \\
Z25-A2 & 165 & 0.88 \\
Z25-B1 & 222 & 1.13 \\
Z25-B2 & 161 & 0.69 \\
Z25-C1 & 191 & 0.89 \\
Z25-C2 & 185 & 0.90 \\
Z25-D1 & 150 & 0.70 \\
Z25-D2 & 194 & 1.00 \\
Z26-1 & 219 & 0.80 \\
Z26-2 & 232 & 1.08 \\
Z26-3 & 271 & 0.99 \\
Z26-4 & 203 & 0.92 \\
Z26-5 & 190 & 0.96 \\
Z26-6 & 230 & 0.93 \\
Z27-1 & 192 & 0.58 \\
Z27-2 & 212 & 0.90 \\
Z27-3 & 181 & 1.20 \\
Z28-1 & 174 & 0.70 \\
Z28-2 & 241 & 0.88 \\
Z28-3 & 236 & 0.85 \\
Z28-4 & 198 & 0.84 \\
Z28-5 & 177 & 1.18 \\
Z28-6 & 168 & 0.69 \\
Z30-1 & 143 & 1.14 \\
Z30-2 & 161 & 0.86 \\
Z30-3 & 180 & 0.78 \\
Average & 204 & 0.91 \\
SD & 30.8 & 0.18 \\
COV (\%) & 15.1 & 20.0 \\
MAX & 271 & 1.36 \\
MIN & 132 & 0.53 \\
\hline & & \\
\hline
\end{tabular}


Table 23. Summary of room-temperature tensile properties for the 1.5-mm-thick chopped carbon-fiber composite

\begin{tabular}{|c|c|c|c|c|c|c|c|}
\hline $\begin{array}{c}\text { Specimen } \\
\text { Number }\end{array}$ & $\begin{array}{l}\text { UTS } \\
\text { (MPa) }\end{array}$ & $\begin{array}{c}\text { Failure } \\
\text { Strain (\%) }\end{array}$ & $\begin{array}{c}\text { Failure } \\
\text { Location }\end{array}$ & $\begin{array}{l}\text { Specimen } \\
\text { Number }\end{array}$ & $\begin{array}{c}\text { UTS } \\
\text { (MPa) }\end{array}$ & $\begin{array}{c}\text { Failure } \\
\text { Strain (\%) }\end{array}$ & $\begin{array}{c}\text { Failure } \\
\text { Location }\end{array}$ \\
\hline$\overline{Z Z 1-1}$ & 111 & 0.75 & 1 & ZZ3-6 & 179 & 1.32 & - \\
\hline ZZ1-2 & 125 & 0.64 & 1 & ZZ4-1 & 159 & 0.99 & - \\
\hline ZZ1-3 & 160 & 0.99 & 2 & ZZ4-2 & 117 & 0.61 & - \\
\hline ZZ1-4 & 136 & 0.55 & 1 & ZZ4-3 & 153 & 0.74 & - \\
\hline ZZ1-5 & 165 & 1.17 & 2 & ZZ4-4 & 188 & 1.31 & - \\
\hline ZZ1-6 & 92 & 0.46 & 3 & ZZ4-5 & 138 & 1.18 & - \\
\hline ZZ1-7 & 191 & 1.05 & 1 & ZZ4-6 & 123 & 1.17 & - \\
\hline ZZ1-8 & 160 & 0.86 & 2 & ZZ4-7 & 105 & 0.97 & - \\
\hline ZZ1-9 & 174 & 0.57 & 3 & ZZ4-8 & 115 & 0.78 & - \\
\hline ZZ1-10 & 140 & 0.94 & 1 & ZZ5-1 & 120 & 0.55 & 1 \\
\hline ZZ1-11 & 126 & 1.07 & 2 & ZZ5-3 & 196 & 1.01 & 3 \\
\hline ZZ1-12 & 103 & 0.95 & 2 & ZZ5-5 & 176 & 0.92 & 3 \\
\hline ZZ1-13 & 164 & 0.74 & 2 & ZZ5-7 & 217 & 1.23 & 1 \\
\hline ZZ1-14 & 164 & 0.58 & 1 & ZZ5-9 & 141 & 0.70 & 2 \\
\hline ZZ1-15 & 213 & 1.05 & 1 & ZZ5-11 & 124 & 0.68 & 1 \\
\hline ZZ1-16 & 175 & 0.99 & 3 & ZZ5-13 & 132 & 0.61 & 1 \\
\hline ZZ1-17 & 173 & 1.05 & 3 & ZZ5-15 & 163 & 1.48 & 2 \\
\hline ZZ1-18 & 142 & 0.91 & 2 & ZZ5-17 & 173 & 0.67 & 3 \\
\hline ZZ1-19 & 159 & 1.02 & 1 & ZZ5-19 & 157 & 0.68 & 1 \\
\hline ZZ1-20 & 184 & 0.77 & 1 & ZZ5-21 & 178 & 1.23 & 2 \\
\hline ZZ1-21 & 86 & 0.69 & 2 & ZZ5-23 & 176 & 1.03 & 3 \\
\hline ZZ1-22 & 129 & 0.56 & 3 & ZZ5-25 & 166 & 1.12 & 2 \\
\hline ZZ1-23 & 130 & 0.82 & 2 & ZZ5-27 & 161 & 0.65 & 1 \\
\hline ZZ1-24 & 132 & 0.64 & 1 & ZZ5-29 & 177 & 0.84 & 2 \\
\hline ZZ1-25 & 166 & 0.89 & 1 & ZZ5-31 & 126 & 0.65 & 2 \\
\hline ZZ1-39 & 135 & 1.11 & 2 & ZZ5-33 & 119 & 0.57 & 3 \\
\hline ZZ1-40 & 166 & 0.58 & 3 & ZZ5-35 & 180 & 0.68 & 3 \\
\hline ZZ1-41 & 118 & 1.06 & 2 & ZZ5-37 & 114 & 0.49 & 3 \\
\hline ZZ1-42 & 172 & 1.22 & 2 & ZZ5-41 & 128 & 0.67 & 3 \\
\hline ZZ1-43 & 151 & 1.03 & 1 & ZZ5-43 & 163 & 0.99 & 3 \\
\hline ZZ1-54 & 143 & 0.89 & 2 & ZZ5-45 & 165 & 0.67 & 1 \\
\hline ZZ2-1 & 141 & 1.27 & - & ZZ5-47 & 163 & 1.20 & 2 \\
\hline ZZ2-2 & 173 & 0.91 & - & ZZ5-49 & 154 & 0.67 & 1 \\
\hline ZZ2-3 & 139 & 1.23 & - & ZZ5-51 & 182 & 1.14 & 1 \\
\hline ZZ2-4 & 141 & 0.72 & - & ZZ5-53 & 142 & 0.75 & 1 \\
\hline ZZ2-5 & 130 & 0.81 & - & ZZ5-55 & 139 & 0.76 & 3 \\
\hline ZZ2-6 & 209 & 0.85 & - & ZZ5-57 & 149 & 0.68 & 3 \\
\hline ZZ2-7 & 223 & 0.80 & - & & & & \\
\hline ZZ2-8 & 171 & 0.82 & - & Average & 153 & 0.87 & \\
\hline ZZ3-1 & 146 & 0.84 & - & SD & 28.4 & 0.23 & \\
\hline ZZ3-2 & 144 & 0.83 & - & $\operatorname{COV}(\%)$ & 18.6 & 26.5 & \\
\hline ZZ3-3 & 143 & 0.58 & - & MAX & 223 & 1.48 & \\
\hline ZZ3-4 & 198 & 0.87 & - & MIN & 86.4 & 0.46 & \\
\hline ZZ3-5 & 149 & 0.83 & - & & & & \\
\hline
\end{tabular}


Table 24. Summary of plaque average tensile properties the 3-mm-thick chopped-carbonfiber composite

\begin{tabular}{|c|c|c|c|c|c|c|c|c|c|}
\hline $\begin{array}{l}\text { Plaque } \\
\text { number }\end{array}$ & $\begin{array}{c}\text { Number of } \\
\text { stiffness tests }\end{array}$ & $\begin{array}{c}\mathbf{E}_{\text {avg }} \\
\text { (GPa) }\end{array}$ & $\begin{array}{c}\mathbf{E}_{\max } \\
(\mathbf{G P a})\end{array}$ & $\begin{array}{c}\mathbf{E}_{\min } \\
(\mathbf{G P a})\end{array}$ & $\begin{array}{l}\text { Number of } \\
\text { tensile tests }\end{array}$ & $\begin{array}{l}\text { UTS }_{\text {avg }} \\
(\mathrm{MPa})\end{array}$ & $\begin{array}{l}\mathbf{U T S}_{\max } \\
\text { (MPa) }\end{array}$ & $\begin{array}{l}\mathbf{U T S}_{\text {min }} \\
\text { (MPa) }\end{array}$ & $\begin{array}{l}\varepsilon_{\text {f avg }} \\
(\%)\end{array}$ \\
\hline$\overline{\mathrm{Z1}}$ & 12 & 27.3 & 36.1 & 21.5 & 12 & 206 & 243 & 177 & 0.94 \\
\hline $\mathrm{Z2}$ & 58 & 26.4 & 33.7 & 18.0 & 5 & 188 & 213 & 155 & 0.83 \\
\hline Z4 & 3 & 29.9 & 30.5 & 29.5 & 3 & 230 & 245 & 206 & 0.91 \\
\hline Z6 & 4 & 28.8 & 34.2 & 25.1 & 4 & 195 & 216 & 173 & 0.87 \\
\hline Z7 & 58 & 28.8 & 39.6 & 17.9 & 4 & 190 & 216 & 145 & 1.00 \\
\hline Z8 & 58 & 24.6 & 33.7 & 16.7 & 4 & 187 & 205 & 158 & 0.85 \\
\hline Z9 & 26 & 27.4 & 35.1 & 19.0 & 13 & 215 & 253 & 150 & 1.01 \\
\hline Z11 & 3 & 25.6 & 32.1 & 21.7 & 3 & 211 & 236 & 177 & 1.07 \\
\hline Z12 & 57 & 27.5 & 36.7 & 17.7 & 6 & 208 & 257 & 137 & 0.90 \\
\hline Z13 & 54 & 27.3 & 33.9 & 20.6 & 6 & 197 & 230 & 137 & 0.82 \\
\hline Z14 & 53 & 29.2 & 44.7 & 16.4 & 6 & 216 & 262 & 159 & 0.92 \\
\hline Z15 & 3 & 31.8 & 38.0 & 26.3 & 3 & 214 & 229 & 188 & 0.59 \\
\hline Z16 & 58 & 28.0 & 39.6 & 19.1 & 4 & 209 & 225 & 185 & 1.00 \\
\hline Z17 & 3 & 28.5 & 30.1 & 27.0 & 3 & 200 & 221 & 184 & 0.81 \\
\hline Z18 & 3 & 32.3 & 42.8 & 23.4 & 3 & 202 & 228 & 159 & 0.81 \\
\hline Z19 & 3 & 29.4 & 32.2 & 27.9 & 3 & 200 & 254 & 161 & 0.97 \\
\hline Z20 & 58 & 29.9 & 39.6 & 20.9 & 6 & 221 & 241 & 189 & 0.82 \\
\hline Z23 & 3 & 27.7 & 29.4 & 24.3 & 3 & 204 & 219 & 188 & 0.85 \\
\hline $\mathrm{Z} 24$ & 8 & 27.3 & 37.9 & 22.9 & 8 & 180 & 215 & 132 & 0.76 \\
\hline Z25 & 8 & 27.0 & 33.8 & 24.3 & 8 & 190 & 250 & 150 & 0.88 \\
\hline Z26 & 6 & 28.1 & 32.2 & 23.6 & 6 & 224 & 271 & 190 & 0.95 \\
\hline Z27 & 3 & 30.4 & 39.6 & 24.0 & 3 & 195 & 212 & 181 & 0.89 \\
\hline Z28 & 6 & 29.4 & 36.3 & 20.4 & 6 & 199 & 241 & 168 & 0.86 \\
\hline Z30 & 3 & 23.2 & 28.0 & 19.4 & 3 & 162 & 180 & 143 & 0.93 \\
\hline $\begin{array}{l}\text { Number } \\
\text { of tests }\end{array}$ & 551 & & & & 125 & & & & \\
\hline Average & & 27.7 & & & & 203 & & & 0.91 \\
\hline SD & & 4.49 & & & & 30.4 & & & 0.18 \\
\hline $\operatorname{COV} \%$ & & 16.2 & & & & 15.0 & & & 20.1 \\
\hline MAX & & 44.7 & & & & 271 & & & 1.36 \\
\hline MIN & & 16.4 & & & & 132 & & & 0.53 \\
\hline
\end{tabular}


Table 25. Summary of plaque average tensile properties for the 1.5-mm-thick choppedcarbon-fiber composite

\begin{tabular}{|c|c|c|c|c|c|c|c|c|c|}
\hline $\begin{array}{l}\text { Plaque } \\
\text { number }\end{array}$ & $\begin{array}{c}\text { Number of } \\
\text { stiffness tests }\end{array}$ & $\begin{array}{c}\mathbf{E}_{\text {avg }} \\
\text { (GPa) }\end{array}$ & $\begin{array}{c}\mathbf{E}_{\max } \\
(\mathbf{G P a})\end{array}$ & $\begin{array}{c}E_{\min } \\
(\mathrm{GPa})\end{array}$ & $\begin{array}{l}\text { Number of } \\
\text { tensile tests }\end{array}$ & $\begin{array}{l}\text { UTS }_{\text {avg }} \\
(\mathrm{MPa})\end{array}$ & $\begin{array}{l}\mathbf{U T S}_{\max } \\
(\mathrm{MPa})\end{array}$ & $\begin{array}{l}\mathrm{UTS}_{\min } \\
(\mathrm{MPa})\end{array}$ & $\begin{array}{l}\varepsilon_{\mathrm{f} \text { avg }} \\
(\%)\end{array}$ \\
\hline$\overline{Z Z 1}$ & 174 & 23.4 & 37.4 & 13.1 & 31 & 147 & 213 & 86.1 & 0.86 \\
\hline ZZ2 & 8 & 23.8 & 35.1 & 16.4 & 8 & 166 & 223 & 130 & 0.93 \\
\hline ZZ3 & 6 & 23.1 & 27.4 & 19.4 & 6 & 160 & 198 & 143 & 0.88 \\
\hline ZZ4 & 8 & 21.1 & 27.9 & 16.1 & 8 & 137 & 188 & 105 & 0.97 \\
\hline ZZ5 & 171 & 25.1 & 36.3 & 17.1 & 28 & 157 & 217 & 114 & 0.81 \\
\hline $\begin{array}{l}\text { Number } \\
\text { of tests }\end{array}$ & 367 & & & & 81 & & & & \\
\hline Average & & 24.1 & & & & 153 & & & 0.87 \\
\hline SD & & 4.60 & & & & 28.4 & & & 0.23 \\
\hline $\mathrm{COV} \%$ & & 19.1 & & & & 18.6 & & & 26.5 \\
\hline MAX & & 37.4 & & & & 223 & & & 1.48 \\
\hline MIN & & 13.1 & & & & 86.4 & & & 0.46 \\
\hline
\end{tabular}


Table 26. Summary of tensile results for specimens of various widths for the 3-mm-thick chopped-carbon-fiber composite

\begin{tabular}{|c|c|c|c|}
\hline $\begin{array}{c}\text { Specimen } \\
\text { number }\end{array}$ & $\begin{array}{c}\text { Stiffness } \\
(\mathrm{GPa})\end{array}$ & $\begin{array}{c}\text { UTS } \\
\text { (MPa) }\end{array}$ & $\begin{array}{c}\text { Failure } \\
\text { strain }(\%) \\
\end{array}$ \\
\hline \multicolumn{4}{|c|}{ Width $=10.2 \mathrm{~mm}$} \\
\hline $\mathrm{Z} 1-1 \mathrm{~N}$ & 29.5 & 218 & 1.03 \\
\hline $\mathrm{Z} 1-2 \mathrm{~N}$ & 18.8 & 137 & 0.81 \\
\hline $\mathrm{Z} 1-3 \mathrm{~N}$ & 23.0 & 169 & 0.79 \\
\hline $\mathrm{Z} 1-4 \mathrm{~N}$ & 23.0 & 191 & 1.12 \\
\hline Average & 23.6 & 179 & 0.94 \\
\hline SD & 4.42 & 34.2 & 0.16 \\
\hline $\operatorname{COV}(\%)$ & 18.7 & 19.1 & 17.4 \\
\hline \multicolumn{4}{|c|}{ Width $=20.3 \mathrm{~mm}$} \\
\hline Z1-1 & 22.1 & 183 & 1.11 \\
\hline $\mathrm{Z} 1-2$ & 27.7 & 240 & 1.03 \\
\hline Z1-3 & 24.5 & 203 & 0.95 \\
\hline Z1-4 & 24.1 & 197 & 0.99 \\
\hline Average & 24.6 & 206 & 1.02 \\
\hline SD & 2.32 & 24.3 & 0.07 \\
\hline $\operatorname{COV}(\%)$ & 9.42 & 11.8 & 6.56 \\
\hline \multicolumn{4}{|c|}{ Width $=40.6 \mathrm{~mm}$} \\
\hline Z1-1W & 30.2 & 217 & 0.76 \\
\hline $\mathrm{Z} 1-2 \mathrm{~W}$ & 27.0 & 277 & 1.06 \\
\hline Z1-3W & 27.7 & 214 & 0.70 \\
\hline Z1-4W & 31.0 & 237 & 0.84 \\
\hline Average & 29.0 & 236 & 0.84 \\
\hline SD & 1.92 & 28.8 & 0.16 \\
\hline $\operatorname{COV}(\%)$ & 6.64 & 12.2 & 18.7 \\
\hline \multicolumn{4}{|c|}{ Width $=73.5 \mathrm{~mm}$} \\
\hline Z4-3 & - & 256 & - \\
\hline Z4-4 & - & 273 & - \\
\hline Z11-3 & - & 231 & - \\
\hline Z11-4 & - & 257 & - \\
\hline Z15-3 & - & 266 & - \\
\hline Z15-4 & - & 225 & - \\
\hline Z17-3 & - & 231 & - \\
\hline Z17-4 & - & 282 & - \\
\hline Z18-3 & - & 266 & - \\
\hline Z18-4 & - & 250 & - \\
\hline Z19-3 & - & 246 & - \\
\hline Z19-4 & - & 249 & - \\
\hline Average & - & 253 & - \\
\hline SD & - & 17.7 & - \\
\hline $\operatorname{COV}(\%)$ & - & 6.99 & - \\
\hline
\end{tabular}


Table 27. Summary of tensile tests at various strain rates for the 3-mm-thick chopped carbon-fiber composite

\begin{tabular}{|c|c|c|c|c|}
\hline $\begin{array}{c}\begin{array}{c}\text { Specimen } \\
\text { number }\end{array} \\
\end{array}$ & $\begin{array}{c}\text { Virgin stiffness } \\
(\text { GPa })\end{array}$ & $\begin{array}{c}\text { Stiffness } \\
(\mathrm{GPa})\end{array}$ & $\begin{array}{c}\text { UTS } \\
\text { (MPa) }\end{array}$ & $\begin{array}{c}\text { Failure strain } \\
(\%)\end{array}$ \\
\hline \multicolumn{5}{|c|}{ Strain rate $=10^{-6} \mathrm{~s}^{-1}$} \\
\hline Z12-6 & 31.4 & 29.2 & 230 & 0.80 \\
\hline Z12-17 & 33.2 & 32.7 & 218 & 0.73 \\
\hline Z12-30 & 25.4 & 25.4 & 196 & 0.96 \\
\hline Z12-55 & 27.4 & 28.3 & 223 & 0.85 \\
\hline Average & 29.3 & 28.9 & 217 & 0.84 \\
\hline SD & 3.56 & 3.01 & 14.7 & 0.10 \\
\hline $\operatorname{COV}(\%)$ & 12.1 & 10.4 & 6.80 & 11.6 \\
\hline \multicolumn{5}{|c|}{ Strain rate $=10^{-4} \mathrm{~s}^{-1}$} \\
\hline $\mathrm{Z} 12-7$ & 25.5 & 24.0 & 199 & 0.88 \\
\hline Z12-18 & 30.6 & 28.7 & 235 & 0.62 \\
\hline Z12-31 & 31.8 & 35.1 & 197 & 0.56 \\
\hline Z12-40 & 19.9 & 17.9 & 219 & 1.05 \\
\hline Average & 27.0 & 26.4 & 212 & 0.78 \\
\hline SD & 5.43 & 7.29 & 18.0 & 0.23 \\
\hline $\operatorname{COV}(\%)$ & 20.1 & 27.6 & 8.50 & 29.4 \\
\hline \multicolumn{5}{|c|}{ Strain rate $=10^{-2} \mathrm{~s}^{-1}$} \\
\hline Z12-8 & 27.9 & 28.1 & 219 & 0.86 \\
\hline Z12-19 & 25.6 & 25.8 & 217 & 0.90 \\
\hline Z12-32 & 33.4 & 33.1 & 209 & 0.91 \\
\hline Z12-41 & 24.1 & 25.9 & 216 & 0.99 \\
\hline Average & 27.7 & 28.2 & 215 & 0.91 \\
\hline SD & 4.06 & 3.43 & 4.52 & 0.05 \\
\hline $\operatorname{COV}(\%)$ & 14.6 & 12.2 & 2.10 & 5.92 \\
\hline \multicolumn{5}{|c|}{ Strain rate $=10 \mathrm{~s}^{-1}$} \\
\hline Z12-9 & 24.9 & 16.1 & 229 & 1.24 \\
\hline Z12-20 & 28.2 & 21.0 & 170 & 0.80 \\
\hline Z12-33 & 33.1 & 23.4 & 182 & 0.46 \\
\hline Z12-42 & 17.7 & 13.7 & 128 & 0.99 \\
\hline Average & 26.0 & 18.6 & 177 & 0.87 \\
\hline SD & 6.48 & 4.42 & 41.7 & 0.33 \\
\hline $\operatorname{COV}(\%)$ & 25.0 & 23.8 & 23.5 & 37.7 \\
\hline
\end{tabular}


Table 28. Summary of tensile tests at various temperatures for the 3-mm-thick chopped carbon-fiber composite

\begin{tabular}{|c|c|c|c|c|c|c|}
\hline $\begin{array}{c}\text { Specimen } \\
\text { number }\end{array}$ & $\begin{array}{c}\text { Virgin stiffness } \\
\text { (GPa) }\end{array}$ & $\begin{array}{l}\text { Stiffness } \\
\text { (GPa) }\end{array}$ & $\begin{array}{l}\text { UTS } \\
\text { (MPa) }\end{array}$ & $\begin{array}{c}\text { Failure strain } \\
(\%)\end{array}$ & $\begin{array}{l}\text { Stiffness } \\
\text { factor }\end{array}$ & $\begin{array}{c}\text { Strength } \\
\text { factor }\end{array}$ \\
\hline & & & $-40^{\circ} \mathrm{C}$ & & & \\
\hline Z20-4 & 31.1 & 32.1 & 223 & 0.99 & 1.03 & 1.01 \\
\hline Z20-15 & 20.9 & 22.8 & 182 & 0.91 & 1.09 & 0.82 \\
\hline Z20-21 & 36.2 & 38.1 & 236 & 0.64 & 1.05 & 1.07 \\
\hline Z20-35 & 26.1 & 28.8 & 180 & 0.69 & 1.10 & 0.81 \\
\hline Z20-42 & 31 & 30.8 & 186 & 0.68 & 1.00 & 0.84 \\
\hline Z20-53 & 27.5 & 26.1 & 190 & 0.85 & 0.95 & 0.86 \\
\hline Average & 28.8 & 29.8 & 199 & 0.79 & 1.04 & 0.90 \\
\hline SD & 5.22 & 5.30 & 24.0 & 0.14 & & \\
\hline \multirow[t]{2}{*}{$\operatorname{COV}(\%)$} & 18.1 & 17.8 & 12.0 & 18.1 & & \\
\hline & & & $-20^{\circ} \mathrm{C}$ & & & \\
\hline Z20-1 & 38.3 & 41.5 & 250 & 0.74 & 1.08 & 1.13 \\
\hline Z20-12 & 28.7 & 30.1 & 230 & 1.25 & 1.05 & 1.04 \\
\hline Z20-27 & 33.2 & 34.5 & 248 & 0.89 & 1.04 & 1.12 \\
\hline Z20-32 & 29.2 & 28.5 & 233 & 1.16 & 0.98 & 1.05 \\
\hline Z20-39 & 27.2 & 27.4 & 231 & 0.97 & 1.01 & 1.04 \\
\hline Z20-50 & 32.1 & 35.4 & 216 & 0.84 & 1.10 & 0.98 \\
\hline Average & 4.56 & 32.9 & 234 & 0.98 & 1.04 & 1.06 \\
\hline SD & 0.58 & 5.29 & 12.5 & 0.20 & & \\
\hline \multirow[t]{2}{*}{$\operatorname{COV}(\%)$} & 12.8 & 16.1 & 5.33 & 20.0 & & \\
\hline & & & $\mathbf{0}^{\circ} \mathrm{C}$ & & & \\
\hline Z20-2 & 25.9 & 29.3 & 187 & 0.63 & 1.13 & 0.84 \\
\hline Z20-13 & 22.5 & 23.6 & 188 & 0.88 & 1.05 & 0.85 \\
\hline Z20-26 & 28.1 & 28.8 & 203 & 0.93 & 1.02 & 0.92 \\
\hline Z20-33 & 34.2 & 34.7 & 236 & 0.85 & 1.02 & 1.07 \\
\hline Z20-40 & 26.9 & 31.0 & 223 & 1.01 & 1.15 & 1.01 \\
\hline Z20-51 & 37.6 & 35.7 & 227 & 0.69 & 0.95 & 1.02 \\
\hline Average & 29.2 & 30.5 & 211 & 0.83 & 1.05 & 0.95 \\
\hline SD & 5.61 & 4.40 & 21.0 & 0.14 & & \\
\hline \multirow[t]{2}{*}{$\operatorname{COV}(\%)$} & 19.2 & 14.4 & 9.95 & 17.4 & & \\
\hline & & & $23^{\circ} \mathrm{C}$ & & & \\
\hline Z20-5 & 32.0 & 32.0 & 197 & 0.74 & 1.00 & 1.00 \\
\hline Z20-16 & 33.2 & 33.2 & 241 & 0.75 & 1.00 & 1.00 \\
\hline Z20-22 & 27.7 & 27.7 & 235 & 1.16 & 1.00 & 1.00 \\
\hline Z20-36 & 32.1 & 32.1 & 228 & 0.86 & 1.00 & 1.00 \\
\hline Z20-43 & 35.0 & 35.0 & 239 & 0.81 & 1.00 & 1.00 \\
\hline Z20-54 & 34.5 & 34.5 & 189 & 0.64 & 1.00 & 1.00 \\
\hline Average & 32.4 & 32.4 & 221 & 0.82 & 1.00 & 1.00 \\
\hline SD & 2.60 & 2.60 & 22.6 & 0.18 & & \\
\hline $\operatorname{COV}(\%)$ & 8.02 & 8.02 & 10.2 & 21.9 & & \\
\hline
\end{tabular}


Table 28. Continued. Summary of tensile tests at various temperatures for the 3-mm-thick chopped carbon-fiber composite

\begin{tabular}{|c|c|c|c|c|c|c|}
\hline $\begin{array}{c}\text { Specimen } \\
\text { number }\end{array}$ & $\begin{array}{c}\text { Virgin stiffness } \\
\text { (GPa) }\end{array}$ & $\begin{array}{l}\text { Stiffness } \\
\text { (GPa) }\end{array}$ & $\begin{array}{l}\text { UTS } \\
\text { (MPa) }\end{array}$ & $\begin{array}{c}\text { Failure strain } \\
(\%)\end{array}$ & $\begin{array}{l}\text { Stiffness } \\
\text { factor }\end{array}$ & $\begin{array}{l}\text { Strength } \\
\text { factor }\end{array}$ \\
\hline \multicolumn{7}{|c|}{$50^{\circ} \mathrm{C}$} \\
\hline Z20-8 & 24.5 & 23.7 & 187 & 0.95 & 0.97 & 0.84 \\
\hline Z20-19 & 34.4 & 33.4 & 192 & 0.85 & 0.97 & 0.87 \\
\hline Z20-28 & 24.7 & 22.2 & 174 & 0.86 & 0.90 & 0.79 \\
\hline Z20-31 & 39.6 & 37.5 & 216 & 0.66 & 0.95 & 0.98 \\
\hline Z20-46 & 29.6 & 28.5 & 245 & 1.15 & 0.96 & 1.11 \\
\hline Z20-57 & 34.0 & 33.8 & 171 & 0.56 & 0.99 & 0.77 \\
\hline Average & 31.1 & 29.9 & 198 & 0.84 & 0.96 & 0.89 \\
\hline SD & 5.99 & 6.08 & 28.4 & 0.21 & & \\
\hline $\operatorname{COV}(\%)$ & 19.2 & 20.4 & 14.4 & 25.0 & & \\
\hline \multicolumn{7}{|c|}{${ }^{70} 0^{\circ} \mathrm{C}$} \\
\hline Z20-6 & 27.9 & 25.1 & 141 & 0.63 & 0.90 & 0.64 \\
\hline Z20-17 & 31.2 & 31.1 & 199 & 0.60 & 1.00 & 0.90 \\
\hline Z20-23 & 31.9 & 27.3 & 211 & 1.07 & 0.86 & 0.95 \\
\hline Z20-37 & 32.9 & 31.9 & 245 & 1.12 & 0.97 & 1.11 \\
\hline Z20-44 & 28.1 & 24.4 & 183 & 1.01 & 0.87 & 0.83 \\
\hline Z20-55 & 26.3 & 22.2 & 168 & 1.03 & 0.84 & 0.76 \\
\hline Average & 29.7 & 27.0 & 191 & 0.91 & 0.91 & 0.86 \\
\hline SD & 2.62 & 3.84 & 35.9 & 0.23 & & \\
\hline $\operatorname{COV}(\%)$ & 8.83 & 14.2 & 18.8 & 25.5 & & \\
\hline \multicolumn{7}{|c|}{$120^{\circ} \mathrm{C}$} \\
\hline Z20-7 & 26.6 & 21.2 & 144 & 1.00 & 0.80 & 0.65 \\
\hline Z20-18 & 30.4 & 21.9 & 156 & 0.96 & 0.72 & 0.70 \\
\hline Z20-24 & 29.4 & 25 & 162 & 0.65 & 0.85 & 0.73 \\
\hline Z20-38 & 23.6 & 22.3 & 148 & 0.89 & 0.95 & 0.67 \\
\hline Z20-45 & 31.9 & 27.2 & 144 & 0.74 & 0.85 & 0.65 \\
\hline Z20-56 & 26.7 & 20.1 & 135 & 1.12 & 0.75 & 0.61 \\
\hline Average & 28.1 & 22.9 & 148 & 0.89 & 0.82 & 0.67 \\
\hline SD & 3.03 & 2.65 & 9.52 & 0.17 & & \\
\hline $\operatorname{COV}(\%)$ & 10.8 & 11.5 & 6.42 & 19.3 & & \\
\hline
\end{tabular}


Table 29. Temperature multiplication factors for determining at-temperature tensile modulus and strength from room-temperature values for the 3-mm-thick chopped-carbonfiber composite

\begin{tabular}{ccc}
\hline $\begin{array}{c}\text { Temperature } \\
\left({ }^{\circ} \mathbf{C}\right)\end{array}$ & $\begin{array}{c}\text { Stiffness multiplication } \\
\text { factor }\end{array}$ & $\begin{array}{c}\text { Strength multiplication } \\
\text { factor }\end{array}$ \\
\hline $\mathbf{- 4 0}$ & 1.05 & 0.98 \\
$\mathbf{- 2 0}$ & 1.04 & 1.00 \\
$\mathbf{0}$ & 1.03 & 1.01 \\
$\mathbf{2 3}$ & 1.00 & 1.00 \\
$\mathbf{5 0}$ & 0.96 & 0.95 \\
$\mathbf{7 0}$ & 0.93 & 0.90 \\
$\mathbf{1 2 0}$ & 0.81 & 0.69 \\
\hline
\end{tabular}

Table 30. Summary of Poisson's ratio measurements at different temperatures for the 3mm-thick chopped carbon-fiber composite

\begin{tabular}{lcccc}
\hline Specimen number & \multicolumn{4}{c}{ Poisson's ratio } \\
\cline { 2 - 5 } & $\mathbf{- 4 0 ^ { \circ } \mathbf { C }}$ & $\mathbf{2 3}^{\circ} \mathbf{C}$ & $\mathbf{7 0}^{\circ} \mathbf{C}$ & $\mathbf{1 2 0}^{\circ} \mathbf{C}$ \\
\hline Z20-9 & 0.24 & 0.26 & 0.30 & 0.33 \\
Z20-11 & 0.42 & 0.35 & 0.38 & 0.35 \\
Z20-20 & 0.52 & 0.50 & 0.47 & 0.43 \\
Z20-30 & 0.29 & 0.26 & 0.25 & 0.22 \\
Z20-47 & 0.30 & 0.27 & 0.30 & 0.30 \\
Z20-58 & 0.30 & 0.34 & 0.33 & 0.37 \\
& & & & \\
Average & 0.35 & 0.33 & 0.34 & 0.33 \\
SD & 0.10 & 0.09 & 0.08 & 0.07 \\
COV (\%) & 30.2 & 28.0 & 22.8 & 21.2 \\
\hline
\end{tabular}


Table 31. Effects of prior thermal cycling on mechanical properties of the 3-mm-thick chopped carbon-fiber composite

\begin{tabular}{|c|c|c|c|c|c|c|}
\hline $\begin{array}{c}\text { Specimen } \\
\text { number }\end{array}$ & $\begin{array}{c}\text { Virgin Stiffness } \\
\text { (GPa) }\end{array}$ & $\begin{array}{c}\text { Stiffness } \\
(\mathrm{GPa})\end{array}$ & $\begin{array}{l}\text { Strength } \\
\text { (MPa) }\end{array}$ & $\begin{array}{c}\text { Poisson's } \\
\text { ratio }\end{array}$ & $\begin{array}{c}\text { Stiffness } \\
\text { factor }\end{array}$ & $\begin{array}{c}\begin{array}{c}\text { Strength } \\
\text { factor }\end{array} \\
\end{array}$ \\
\hline \multicolumn{7}{|c|}{ Tension } \\
\hline Z12-10 & 26.1 & 27.0 & 205 & - & 1.03 & 0.99 \\
\hline Z12-15 & 26.3 & 23.9 & 190 & 0.29 & 0.91 & 0.91 \\
\hline Z12-21 & 29.3 & 27.2 & 174 & 0.27 & 0.93 & 0.84 \\
\hline Z12-25 & 27.8 & 28.1 & 204 & 0.34 & 1.01 & 0.98 \\
\hline Z12-48 & 29.6 & 30.8 & 233 & 0.35 & 1.04 & 1.12 \\
\hline Z12-56 & 27.1 & 26.8 & 206 & 0.34 & 0.99 & 0.99 \\
\hline Average & 27.7 & 27.3 & 202 & 0.32 & 0.99 & 0.97 \\
\hline SD & 1.51 & 2.20 & 19.5 & & & \\
\hline $\operatorname{COV}(\%)$ & 5.47 & 8.07 & 9.67 & & & \\
\hline \multicolumn{7}{|c|}{ Compression } \\
\hline Z28-9 & - & 21.3 & 184 & - & 0.85 & 1.08 \\
\hline Z28-18 & - & 23.4 & 153 & - & 0.93 & 0.90 \\
\hline Z28-19 & - & 24.5 & 198 & - & 0.98 & 1.16 \\
\hline Z28-31 & - & 29.1 & 113 & - & 1.16 & 0.66 \\
\hline Z28-47 & - & 26.1 & 180 & - & 1.04 & 1.05 \\
\hline Z28-52 & - & 21.9 & 145 & - & 0.87 & 0.85 \\
\hline Average & - & 24.4 & 162 & - & 0.97 & 0.95 \\
\hline SD & - & 2.88 & 31.1 & - & & \\
\hline $\operatorname{COV}(\%)$ & - & 11.8 & 19.2 & - & & \\
\hline \multicolumn{7}{|c|}{ Shear } \\
\hline Z28-5 & - & 8.34 & 123 & - & 0.80 & 0.82 \\
\hline Z28-8 & - & 8.41 & 145 & - & 0.81 & 0.97 \\
\hline Z28-10 & - & 9.51 & 152 & - & 0.91 & 1.01 \\
\hline Z28-31 & - & 11.6 & 134 & - & 1.11 & 0.90 \\
\hline Z28-33 & - & 8.48 & 134 & - & 0.81 & 0.89 \\
\hline Average & - & 9.27 & 138 & - & 0.89 & 0.92 \\
\hline SD & - & 1.38 & 11.0 & - & & \\
\hline $\operatorname{COV}(\%)$ & - & 14.9 & 8.00 & - & & \\
\hline
\end{tabular}


Table 32. Effects of prior cooling to $-40^{\circ} \mathrm{C}$ on tensile properties of the 3-mm-thick chopped carbon-fiber composite

\begin{tabular}{lcccccc}
\hline $\begin{array}{c}\text { Specimen } \\
\text { number }\end{array}$ & $\begin{array}{c}\text { Virgin Stiffness } \\
(\mathbf{G P a})\end{array}$ & $\begin{array}{c}\text { Stiffness } \\
\mathbf{( G P a )}\end{array}$ & $\begin{array}{c}\text { UTS } \\
(\mathbf{M P a})\end{array}$ & $\begin{array}{c}\text { Failure } \\
\text { strain }(\%)\end{array}$ & $\begin{array}{c}\text { Stiffness } \\
\text { factor }\end{array}$ & $\begin{array}{c}\text { Strength } \\
\text { factor }\end{array}$ \\
\hline Z20-3 & 30.7 & 29.6 & 221 & 0.96 & 0.96 & 1.00 \\
Z20-14 & 31.1 & 33.3 & 214 & 0.75 & 1.07 & 0.97 \\
Z20-25 & 27.2 & 29.2 & 228 & 0.97 & 1.08 & 1.03 \\
Z20-34 & 28.1 & 35.2 & 279 & 1.05 & 1.26 & 1.26 \\
Z20-41 & 24.3 & 23.0 & 206 & 1.19 & 0.95 & 0.93 \\
Z20-52 & 30.1 & 30.1 & 224 & 0.92 & 1.00 & 1.01 \\
& & & & & & \\
Average & 28.6 & 30.1 & 229 & 0.97 & 1.05 & 1.03 \\
SD & 2.58 & 4.19 & 26.0 & 0.15 & & \\
COV (\%) & 9.02 & 13.9 & 11.4 & 14.9 & & \\
\hline
\end{tabular}


Table 33. Effect of exposure in $23^{\circ} \mathrm{C}$ distilled water on tensile strength and stiffness of the 3mm-thick chopped-carbon-fiber composite

\begin{tabular}{|c|c|c|c|c|c|c|}
\hline $\begin{array}{c}\text { Specimen } \\
\text { number }\end{array}$ & $\begin{array}{c}\text { Virgin Stiffness } \\
\text { (GPa) }\end{array}$ & $\begin{array}{c}\text { Stiffness } \\
\text { (GPa) }\end{array}$ & $\begin{array}{c}\text { UTS } \\
(\mathrm{MPa})\end{array}$ & $\begin{array}{c}\text { Failure } \\
\text { strain }(\%)\end{array}$ & $\begin{array}{c}\text { Change in } \\
\text { stiffness (\%) }\end{array}$ & $\begin{array}{c}\text { Change in } \\
\text { strength (\%) }\end{array}$ \\
\hline \multicolumn{7}{|c|}{ Exposure Time $=477 \mathrm{~h}$} \\
\hline Z13-4 & 26.1 & 24.6 & 212 & 1.08 & -5.56 & 7.69 \\
\hline Z13-15 & 26.2 & 25.0 & 233 & 1.25 & -4.74 & 18.2 \\
\hline Z13-21 & 33.9 & 31.1 & 246 & 0.94 & -8.15 & 24.8 \\
\hline Z13-33 & 29.5 & 26.3 & 197 & 1.06 & -11.0 & 0.00 \\
\hline Z13-44 & 27.0 & 26.1 & 202 & 0.90 & -3.32 & 2.45 \\
\hline Z13-48 & 21.9 & 20.9 & 141 & 0.75 & -4.72 & -28.3 \\
\hline Average & 27.4 & 25.6 & 205 & 1.00 & -6.24 & 4.14 \\
\hline SD & 3.99 & 3.30 & 36.5 & 0.17 & & \\
\hline $\operatorname{COV}(\%)$ & 14.5 & 12.9 & 17.8 & 17.5 & & \\
\hline \multicolumn{7}{|c|}{ Exposure Time $=1000 \mathrm{~h}$} \\
\hline Z2-4 & 21.0 & 21.0 & 172 & 1.16 & -0.33 & -8.58 \\
\hline Z2-11 & 27.3 & 28.2 & 186 & 0.74 & 3.28 & -1.00 \\
\hline Z2-29 & 31.9 & 31.9 & 201 & 0.70 & 0.00 & 6.70 \\
\hline $\mathrm{Z} 2-38$ & 27.2 & 27.4 & 229 & 0.98 & 0.76 & 21.8 \\
\hline Z2-39 & 22.3 & 23.0 & $a$ & $a$ & 3.41 & - \\
\hline Z2-49 & 20.9 & 21.9 & 166 & 0.96 & 4.95 & -12.0 \\
\hline Z9-2 & 21.0 & 20.7 & 150 & 0.83 & -1.32 & -30.1 \\
\hline Z9-4 & 25.5 & 25.9 & 206 & 1.00 & 1.62 & -4.17 \\
\hline Z9-6 & 35.1 & 33.5 & 215 & 0.80 & -4.52 & 0.00 \\
\hline Z9-8 & 31.5 & 31.3 & 223 & 0.76 & -0.66 & 3.85 \\
\hline Z9-10 & 24.5 & 27.0 & 151 & 0.62 & 10.1 & -30.1 \\
\hline Z9-12 & 25.9 & 26.4 & 195 & 0.79 & 1.86 & -9.62 \\
\hline Z9-14 & 29.0 & 28.5 & 184 & 0.94 & -1.66 & -14.1 \\
\hline Z9-16 & 33.6 & 33.9 & 198 & 0.88 & 0.61 & -8.01 \\
\hline Z9-18 & 30.4 & 29.5 & 208 & 0.81 & -2.95 & -3.21 \\
\hline Z9-20 & 33.6 & 29.9 & 242 & 1.05 & -11.3 & 12.5 \\
\hline Z9-22 & 23.0 & 22.3 & 181 & 1.03 & -3.00 & -15.7 \\
\hline Z9-24 & 27.2 & 24.7 & 194 & 0.99 & -9.37 & -9.62 \\
\hline Z9-26 & 32.8 & 30.4 & 268 & 1.01 & -7.16 & 24.7 \\
\hline Z13-6 & 29.6 & 29.0 & 202 & 0.77 & -2.10 & 2.45 \\
\hline Z13-17 & 26.2 & 26.0 & 202 & 0.95 & -0.79 & 2.45 \\
\hline Z13-23 & 30.9 & 30.3 & 172 & 0.64 & -2.01 & -12.9 \\
\hline Z13-34 & 23.5 & 23.5 & 157 & 0.75 & 0.00 & -20.6 \\
\hline Z13-42 & 23.0 & 22.8 & 149 & 0.82 & -1.05 & -24.5 \\
\hline Z13-57 & 26.1 & 21.6 & 169 & 1.08 & -17.4 & -14.3 \\
\hline Average & 27.3 & 26.8 & 193 & 0.88 & -1.56 & -6.01 \\
\hline SD & 4.35 & 3.99 & 30.1 & 0.15 & & \\
\hline $\operatorname{COV}(\%)$ & 15.9 & 14.9 & 15.6 & 16.6 & & \\
\hline
\end{tabular}


Table 33. Continued. Effect of exposure in $23^{\circ} \mathrm{C}$ distilled water on tensile strength and stiffness of the 3-mm-thick chopped-carbon-fiber composite

\begin{tabular}{|c|c|c|c|c|c|c|}
\hline $\begin{array}{c}\text { Specimen } \\
\text { number }\end{array}$ & $\begin{array}{c}\text { Virgin Stiffness } \\
\text { (GPa) }\end{array}$ & $\begin{array}{l}\text { Stiffness } \\
\text { (GPa) }\end{array}$ & $\begin{array}{l}\text { UTS } \\
\text { (MPa) }\end{array}$ & $\begin{array}{c}\text { Failure } \\
\text { strain }(\%)\end{array}$ & $\begin{array}{c}\text { Change in } \\
\text { stiffness (\%) }\end{array}$ & $\begin{array}{c}\text { Change in } \\
\text { strength (\%) }\end{array}$ \\
\hline \multicolumn{7}{|c|}{ Exposure Time $=1511 \mathrm{~h}$} \\
\hline Z13-7 & 30.8 & 26.1 & 208 & 0.93 & -15.02 & 5.59 \\
\hline Z13-18 & 33.4 & 32.7 & 188 & 0.96 & -2.27 & -4.90 \\
\hline Z13-24 & 27.6 & 26.8 & 205 & 1.03 & -2.99 & 4.20 \\
\hline Z13-35 & 32.4 & 33.0 & 203 & 0.78 & 1.70 & 3.15 \\
\hline Z13-41 & 27.7 & 26.3 & 182 & 0.78 & -4.98 & -7.69 \\
\hline Z13-56 & 27.8 & 27.9 & 208 & 0.82 & 0.50 & 5.59 \\
\hline Average & 30.4 & 28.8 & 199 & 0.88 & -3.84 & 0.99 \\
\hline SD & 2.65 & 3.17 & 11.4 & 0.10 & & \\
\hline $\operatorname{COV}(\%)$ & 8.73 & 11.0 & 5.7 & 11.9 & & \\
\hline \multicolumn{7}{|c|}{ Exposure Time $=2008 \mathrm{~h}$} \\
\hline Z13-8 & 30.8 & 28.7 & 217 & 0.98 & -6.73 & 10.1 \\
\hline Z13-19 & 26.1 & 28.6 & 198 & 0.87 & 9.50 & 0.35 \\
\hline Z13-25 & 23.4 & 24.8 & 175 & 0.81 & 5.59 & -11.2 \\
\hline Z13-37 & 27.2 & 26.7 & 231 & 1.07 & -2.03 & 17.1 \\
\hline Z13-52 & 26.1 & 27.0 & 183 & 0.70 & 3.17 & -6.99 \\
\hline Z13-55 & 30.3 & 31.2 & 224 & 1.00 & 3.08 & 13.6 \\
\hline Average & 26.7 & 27.8 & 205 & 0.91 & 2.10 & 3.85 \\
\hline SD & 2.64 & 2.20 & 22.8 & 0.14 & & \\
\hline $\operatorname{COV}(\%)$ & 9.89 & 7.92 & 11.1 & 15.0 & & \\
\hline \multicolumn{7}{|c|}{ Exposure Time $=5515 \mathrm{~h}$} \\
\hline Z13-9 & 29.6 & 24.8 & 180 & 0.95 & -16.32 & -8.74 \\
\hline Z13-11 & 24.5 & 23.2 & 187 & 0.82 & -5.07 & -5.24 \\
\hline Z13-20 & 23.0 & 24.1 & 191 & 1.20 & 4.79 & -3.15 \\
\hline Z13-26 & 31.0 & 30.2 & 216 & 0.83 & -2.67 & 9.44 \\
\hline Z13-38 & 23.4 & 24.8 & 205 & 1.23 & 5.90 & 3.85 \\
\hline Z13-53 & 30.0 & 28.3 & 192 & 0.76 & -5.52 & -2.80 \\
\hline Average & 26.3 & 25.9 & 195 & 0.97 & -3.15 & -1.11 \\
\hline SD & 3.73 & 2.73 & 13.0 & 0.20 & & \\
\hline $\operatorname{COV}(\%)$ & 14.2 & 10.5 & 6.68 & 21.1 & & \\
\hline
\end{tabular}


Table 34. Effect of exposure in $70 \%$ relative humidity air on tensile strength and stiffness of 3-mm-thick chopped-carbon-fiber composite

\begin{tabular}{|c|c|c|c|c|c|c|}
\hline $\begin{array}{l}\text { Specimen } \\
\text { number }\end{array}$ & $\begin{array}{c}\text { Virgin Stiffness } \\
\text { (GPa) }\end{array}$ & $\begin{array}{c}\text { Stiffness } \\
\text { (GPa) }\end{array}$ & $\begin{array}{l}\text { UTS } \\
\text { (MPa) }\end{array}$ & $\begin{array}{c}\text { Failure } \\
\text { strain }(\%)\end{array}$ & $\begin{array}{c}\text { Change in } \\
\text { stiffness (\%) }\end{array}$ & $\begin{array}{c}\text { Change in } \\
\text { strength }(\%)\end{array}$ \\
\hline \multicolumn{7}{|c|}{ Exposure Time $=1106 \mathrm{~h}$} \\
\hline Z13-10 & 26.6 & 23.3 & 171 & 1.11 & -12.4 & -13.3 \\
\hline Z13-14 & 32.6 & 25.8 & 190 & 0.76 & -20.9 & -3.50 \\
\hline Z13-27 & 25.6 & 26.7 & 177 & 0.79 & 4.31 & -10.1 \\
\hline Z13-30 & 33.8 & 32.3 & 189 & 0.63 & -4.49 & -4.20 \\
\hline Z13-46 & 24.5 & 26.3 & 199 & 0.84 & 7.30 & 0.70 \\
\hline Z13-49 & 22.4 & 22.7 & 183 & 0.83 & 1.23 & -6.99 \\
\hline Average & 27.6 & 26.2 & 185 & 0.83 & -4.17 & -6.24 \\
\hline SD & 4.58 & 3.41 & 9.87 & 0.16 & & \\
\hline $\operatorname{COV}(\%)$ & 16.6 & 13.0 & 5.34 & 19.1 & & \\
\hline \multicolumn{7}{|c|}{ Exposure Time $=2040 \mathrm{~h}$} \\
\hline Z13-2 & 26.0 & 24.1 & 182 & 0.76 & -7.43 & -7.69 \\
\hline Z13-13 & 24.8 & 27.0 & 214 & 0.90 & 8.61 & 8.74 \\
\hline Z13-28 & 30.1 & 28.7 & 210 & 0.87 & -4.81 & 6.64 \\
\hline Z13-31 & 26.2 & 29.0 & 168 & 0.61 & 10.8 & -14.7 \\
\hline Z13-45 & 20.6 & 20.6 & 191 & 1.01 & 0.00 & -3.15 \\
\hline $\mathrm{Z} 13-50$ & 32.4 & 32.2 & 203 & 0.59 & -0.64 & 2.80 \\
\hline Average & 26.7 & 26.9 & 195 & 0.79 & 1.09 & -1.22 \\
\hline SD & 4.14 & 4.08 & 17.7 & 0.17 & & \\
\hline $\operatorname{COV}(\%)$ & 15.5 & 15.2 & 9.10 & 21.2 & & \\
\hline \multicolumn{7}{|c|}{ Exposure Time $=4290 \mathrm{~h}$} \\
\hline Z13-3 & 30.5 & 30.1 & 256 & 0.97 & -1.36 & 30.1 \\
\hline Z13-12 & 28.4 & 24.3 & 201 & 0.92 & -14.3 & 2.10 \\
\hline Z13-29 & 31.0 & 27.9 & 209 & 1.06 & -10.0 & 5.94 \\
\hline Z13-32 & 30.3 & 33.9 & 199 & 0.65 & 11.8 & 1.05 \\
\hline Z13-47 & 31.6 & 31.2 & 214 & 0.77 & -1.31 & 8.74 \\
\hline Z13-51 & 20.9 & 23.0 & 157 & 0.79 & 9.90 & -20.3 \\
\hline Average & 28.8 & 28.4 & 206 & 0.86 & -0.87 & 4.60 \\
\hline SD & 4.02 & 4.17 & 31.9 & 0.15 & & \\
\hline $\operatorname{COV}(\%)$ & 14.0 & 14.7 & 15.4 & 17.5 & & \\
\hline
\end{tabular}


Table 35. Effect of 1000-h exposure in $23^{\circ} \mathrm{C}$ distilled water on tensile strength and stiffness of 1.5-mm-thick chopped-carbon-fiber composite

\begin{tabular}{|c|c|c|c|c|c|c|}
\hline $\begin{array}{c}\begin{array}{c}\text { Specimen } \\
\text { number }\end{array} \\
\end{array}$ & $\begin{array}{c}\text { Virgin Stiffness } \\
(\mathrm{GPa})\end{array}$ & $\begin{array}{c}\text { Stiffness } \\
(\mathrm{GPa})\end{array}$ & $\begin{array}{c}\text { UTS } \\
\text { (MPa) }\end{array}$ & $\begin{array}{c}\text { Failure } \\
\text { strain }(\%)\end{array}$ & $\begin{array}{c}\text { Change in } \\
\text { stiffness (\%) }\end{array}$ & $\begin{array}{c}\text { Change in } \\
\text { strength (\%) }\end{array}$ \\
\hline$\overline{Z Z 5-2}$ & 21.4 & 23.2 & 169 & 1.10 & 8.71 & 7.83 \\
\hline ZZ5-4 & 20.8 & 20.7 & 159 & 1.77 & -0.33 & 0.99 \\
\hline ZZ5-6 & 20.1 & 20.0 & 156 & 1.27 & -0.68 & -0.63 \\
\hline ZZ5-8 & 26.5 & 24.8 & 166 & 0.82 & -6.49 & 5.64 \\
\hline ZZ5-10 & 23.8 & 23.2 & 169 & 0.89 & -2.32 & 7.44 \\
\hline ZZ5-12 & 26.1 & 26.1 & 185 & 0.81 & 0.26 & 17.9 \\
\hline ZZ5-14 & 25.9 & 26.3 & 176 & 0.77 & 1.87 & 12.1 \\
\hline ZZ5-16 & 30.0 & 29.7 & 184 & 1.01 & -0.92 & 17.1 \\
\hline ZZ5-18 & 30.0 & 31.5 & 194 & 0.78 & 5.06 & 23.9 \\
\hline ZZ5-20 & 21.3 & 20.8 & 176 & 1.17 & -2.59 & 12.3 \\
\hline ZZ5-22 & 26.2 & 25.4 & 145 & 1.00 & -3.16 & -7.51 \\
\hline ZZ5-24 & 25.0 & 26.9 & 155 & 1.13 & 7.76 & -1.10 \\
\hline ZZ5-26 & 23.6 & 22.8 & 143 & 1.45 & -3.79 & -8.75 \\
\hline ZZ5-28 & 32.2 & 32.6 & 182 & 0.64 & 1.28 & 16.0 \\
\hline ZZ5-30 & 27.0 & 25.9 & 190 & 0.92 & -4.41 & 20.9 \\
\hline ZZ5-32 & 28.4 & 23.8 & 183 & 0.84 & -16.4 & 16.3 \\
\hline ZZ5-34 & 27.2 & 24.1 & 161 & 0.77 & -11.1 & 2.83 \\
\hline ZZ5-36 & 21.8 & 21.0 & 140 & 1.12 & -3.48 & -10.8 \\
\hline ZZ5-38 & 21.1 & 20.1 & 139 & 0.96 & -4.90 & -11.2 \\
\hline ZZ5-40 & 20.8 & 19.6 & 142 & 0.82 & -5.65 & -9.73 \\
\hline ZZ5-42 & 20.5 & 20.1 & 169 & 1.52 & -1.56 & 7.51 \\
\hline ZZ5-44 & 19.0 & 18.1 & 115 & 0.71 & -5.07 & -26.4 \\
\hline ZZ5-46 & 31.5 & 29.2 & 160 & 0.58 & -7.22 & 1.91 \\
\hline ZZ5-48 & 27.1 & 28.5 & 180 & 0.85 & 5.07 & 14.8 \\
\hline ZZ5-52 & 21.3 & 21.1 & 150 & 1.07 & -0.97 & -4.15 \\
\hline ZZ5-54 & 19.0 & 20.7 & 135 & 0.63 & 8.97 & -14.3 \\
\hline ZZ5-56 & 21.6 & 23.0 & 125 & 0.64 & 6.71 & -20.3 \\
\hline ZZ5-58 & 19.9 & 20.2 & 145 & 1.19 & 1.38 & -7.70 \\
\hline Average & 24.3 & 23.9 & 160 & 0.97 & -1.24 & 2.21 \\
\hline SD & 3.93 & 3.86 & 20.5 & 0.29 & & \\
\hline $\operatorname{COV}(\%)$ & 16.2 & 16.1 & 12.8 & 29.4 & & \\
\hline
\end{tabular}


Table 36. Effects of 100-h exposure in windshield washer fluid on tensile stiffness and strength of 3-mm-thick chopped-carbon-fiber composite

\begin{tabular}{lcccccc}
\hline $\begin{array}{c}\text { Specimen } \\
\text { number }\end{array}$ & $\begin{array}{c}\text { Virgin stiffness } \\
(\mathbf{G P a})\end{array}$ & $\begin{array}{c}\text { Stiffness } \\
(\mathbf{G P a})\end{array}$ & $\begin{array}{c}\text { UTS } \\
\mathbf{( M P a})\end{array}$ & $\begin{array}{c}\text { Failure } \\
\text { strain }(\%)\end{array}$ & $\begin{array}{c}\text { Stiffness } \\
\text { factor }\end{array}$ & $\begin{array}{c}\text { Strength } \\
\text { factor }\end{array}$ \\
\hline Z14-4 & 43.6 & 39.6 & 297 & 0.92 & 0.91 & 1.38 \\
Z14-6 & 33.1 & 33.5 & 233 & 0.69 & 1.01 & 1.08 \\
Z14-15 & 26.2 & 28.3 & 223 & 0.92 & 1.08 & 1.03 \\
Z14-17 & 23.2 & 23.7 & 179 & 0.79 & 1.02 & 0.83 \\
Z14-23 & 26.8 & 28.1 & 227 & 0.96 & 1.05 & 1.05 \\
Z14-28 & 29.9 & 31.0 & 225 & 0.81 & 1.04 & 1.04 \\
Z14-35 & 32.6 & 35.4 & 169 & 0.47 & 1.09 & 0.78 \\
Z14-37 & 35.1 & 31.9 & 254 & 0.80 & 0.91 & 1.18 \\
Z14-42 & 25.0 & 24.9 & 155 & 0.71 & 1.00 & 0.72 \\
Z14-44 & 16.4 & 16.0 & 143 & 1.02 & 0.98 & 0.66 \\
Z14-53 & 28.8 & 29.0 & 204 & 0.75 & 1.01 & 0.95 \\
Z14-55 & 29.9 & 32.1 & 234 & 0.87 & 1.07 & 1.08 \\
& & & & & & \\
Average & 29.2 & 29.5 & 212 & 0.81 & 1.01 & 0.98 \\
SD & 6.76 & 6.09 & 44.1 & 0.15 & & \\
COV (\%) & 23.1 & 20.7 & 20.8 & 18.2 & & \\
\hline
\end{tabular}


Table 37. Summary of room-temperature compressive tests for the 3-mm-thick choppedcarbon-fiber composite

\begin{tabular}{|c|c|c|c|}
\hline $\begin{array}{c}\text { Specimen } \\
\text { number }\end{array}$ & $\begin{array}{c}\text { Compressive } \\
\text { stiffness (GPa) }\end{array}$ & $\begin{array}{l}\text { UCS } \\
(\mathrm{MPa})\end{array}$ & $\begin{array}{c}\text { Failure strain } \\
(\%)\end{array}$ \\
\hline$\overline{Z 1-1}$ & 31.5 & 138 & 0.47 \\
\hline Z1-3 & 23.9 & 173 & 0.78 \\
\hline$Z 1-5$ & 29.7 & 192 & 0.70 \\
\hline Z1-10 & 24.5 & 186 & 0.88 \\
\hline Z1-12 & 30.8 & 176 & 0.67 \\
\hline Z1-14 & 28.5 & 211 & 0.80 \\
\hline Z4-1 & 32.0 & 110 & 0.40 \\
\hline Z4-2 & 20.6 & 131 & 1.16 \\
\hline Z4-3 & 28.0 & 151 & 0.64 \\
\hline Z11-1 & 27.0 & 242 & 1.21 \\
\hline Z11-2 & 24.1 & 168 & 0.89 \\
\hline Z11-3 & 34.7 & 195 & 0.61 \\
\hline Z23-1 & 42.8 & 223 & 0.55 \\
\hline Z23-2 & 28.7 & 178 & 0.75 \\
\hline Z23-3 & $a$ & 204 & $a$ \\
\hline Z27-2 & 32.3 & 183 & 0.68 \\
\hline $\mathrm{Z} 27-3$ & 40.5 & 188 & 0.44 \\
\hline Z28-5 & 27.1 & 160 & 0.92 \\
\hline Z28-15 & 25.8 & 165 & 0.78 \\
\hline Z28-24 & 38.5 & 225 & 0.68 \\
\hline Z15-1 & 39.0 & 180 & 0.55 \\
\hline Z15-2 & 27.3 & 192 & 0.87 \\
\hline Z15-3 & 39.2 & 107 & 0.26 \\
\hline Z17-1 & 32.6 & 135 & 0.57 \\
\hline Z17-2 & 25.0 & 175 & 0.83 \\
\hline Z17-3 & 32.7 & 196 & 0.91 \\
\hline Z18-1 & 36.7 & 196 & 0.55 \\
\hline Z18-2 & 32.7 & 199 & 1.27 \\
\hline Z18-3 & 25.0 & 109 & 0.46 \\
\hline Z19-1 & 36.7 & 196 & 0.55 \\
\hline Z19-2 & 32.7 & 199 & 1.27 \\
\hline Z19-3 & 25.0 & 109 & 0.46 \\
\hline Z28-28 & 18.8 & 169 & 0.95 \\
\hline Z28-39 & 20.8 & 132 & 0.70 \\
\hline Z28-49 & 27.0 & 149 & 0.84 \\
\hline Average & 30.1 & 173 & 0.74 \\
\hline SD & 6.03 & 34.7 & 0.25 \\
\hline $\operatorname{COV}(\%)$ & 20.1 & 20.1 & 33.7 \\
\hline
\end{tabular}

${ }^{a}$ No strain measurement 
Table 38. Summary of in-air room-temperature compressive properties of the 1.5-mm-thick chopped-carbon-fiber composite

\begin{tabular}{lccc}
\hline $\begin{array}{c}\text { Specimen } \\
\text { number }\end{array}$ & $\begin{array}{c}\text { Compressive stiffness } \\
(\mathbf{G P a})\end{array}$ & UCS (MPa) & $\begin{array}{c}\text { Failure strain } \\
\mathbf{( \% )}\end{array}$ \\
\hline ZZ3-2 & 16.2 & 135 & 0.75 \\
ZZ3-5 & 35.8 & 144 & 0.95 \\
ZZ3-8 & 20.2 & 155 & 0.90 \\
ZZ3-11 & 15.2 & 188 & 0.93 \\
ZZ3-14 & 14.7 & 146 & 1.11 \\
ZZ3-17 & 25.7 & 154 & 0.91 \\
ZZ3-20 & 23.0 & 179 & 0.85 \\
ZZ3-23 & 28.5 & 192 & 0.75 \\
& & & \\
Average & 22.4 & 162 & 0.89 \\
SD & 7.39 & 21.7 & 0.12 \\
COV (\%) & 33.0 & 13.4 & 13.0 \\
\hline
\end{tabular}


Table 39. Compressive properties of the 3-mm-thick chopped-carbon-fiber composite at different temperatures

\begin{tabular}{|c|c|c|c|}
\hline Specimen number & $\begin{array}{c}\text { Compressive Stiffness } \\
\text { (GPa) }\end{array}$ & UCS (MPa) & $\begin{array}{c}\text { Failure strain } \\
(\%)\end{array}$ \\
\hline \multicolumn{4}{|c|}{$70^{\circ} \mathrm{C}$} \\
\hline Z28-6 & 21.4 & 149 & 0.83 \\
\hline Z28-16 & 20.5 & 133 & 0.80 \\
\hline $\mathrm{Z} 28-25$ & 25.2 & 144 & 0.84 \\
\hline Z28-29 & 27.7 & 156 & 0.67 \\
\hline Z28-40 & 15.3 & 123 & 0.85 \\
\hline $\mathrm{Z} 28-50$ & 28.7 & 140 & 0.64 \\
\hline Average & 23.1 & 141 & 0.77 \\
\hline SD & 5.05 & 11.7 & 0.09 \\
\hline $\operatorname{COV}(\%)$ & 21.8 & 8.32 & 12.0 \\
\hline \multicolumn{4}{|c|}{$120^{\circ} \mathrm{C}$} \\
\hline Z28-7 & 19.3 & 85.6 & 0.65 \\
\hline Z28-17 & 20.8 & 96.0 & 0.52 \\
\hline Z28-26 & 22.8 & 58.8 & 0.25 \\
\hline Z28-30 & 28.8 & 103 & 0.42 \\
\hline Z28-41 & 27.5 & 83.3 & 0.35 \\
\hline Z28-51 & 18.6 & 85.5 & 0.86 \\
\hline Average & 23.0 & 85.4 & 0.51 \\
\hline SD & 4.29 & 15.1 & 0.22 \\
\hline $\operatorname{COV}(\%)$ & 18.7 & 17.6 & 43.4 \\
\hline \multicolumn{4}{|c|}{$-40^{\circ} \mathrm{C}$} \\
\hline Z28-4 & $a$ & 205 & $a$ \\
\hline Z28-14 & 26.5 & 263 & 0.89 \\
\hline Z28-23 & 32.9 & 184 & 0.62 \\
\hline Z28-27 & 25.2 & 212 & 0.97 \\
\hline Z28-38 & 29.6 & 229 & 0.79 \\
\hline Z28-48 & 23.9 & 196 & 0.92 \\
\hline Average & 27.6 & 215 & 0.84 \\
\hline SD & 3.63 & 28.0 & 0.14 \\
\hline $\operatorname{COV}(\%)$ & 13.1 & 13.1 & 16.5 \\
\hline
\end{tabular}

${ }^{a}$ No strain measurement

Table 40. Temperature multiplication factors for determining at-temperature compressive stiffness and strength from room-temperature values for the 3-mm-thick chopped-carbonfiber composite

\begin{tabular}{ccc}
\hline $\begin{array}{c}\text { Temperature } \\
\left({ }^{\circ} \mathbf{C}\right)\end{array}$ & $\begin{array}{c}\text { Stiffness multiplication } \\
\text { factor }\end{array}$ & $\begin{array}{c}\text { Strength multiplication } \\
\text { factor }\end{array}$ \\
\hline $\mathbf{- 4 0}$ & 1.07 & 1.23 \\
$\mathbf{2 3}$ & 1.00 & 1.00 \\
$\mathbf{7 0}$ & 0.94 & 0.77 \\
$\mathbf{1 2 0}$ & 0.88 & 0.48 \\
\hline
\end{tabular}


Table 41. Effects of 1000-h exposure in $23^{\circ} \mathrm{C}$ distilled water on room-temperature compressive properties of the 3-mm-thick chopped-carbon-fiber composite

\begin{tabular}{lccccc}
\hline $\begin{array}{c}\text { Specimen } \\
\text { number }\end{array}$ & $\begin{array}{c}\text { Compressive stiffness } \\
\text { (GPa) }\end{array}$ & UCS (MPa) & $\begin{array}{c}\text { Failure strain } \\
\text { (\%) }\end{array}$ & $\begin{array}{c}\text { Stiffness } \\
\text { factor }\end{array}$ & $\begin{array}{c}\text { Strength } \\
\text { factor }\end{array}$ \\
\hline Z28-8 & 22.1 & 164 & 0.99 & 0.88 & 0.96 \\
Z28-20 & $a$ & 160 & $a$ & $a$ & 0.94 \\
Z28-22 & 27.0 & 134 & 0.81 & 1.08 & 0.78 \\
Z28-33 & 22.7 & 169 & 1.17 & 0.90 & 0.99 \\
Z28-42 & 28.6 & 122 & 0.59 & 1.14 & 0.71 \\
Average & 25.1 & 150 & & & \\
SD & 3.19 & 20.6 & 0.89 & 1.00 & 0.88 \\
COV (\%) & 12.7 & 13.7 & 27.9 & & \\
\hline
\end{tabular}

${ }^{a}$ No strain measurement

Table 42. Effects of $1000-\mathrm{h}$ exposure in $23^{\circ} \mathrm{C}$ distilled water on room-temperature compressive properties of the 1.5-mm-thick chopped-carbon-fiber composite

\begin{tabular}{lcccc}
\hline $\begin{array}{c}\text { Specimen } \\
\text { number }\end{array}$ & $\begin{array}{c}\text { Compressive stiffness } \\
(\mathbf{G P a})\end{array}$ & UCS (MPa) & $\begin{array}{c}\text { Stiffness } \\
\text { factor }\end{array}$ & $\begin{array}{c}\text { Strength } \\
\text { factor }\end{array}$ \\
\hline ZZ3-1 & 17.0 & 115 & 0.76 & 0.71 \\
ZZ3-4 & 16.2 & 143 & 0.72 & 0.88 \\
ZZ3-7 & 17.7 & 148 & 0.79 & 0.91 \\
ZZ3-13 & 23.6 & 137 & 1.05 & 0.85 \\
ZZ3-16 & 17.8 & 130 & 0.79 & 0.80 \\
ZZ3-19 & 20.2 & 135 & 0.90 & 0.83 \\
ZZ3-22 & 18.1 & 158 & 0.81 & 0.98 \\
& & & & \\
Average & 18.7 & 138 & 0.83 & 0.85 \\
SD & 2.50 & 13.7 & & \\
COV (\%) & 13.4 & 9.94 & & \\
\hline
\end{tabular}

Table 43. Effects of exposure in windshield washer fluid on room-temperature compressive strength of the 3-mm-thick chopped-carbon-fiber composite

\begin{tabular}{lcc}
\hline Specimen Number & UCS (MPa) & Strength factor \\
\hline Z28-13 & 146 & 0.85 \\
Z28-21 & 159 & 0.93 \\
Z28-32 & 174 & 1.02 \\
Z28-34 & 171 & 1.00 \\
Z28-43 & 164 & 0.96 \\
& & \\
Average & 163 & 0.95 \\
SD & 11.1 & \\
COV (\%) & 6.80 & \\
\hline
\end{tabular}


Table 44. Summary of in-air room-temperature shear properties for the 3-mm-thick chopped-carbon-fiber composite

\begin{tabular}{|c|c|c|c|}
\hline Specimen number & G (GPa) & Shear strength (MPa) & Failure strain (\%) \\
\hline$\overline{\mathrm{Z} 4-1}$ & 7.45 & 145 & 2.38 \\
\hline $\mathrm{Z} 4-2$ & 8.48 & 137 & 2.12 \\
\hline $\mathrm{Z} 4-3$ & 9.40 & 146 & 2.28 \\
\hline Z9-1 & 9.10 & 161 & 1.94 \\
\hline Z9-3 & 9.17 & 168 & 2.62 \\
\hline Z9-7 & 10.7 & 139 & 1.62 \\
\hline Z9-9 & 8.48 & 168 & 2.95 \\
\hline Z9-11 & 13.2 & 177 & 2.26 \\
\hline Z9-17 & 9.45 & 151 & 2.12 \\
\hline Z9-21 & 12.0 & 157 & 2.59 \\
\hline Z9-23 & 9.38 & 150 & 2.27 \\
\hline Z9-27 & 9.03 & 164 & 2.31 \\
\hline Z9-29 & 9.72 & 180 & 2.43 \\
\hline Z9-31 & a & 148 & a \\
\hline Z9-5 & a & 138 & $\mathrm{a}$ \\
\hline Z9-13 & a & 170 & a \\
\hline Z9-15 & a & 167 & a \\
\hline Z9-19 & a & 123 & a \\
\hline Z9-25 & $\mathrm{a}$ & 160 & a \\
\hline Z11-1 & $\mathrm{a}$ & 168 & $\mathrm{a}$ \\
\hline Z11-2 & 7.65 & 176 & 3.51 \\
\hline Z11-3 & 7.38 & 163 & a \\
\hline Z15-1 & 8.27 & 141 & 2.49 \\
\hline Z15-2 & 7.58 & 174 & 2.86 \\
\hline Z15-3 & 7.52 & 157 & 2.56 \\
\hline Z17-1 & 10.0 & 166 & 2.98 \\
\hline Z17-2 & 10.0 & 146 & 2.09 \\
\hline Z17-3 & 7.58 & 166 & 3.03 \\
\hline Z18-1 & 8.41 & 135 & 2.97 \\
\hline Z18-2 & 7.38 & 160 & 3.09 \\
\hline Z18-3 & 10.5 & 141 & a \\
\hline Z19-1 & 8.62 & 154 & 2.54 \\
\hline Z19-2 & 9.16 & 139 & 2.39 \\
\hline Z19-3 & 9.86 & 150 & 1.77 \\
\hline Z23-1 & 9.42 & 147 & 2.26 \\
\hline Z23-2 & 7.94 & 148 & 2.32 \\
\hline Z23-3 & 9.36 & 155 & 2.54 \\
\hline Z27-1 & 11.1 & 125 & 1.75 \\
\hline Z27-2 & 10.9 & 143 & 1.36 \\
\hline Z27-3 & 11.1 & 141 & 1.84 \\
\hline Z28-2 & 10.8 & 140 & 1.96 \\
\hline Z28-3 & 10.9 & 161 & 2.10 \\
\hline Z28-13 & 9.17 & 143 & 1.83 \\
\hline Z28-38 & 10.6 & 145 & 1.86 \\
\hline
\end{tabular}


Table 44. Continued. Summary of in-air room-temperature shear properties for the 3-mm-thick chopped-carbon-fiber composite

\begin{tabular}{lccc}
\hline Specimen number & G (GPa) & Shear strength (MPa) & Failure strain (\%) \\
\hline Z28-18 & $\mathrm{a}$ & 156 & $\mathrm{a}$ \\
Z28-28 & $\mathrm{a}$ & 150 & $\mathrm{a}$ \\
& & & \\
Average & 9.37 & 153 & 2.34 \\
SD & 1.40 & 13.6 & 0.47 \\
COV (\%) & 15.0 & 8.87 & 20.2 \\
\hline
\end{tabular}

${ }^{a}$ No strain measurement

Table 45. Summary of in-air room-temperature shear properties for the 1.5-mm-thick chopped-carbon-fiber composite

\begin{tabular}{lccc}
\hline Specimen number & $\begin{array}{c}\text { Shear modulus } \\
(\mathbf{G P a})\end{array}$ & $\begin{array}{c}\text { Shear strength } \\
(\mathbf{M P a})\end{array}$ & $\begin{array}{c}\text { Failure strain } \\
(\mathbf{\%})\end{array}$ \\
\hline ZZ3-2 & 10.7 & 129 & 1.59 \\
ZZ3-5 & 8.14 & 141 & 2.23 \\
ZZ3-8 & 9.79 & 125 & 1.64 \\
ZZ3-11 & 17.2 & 145 & 1.06 \\
ZZ3-14 & 10.1 & 98.7 & 2.16 \\
ZZ3-17 & 13.2 & 156 & 1.71 \\
ZZ3-20 & 11.6 & 157 & 1.93 \\
ZZ3-23 & 9.72 & 129 & 2.53 \\
& & & \\
Average & 11.3 & 135 & 1.86 \\
SD & 2.80 & 19.1 & 0.46 \\
COV (\%) & 24.8 & 14.1 & 24.6 \\
\hline
\end{tabular}


Table 46. Shear properties of the 3-mm-thick chopped-carbon-fiber composite at different temperatures

\begin{tabular}{|c|c|c|c|}
\hline Specimen number & $\begin{array}{c}\text { Shear modulus } \\
\text { (GPa) }\end{array}$ & $\begin{array}{c}\text { Shear strength } \\
\text { (MPa) }\end{array}$ & $\begin{array}{c}\text { Failure strain } \\
(\%)\end{array}$ \\
\hline \multicolumn{4}{|c|}{$7^{\circ} \mathrm{C}$} \\
\hline Z28-14 & 6.55 & 110 & 2.56 \\
\hline Z28-24 & 8.55 & 107 & 1.96 \\
\hline Z28-19 & 6.48 & 107 & 3.13 \\
\hline Z28-25 & 7.79 & 106 & 1.84 \\
\hline Z28-29 & 8.76 & 107 & 1.37 \\
\hline Average & 7.63 & 107 & 2.17 \\
\hline SD & 1.08 & 1.52 & 0.68 \\
\hline $\operatorname{COV}(\%)$ & 14.1 & 1.41 & 31.5 \\
\hline \multicolumn{4}{|c|}{$120^{\circ} \mathrm{C}$} \\
\hline Z28-4 & 6.63 & 84.4 & 1.37 \\
\hline Z28-11 & 7.79 & 95.0 & 2.79 \\
\hline Z28-20 & 9.24 & 81.8 & 0.92 \\
\hline $\mathrm{Z} 28-30$ & 5.84 & 81.7 & 2.39 \\
\hline Z28-37 & 7.14 & 87.7 & 2.18 \\
\hline Z28-26 & $a$ & 90.9 & $a$ \\
\hline Average & 7.33 & 86.9 & 1.93 \\
\hline SD & 1.29 & 5.32 & 0.77 \\
\hline $\operatorname{COV}(\%)$ & 17.5 & 6.12 & 39.7 \\
\hline \multicolumn{4}{|c|}{$-40^{\circ} \mathrm{C}$} \\
\hline Z28-1 & 9.58 & 170 & 1.82 \\
\hline Z28-12 & 12.8 & 197 & 1.33 \\
\hline $\mathrm{Z} 28-17$ & 9.31 & 189 & 1.93 \\
\hline $\mathrm{Z} 28-23$ & 12.3 & 181 & 1.57 \\
\hline Z28-27 & 8.96 & 162 & 2.53 \\
\hline Average & 10.6 & 180 & 1.84 \\
\hline SD & 1.81 & 14.1 & 0.45 \\
\hline $\operatorname{COV}(\%)$ & 17.1 & 7.84 & 24.6 \\
\hline
\end{tabular}

${ }^{a}$ No strain measurement

Table 47. Temperature multiplication factors for determining at-temperature shear modulus and strength from room-temperature values for the 3-mm-thick chopped-carbonfiber composite

\begin{tabular}{ccc}
\hline $\begin{array}{c}\text { Temperature } \\
\left({ }^{\circ} \mathbf{C}\right)\end{array}$ & $\begin{array}{c}\text { Stiffness multiplication } \\
\text { factor }\end{array}$ & $\begin{array}{c}\text { Strength } \\
\text { multiplication factor }\end{array}$ \\
\hline $\mathbf{- 4 0}$ & 1.10 & 1.25 \\
$\mathbf{2 3}$ & 1.00 & 1.00 \\
$\mathbf{7 0}$ & 0.89 & 0.81 \\
$\mathbf{1 2 0}$ & 0.74 & 0.61 \\
\hline
\end{tabular}


Table 48. Effects of 1000 -h exposure in $23^{\circ} \mathrm{C}$ distilled water on room-temperature shear properties of the 3-mm-thick chopped-carbon-fiber composite

\begin{tabular}{lccccc}
\hline $\begin{array}{c}\text { Specimen } \\
\text { number }\end{array}$ & $\begin{array}{c}\text { Shear modulus } \\
(\mathbf{G P a})\end{array}$ & $\begin{array}{c}\text { Shear strength } \\
(\mathbf{M P a})\end{array}$ & $\begin{array}{c}\text { Failure } \\
\text { strain } \mathbf{( \% )}\end{array}$ & $\begin{array}{c}\text { Stiffness } \\
\text { factor }\end{array}$ & $\begin{array}{c}\text { Strength } \\
\text { factor }\end{array}$ \\
\hline Z9-6 & 8.48 & 151 & 3.41 & 0.85 & 0.96 \\
Z9-8 & 8.00 & 147 & 2.18 & 0.80 & 0.93 \\
Z9-12 & 12.7 & 172 & 1.85 & 1.27 & 1.09 \\
Z9-14 & 11.9 & 185 & 1.76 & 1.19 & 1.17 \\
Z9-16 & 10.5 & 150 & 2.27 & 1.05 & 0.95 \\
Z9-20 & 12.3 & 125 & 1.21 & 1.23 & 0.79 \\
Z9-22 & 10.1 & 158 & 2.74 & 1.01 & 1.00 \\
Z9-26 & 9.93 & 172 & 2.24 & 0.99 & 1.09 \\
Z9-28 & 14.5 & 150 & 1.12 & 1.45 & 0.95 \\
Z9-30 & $a$ & 137 & $a$ & $a$ & 0.87 \\
Z9-32 & $a$ & 170 & $a$ & $a$ & 1.08 \\
Z9-2 & $a$ & 157 & $a$ & $a$ & 0.99 \\
Z9-4 & $a$ & 137 & $a$ & $a$ & 0.87 \\
Z9-10 & $a$ & 163 & $a$ & $a$ & 1.03 \\
Z9-18 & $a$ & 126 & $a$ & $a$ & 0.80 \\
Z9-24 & $a$ & 142 & $a$ & $a$ & 0.90 \\
Z28-6 & $a$ & 135 & 3.10 & $a$ & 0.90 \\
Z28-15 & 6.90 & 115 & 2.75 & 0.66 & 0.77 \\
Z28-21 & 8.20 & 150 & 1.74 & 0.79 & 1.00 \\
Z28-32 & 7.03 & 132 & 2.50 & 0.68 & 0.88 \\
Z28-34 & 6.99 & 131 & 2.65 & 0.67 & 0.88 \\
& & & & & \\
Average & 9.81 & 148 & 2.25 & 0.97 & 0.95 \\
SD & 2.47 & 18.1 & 0.67 & & \\
COV (\%) & 25.2 & 12.2 & 29.7 & & \\
\hline$a$ & & & & &
\end{tabular}

${ }^{a}$ No strain measurement 
Table 49. Effects of exposure $1000-\mathrm{h}$ in $23^{\circ} \mathrm{C}$ distilled water on room-temperature shear properties of the 1.5-mm-thick chopped-carbon-fiber composite

\begin{tabular}{lccccc}
\hline $\begin{array}{c}\text { Specimen } \\
\text { number }\end{array}$ & $\begin{array}{c}\text { Shear modulus } \\
(\mathbf{G P a})\end{array}$ & $\begin{array}{c}\text { Shear strength } \\
\mathbf{( M P a )}\end{array}$ & $\begin{array}{c}\text { Failure } \\
\text { strain }(\%)\end{array}$ & $\begin{array}{c}\text { Stiffness } \\
\text { factor }\end{array}$ & $\begin{array}{c}\text { Strength } \\
\text { factor }\end{array}$ \\
\hline ZZ3-1 & 10.5 & 97.2 & 1.63 & 0.93 & 0.72 \\
ZZ3-4 & 10.6 & 115 & 1.32 & 0.94 & 0.85 \\
ZZ3-7 & 13.0 & 123 & 2.04 & 1.15 & 0.91 \\
ZZ3-10 & 11.2 & 121 & 1.66 & 0.99 & 0.90 \\
ZZ3-13 & 9.93 & 108 & 1.74 & 0.88 & 0.80 \\
ZZ3-16 & 9.03 & 145 & 1.73 & 0.80 & 1.07 \\
ZZ3-19 & 10.9 & 112 & 1.36 & 0.96 & 0.83 \\
ZZ3-22 & 9.38 & 135 & 2.08 & 0.83 & 1.00 \\
Average & 10.6 & 120 & & & \\
SD & 1.22 & 15.3 & 0.27 & & 0.99 \\
COV (\%) & 11.5 & 12.8 & 16.2 & & \\
\hline
\end{tabular}

${ }^{a}$ No strain measurement

Table 50. Effects of 100-h exposure in windshield washer fluid on room-temperature shear strength of the 3-mm-thick chopped-carbon-fiber composite

\begin{tabular}{lcc}
\hline $\begin{array}{c}\text { Specimen } \\
\text { number }\end{array}$ & $\begin{array}{c}\text { Shear strength } \\
\text { (MPa) }\end{array}$ & Strength factor \\
\hline Z28-7 & 173 & 1.16 \\
Z28-9 & 124 & 0.83 \\
Z28-16 & 143 & 0.96 \\
Z28-22 & 158 & 1.06 \\
Z28-35 & 140 & 0.94 \\
& & \\
Average & 148 & 0.99 \\
SD & 18.4 & \\
COV (\%) & 12.5 & \\
\hline
\end{tabular}


Table 51. Room-temperature in-air uniaxial flexural properties of the 3-mm-thick choppedcarbon-fiber composite

\begin{tabular}{lcc}
\hline Specimen number & Maximum load (N) & MOR (MPa) \\
\hline Z6-1 & 396 & 292 \\
Z6-7 & 494 & 407 \\
Z6-13 & 449 & 359 \\
Z6-19 & 430 & 321 \\
Z6-20 & 411 & 331 \\
Z6-23 & 398 & 303 \\
Z6-29 & 371 & 278 \\
Z6-35 & 322 & 245 \\
& & \\
Average & 409 & 317 \\
SD & 51.6 & 50.1 \\
COV (\%) & 12.6 & 15.8 \\
\hline
\end{tabular}

Table 52. Room-temperature in-air uniaxial flexural properties of the 1.5-mm-thick chopped-carbon-fiber composite

\begin{tabular}{lcc}
\hline Specimen number & Maximum load (N) & MOR (MPa) \\
\hline ZZ3-1 & 121 & 309 \\
ZZ3-3 & 93.7 & 252 \\
ZZ3-5 & 125 & 293 \\
ZZ3-7 & 80.1 & 222 \\
ZZ3-9 & 119 & 340 \\
ZZ3-11 & 128 & 341 \\
& & \\
Average & 111 & 293 \\
SD & 19.5 & 47.6 \\
COV (\%) & 17.5 & 16.3 \\
\hline
\end{tabular}


Table 53. Uniaxial flexural properties of the 3-mm-thick chopped-carbon-fiber composite at different temperatures

\begin{tabular}{|c|c|c|}
\hline Specimen number & MOR (MPa) & Temperature factor \\
\hline & $-40^{\circ} \mathrm{C}$ & \\
\hline Z6-4 & 480 & 1.51 \\
\hline Z6-10 & 316 & 1.00 \\
\hline Z6-16 & 323 & 1.02 \\
\hline $\mathrm{Z} 6-26$ & 428 & 1.35 \\
\hline $\mathrm{Z} 6-32$ & 417 & 1.31 \\
\hline Z6-38 & 418 & 1.32 \\
\hline Average & 397 & 1.25 \\
\hline SD & 64.4 & \\
\hline \multirow[t]{2}{*}{$\operatorname{COV}(\%)$} & 16.3 & \\
\hline & $70^{\circ} \mathrm{C}$ & \\
\hline Z6-2 & 216 & 0.68 \\
\hline Z6-8 & 269 & 0.85 \\
\hline Z6-14 & 242 & 0.76 \\
\hline Z6-24 & 260 & 0.82 \\
\hline Z6-30 & 244 & 0.77 \\
\hline Z6-36 & 250 & 0.79 \\
\hline Average & 247 & 0.78 \\
\hline SD & 18.1 & \\
\hline \multirow[t]{2}{*}{$\operatorname{COV}(\%)$} & 7.35 & \\
\hline & $120^{\circ} \mathrm{C}$ & \\
\hline Z6-3 & 152 & 0.48 \\
\hline Z6-9 & 154 & 0.49 \\
\hline Z6-15 & 179 & 0.56 \\
\hline Z6-25 & 212 & 0.67 \\
\hline Z6-31 & 174 & 0.55 \\
\hline Z6-37 & 156 & 0.49 \\
\hline Average & 171 & 0.54 \\
\hline SD & 22.9 & \\
\hline $\operatorname{COV}(\%)$ & 13.4 & \\
\hline
\end{tabular}


Table 54. Effects of $1000-\mathrm{h}$ exposure in $23^{\circ} \mathrm{C}$ distilled water on uniaxial flexural properties of 3-mm-thick chopped-carbon-fiber composite

\begin{tabular}{lcc}
\hline Specimen number & MOR (MPa) & Strength factor \\
\hline Z6-5 & 273 & 0.86 \\
Z6-11 & 276 & 0.87 \\
Z6-17 & 292 & 0.92 \\
Z6-21 & 299 & 0.94 \\
Z6-27 & 336 & 1.06 \\
Z6-33 & 267 & 0.84 \\
& & \\
Average & 291 & 0.92 \\
SD & 25.5 & \\
COV (\%) & 8.76 & \\
\hline
\end{tabular}

Table 55. Effects of 100-h exposure in windshield washer fluid on uniaxial flexural properties of 3-mm-thick chopped-carbon-fiber composite

\begin{tabular}{lcc}
\hline Specimen number & MOR (MPa) & Strength factor \\
\hline Z6-6 & 373 & 1.18 \\
Z6-12 & 334 & 1.05 \\
Z6-18 & 320 & 1.01 \\
Z6-22 & 369 & 1.16 \\
Z6-28 & 275 & 0.87 \\
Z6-34 & 338 & 1.07 \\
& & \\
Average & 335 & 1.06 \\
SD & 35.9 & \\
COV (\%) & 10.7 & \\
\hline
\end{tabular}


Table 56. Biaxial flexural properties of the 3-mm-thick chopped-carbon-fiber composite at different temperatures

\begin{tabular}{lcc}
\hline Specimen number & Maximum load (N) & Temperature factor \\
\hline & $\mathbf{2 3}^{\circ} \mathbf{C}$ & \\
Z6-2 & 9644 & - \\
Z6-7 & 8665 & - \\
Z6-8 & 8990 & - \\
Z6-12 & 8131 & - \\
Z6-21 & 8723 & - \\
Z6-24 & 9688 & - \\
& & \\
Average & 8974 & - \\
SD & 605 & \\
COV (\%) & 6.74 & \\
& $\mathbf{1 2 0} \mathbf{C}$ & 0.68 \\
Z6-3 & 6090 & 0.56 \\
Z6-9 & 4995 & 0.56 \\
Z6-10 & 5066 & 0.54 \\
Z6-13 & 4871 & 0.55 \\
Z6-20 & 4960 & 0.68 \\
Z6-25 & 6107 & \\
& & 0.60 \\
Average & 5348 & \\
SD & 585 & \\
COV (\%) & 10.9 & \\
\hline
\end{tabular}

Table 57. Biaxial flexural properties of the 1.5-mm-thick chopped-carbon-fiber composite at different temperatures

\begin{tabular}{ccc}
\hline Specimen number & Maximum load (N) & Temperature factor \\
\hline & $\mathbf{2 3}^{\circ} \mathbf{C}$ & \\
ZZ3-1 & 3950 & - \\
ZZ3-3 & 3981 & - \\
ZZ3-5 & 4960 & - \\
& & - \\
Average & 4297 & \\
& $\mathbf{1 2 0}^{\circ} \mathbf{C}$ & 0.45 \\
ZZ3-2 & 1944 & 0.47 \\
ZZ3-4 & 2006 & 0.51 \\
ZZ3-6 & 2180 & \\
& & 0.48 \\
Average & 2043 & \\
\hline
\end{tabular}


Table 58. Effects of environment on biaxial flexural properties of the 3-mm-thick choppedcarbon-fiber composite

\begin{tabular}{lcc}
\hline Specimen number & Maximum load (N) & Knockdown factor \\
\hline \multicolumn{3}{c}{$\mathbf{1 0 0 0}^{\mathbf{h}}$ in $\mathbf{2 3}^{\circ} \mathbf{C}$ distilled water } \\
Z6-4 & 9266 & 1.03 \\
Z6-14 & 6757 & 0.75 \\
Z6-16 & 8367 & 0.93 \\
Z6-17 & 7433 & 0.83 \\
Z6-19 & 8167 & 0.91 \\
Z6-26 & 8661 & 0.97 \\
& & \\
Average & 8108 & 0.90 \\
SD & 894 & \\
COV (\%) & 11.0 & \\
& 8968 & 1.00 \\
Z6-5 & 8794 & 0.98 \\
Z6-11 & 8460 & 0.94 \\
Z6-15 & 10026 & 1.12 \\
Z6-18 & 9537 & 1.06 \\
Z6-22 & 9915 & 1.10 \\
Z6-27 & & \\
& 9283 & 1.03 \\
Average & 637 & \\
SD & 6.87 & \\
COV (\%) & &
\end{tabular}


ORNL/TM-2003/114

\section{INTERNAL DISTRIBUTION}

1. R. G. Boeman

2. J. G. Hansen

3. A. Ionita

4. E. Lara-Curzio

5-14 D. J. Naus

15. R. E. Norris

16. M. H. Rawlins
17. M. B. Ruggles-Wrenn

18. P. A. Sklad

19. C. D. Warren

20-21. Y. J. Weitsman

22. R. E. Ziegler

23. ORNL Laboratory Records-RC

\section{EXTERNAL DISTRIBUTION}

24. M. Abdallah, Hexcel Carbon Fibers, Research and Technology, P.O. Box 18748, Salt Lake City, UT 84118-0748

25. M. M. Fisher, American Plastics Council, 1300 Wilson Boulevard, Suite 800, Arlington, VA 22209

26. R. B. Freeman, The Budd Company, 1515 Atlantic Boulevard, Auburn Hills, MI 48326

27. R. Gjerde, Textron Automotive Company, 100 Brady Road, Americus, GA 31709

28-57. L. Berger, Automotive Composite Consortium, General Motors, 30500 Mound Road, Mail Code 480-106-710, Box 9055, Warren, MI 48090-9055

58. J. M. Henshaw, Department of Mechanical Engineering, The University of Tulsa, 600 S. College Avenue, Tulsa, OK 74104-3189

59. G. A. Holmes, National Institute of Standards and Technology, Bldg. 224, Room B116, Mail Stop: Room B108, Gaithersburg, MD 20899

60. D. Kossak, Cambridge Industries, 29333 Stephenson Highway, Madison Heights, MI 48071

61. F. G. Krautz, Vetrotex Certainteed, 4515 Allendale Road, Wichita Falls, TX 763102199

62. G. R. Miesel, Ashland Chemical Company, 900 Wilshire Drive, Suite 100, Troy, MI 48084

63. E. Ostwald, Dow Automotive, 1600 Harmon Road, Auburn Hills, MI 48326

64. S. R. Reeve, National Composite Center, 2000 Composite Drive, Kettering, OH 45420

65. C. R. Schultheisz, National Institute of Standards and Technology, Building 224, Room A209, Gaithersburg, MD 20899

66. T. D. Seagrave, Bayer Corporation, 100 Bayer Road, Pittsburgh, PA 15205

67. D. R. Secrist, Fortafil Fibers, Inc., 121 Cardiff Valley Road, Rockwood, TN 37854

68. D. Stewart, Stewart Automotive Research, LLC, 1260 Shotwell Street, Houston, TX 77020

69. S. J. Winckler, Cyclics Corporation, 747 Pierce Road, Clifton Park, NY 12065

70-73. J. A. Carpenter, U.S. Department of Energy, 1000 Independence Avenue, SW, Washington, DC 20585

74. S. Diamond, U.S. Department of Energy, 1000 Independence Avenue, SW, Washington, DC 20585 